University of Louisville ThinkIR: The University of Louisville's Institutional Repository

Electronic Theses and Dissertations

$8-2019$

\title{
Formally designing and implementing cyber security mechanisms in industrial control networks.
}

Mehdi Sabraoui

University of Louisville

Follow this and additional works at: https://ir.library.louisville.edu/etd

Part of the Computer and Systems Architecture Commons, Controls and Control Theory Commons, Information Security Commons, OS and Networks Commons, Other Computer Engineering Commons, Software Engineering Commons, Systems Architecture Commons, and the Theory and Algorithms Commons

\section{Recommended Citation}

Sabraoui, Mehdi, "Formally designing and implementing cyber security mechanisms in industrial control networks." (2019). Electronic Theses and Dissertations. Paper 3271.

https://doi.org/10.18297/etd/3271

This Doctoral Dissertation is brought to you for free and open access by ThinkIR: The University of Louisville's Institutional Repository. It has been accepted for inclusion in Electronic Theses and Dissertations by an authorized administrator of ThinkIR: The University of Louisville's Institutional Repository. This title appears here courtesy of the author, who has retained all other copyrights. For more information, please contact thinkir@louisville.edu. 


\title{
FORMALLY DESIGNING AND IMPLEMENTING CYBER SECURITY MECHANISMS IN INDUSTRIAL CONTROL NETWORKS
}

\author{
BY \\ Mehdi Sabraoui \\ B.S., University of Louisville, 2013 \\ M.Eng., University of Louisville, 2014 \\ A Dissertation \\ Submitted to the Faculty of the \\ J. B. Speed School of Engineering \\ in Partial Fulfillment of the Requirements \\ for the Degree of \\ Doctor of Philosophy \\ in Computer Science and Engineering \\ Department of Computer Science and Engineering \\ J.B Speed School of Engineering \\ University of Louisville \\ Louisville, Kentucky
}

August 2019 



\title{
FORMALLY DESIGNING AND IMPLEMENTING CYBER SECURITY MECHANISMS IN INDUSTRIAL CONTROL NETWORKS
}

\author{
BY \\ Mehdi Sabraoui \\ B.S., University of Louisville, 2013 \\ M.Eng., University of Louisville, 2014 \\ A Dissertation Approved on
}

July 23, 2019

By the following Dissertation Committee members

Dr. Adrian P. Lauf, Dissertation Director

Dr. Jeffrey L. Hieb, Dissertation Co-Director

Dr. Roman V. Yampolskiy

Dr. Michael Losavio

Dr. Adel Elmaghraby 


\section{DEDICATION}

This dissertation is dedicated to my parents

Rebecca Sabraoui and Ben Sabraoui

whose hard work and constant support have granted me this privilege. 


\section{ACKNOWLEDGEMENTS}

I cannot understate how much the support of my advisors, Dr. Jeff Hieb and Dr. Adrian Lauf, have helped me through the academic, professional, and emotional ups and downs of this journey. I would also like to extend my deepest gratitude to Dr. Adel Elmaghraby for his clever administrative support for me and all the students in the department who come to him for help and guidance. I am grateful to the rest of my committee members, Dr. Roman Yampolskiy and Dr. Michael Losavio, for their invaluable insights and friendly conversations through the tough times. Finally, I want to recognize all the friends and family who have been patient and understanding with my occasional absences through the past few years. 


\begin{abstract}
FORMALLY DESIGNING AND IMPLEMENTING CYBER SECURITY MECHANISMS IN INDUSTRIAL CONTROL NETWORKS
\end{abstract}

Mehdi Sabraoui

July 23, 2019

This dissertation describes progress in the state-of-the-art for developing and deploying formally verified cyber-resilient devices in industrial control networks. It begins by detailing the unique struggles that are faced in industrial control networks and why concepts and technologies developed for securing traditional networks might not be appropriate. It uses these unique struggles and examples of contemporary cyber-attacks targeting control systems to argue that progress in securing control systems is best met with formal verification of systems, their specifications, and their security properties. This dissertation then presents a development process and identifies two technologies, TLA+ and seL4, that can be leveraged to produce a high-assurance embedded security device.

The method presented in this dissertation takes an informal design of an embedded device that might be found in a control system and 1) formalizes the design within TLA+, 2) creates and mechanically checks a model built from the formal design, and 3) translates the TLA+ design into a component-based architecture of a native seL4 
application. The later chapters of this dissertation describe an application of the process to a security preprocessor embedded device that was designed to add security mechanisms to the network communication of an existing control system. The device and its security properties are formally specified in TLA+ in chapter 4, mechanically checked in chapter 5, and finally its native seL4 architecture is implemented in chapter 6 . Finally, the conclusions derived from the research are laid out, as well as some possibilities for expanding the presented method in the future. 


\section{TABLE OF CONTENTS}

PAGE

DEDICATION .................................................................................................. ii

ACKNOWLEDGEMENTS ................................................................................ iv

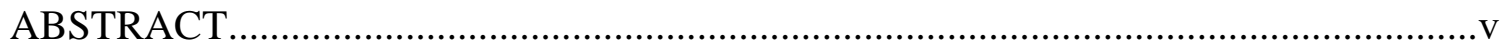

LIST OF TABLES ...........................................................................................

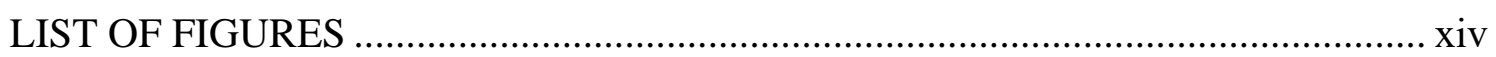

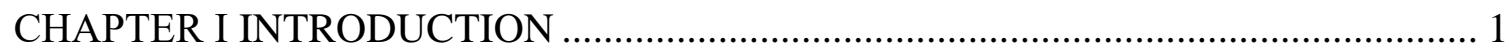

1.1 Industrial Control Systems ................................................................... 3

1.2 Components of Industrial Control ......................................................... 7

1.3 ICS Network Devices and Requirements ............................................... 8

1.4 Cyber Security for Industrial Control Systems ....................................... 11

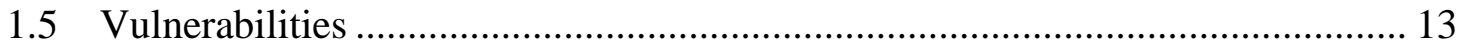

1.6 ICS Policies \& Best Practices ............................................................ 17

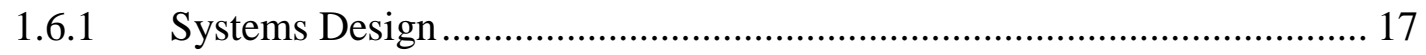

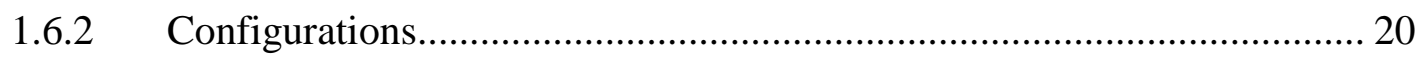

1.6.3 Patch Management and Disaster Recovery....................................... 23

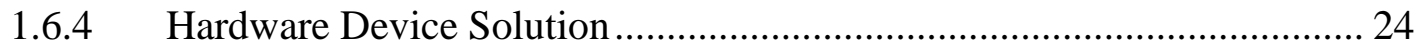

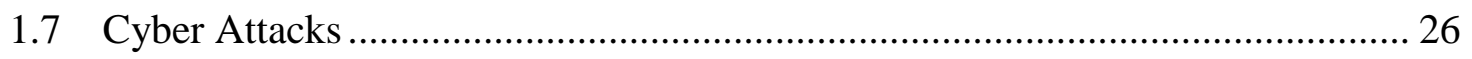

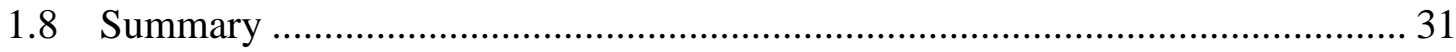

CHAPTER II LITERATURE SURVEY .................................................................... 32

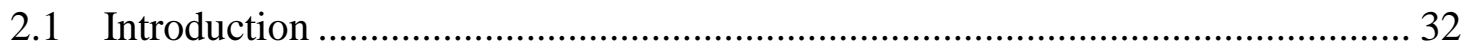

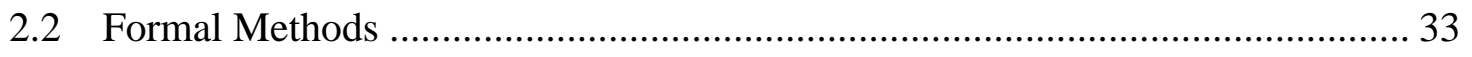


2.2.1 Model Checking .................................................................................... 34

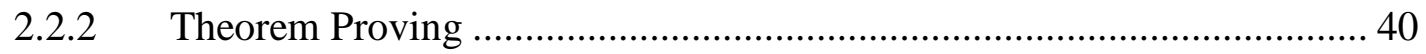

2.2.3 Standards and Certifications …………………...................................... 40

2.2.4 Limits of Formal Methods ...................................................................... 43

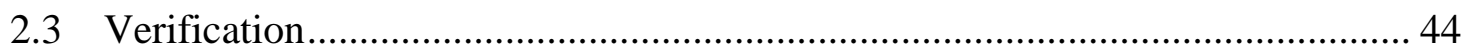

2.3.1 Verification of a Cryptographic Primitive: SHA-256................................. 45

2.3.2 Verified correctness and security of OpenSSL HMAC ............................. 46

2.3.3 HACL*: A Verified Modern Cryptographic Library.................................. 48

2.3.4 Breaking and fixing the Needham-Schroeder Public-Key Protocol using

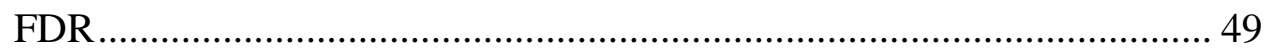

2.3.5 Implementing TLS with Verified Cryptographic Security ........................ 51

2.3.6 The Temporal Logic of Actions, TLA+................................................ 53

2.3.7 Use of Formal Methods at Amazon Web Services .................................... 54

2.4 Modeling and Verification of Operating Systems.............................................. 55

2.4.1 The Bell-La Padula model ...................................................................... 56

2.4.2 The transfer of information and authority in a protection system ............. 58

2.4.3 seL4: formal verification of an OS kernel ............................................... 60

2.4.4 The HACMS program: using formal methods to eliminate exploitable

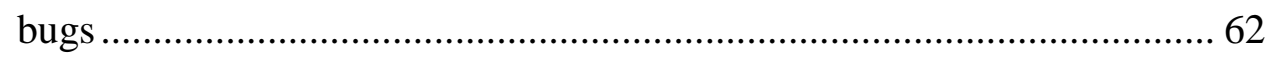

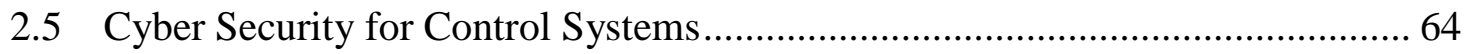

2.5.1 Formal Vulnerability Analysis of a Security System for Remote Fieldbus

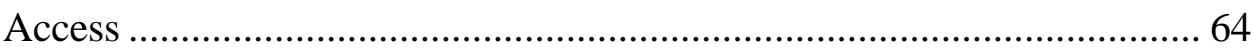

2.5.2 Towards Formal Security Analysis of Industrial Control Systems .......... 66 
2.5.3 Anomaly detection in cyber-physical systems: A formal methods approach 67

2.5.4 Formal modelling and analysis of DNP3 secure authentication ............... 68

2.5.5 Attack taxonomies for the Modbus protocols ................................... 70

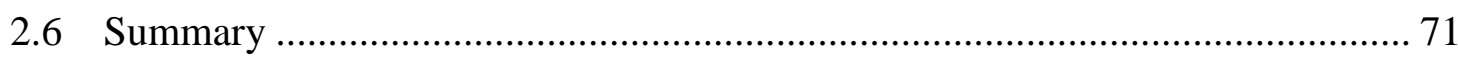

CHAPTER III HIGH ASSURANCE CYBER-SECURITY DEVICES FOR INDUSTRIAL CONTROL SYSTEMS USING TLA+ AND SEL4 ............................................ 72

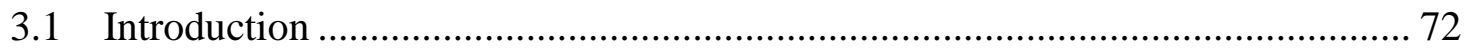

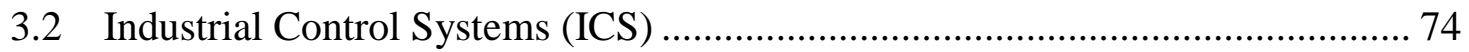

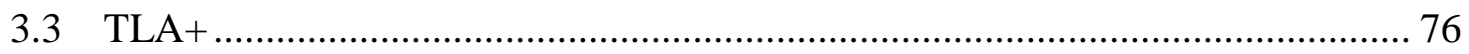

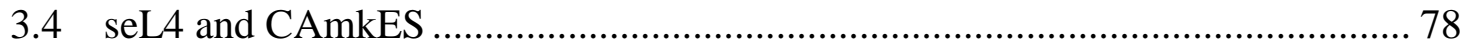

3.5 Application of Verified Systems for Control Systems Security....................... 80

3.6 Translation of TLA+ and PlusCal into CAmkES ........................................ 82

3.7 Security Preprocessor as Previously Designed ........................................... 86

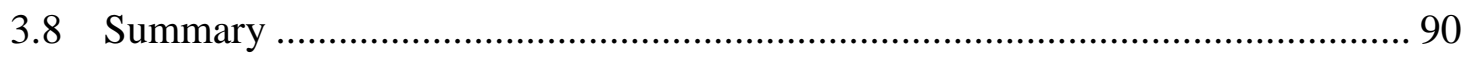

CHAPTER IV MODELING A BUMP-IN-THE-WIRE SECURITY

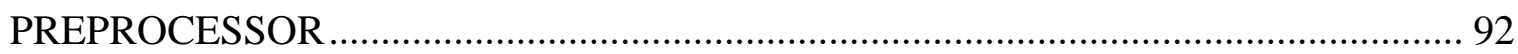

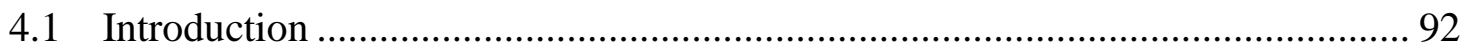

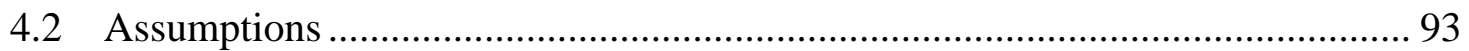

4.3 Formal TLA+ Specifications for Components and Properties ......................... 95

4.3.1 Modeling the Trusted Network Component ..................................... 95

4.3.2 Modeling the Untrusted Network Component.................................... 104

4.3.3 Modeling the Protocol Checking Component..................................... 110

4.3.4 Modeling the Cryptographic Component ....................................... 117 
4.3.5 Modeling the System ........................................................................ 123

4.3.6 Additional Operators and Functions in TLA+ ..................................... 126

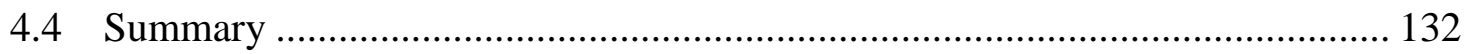

CHAPTER V MODEL CHECKING, INPUT VALUES, STATES .............................. 133

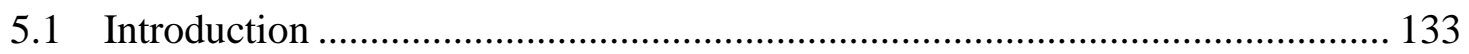

5.2 TLC Model Checker.................................................................................... 133

5.3 State Explosion Considerations ..................................................................... 135

5.4 Trusted Network Component States and Inputs ............................................ 138

5.5 Untrusted Network Component States and Inputs ............................................. 140

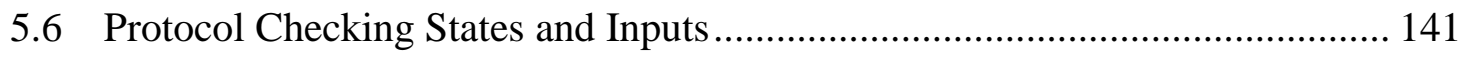

5.7 Cryptographic Component States and Inputs .................................................... 141

5.8 System Model States and Inputs ……………….......................................... 142

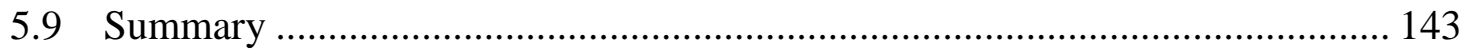

CHAPTER VI CAMKES ARCHITECTURE FOR A BUMP-IN-THE-WIRE SECURITY

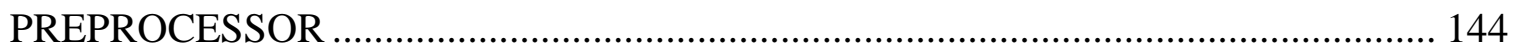

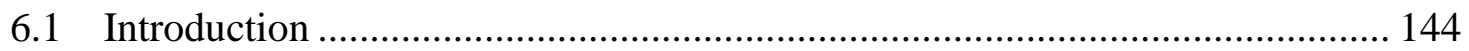

6.2 CAmkES Definitions for Components, Interfaces, and Connections ............... 145

6.2.1 Modtx: The Trusted Network Interface ……………........................... 146

6.2.2 Signtx: The Untrusted Network Interface............................................ 148

6.2.3 Modchk: The Protocol Checker .............................................................. 152

6.2.4 Crypto: The Cryptographic Service …………….................................... 154

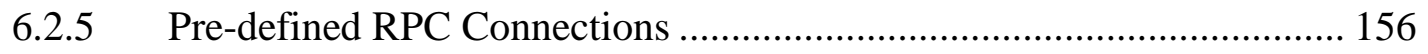

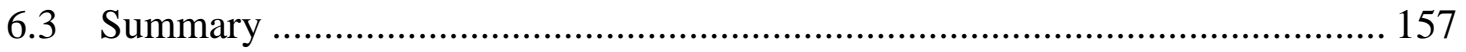


CHAPTER VII CONCLUSIONS AND FUTURE WORK.

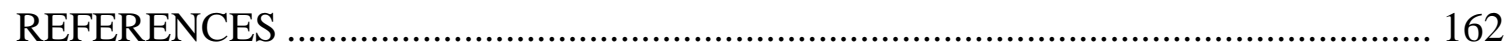

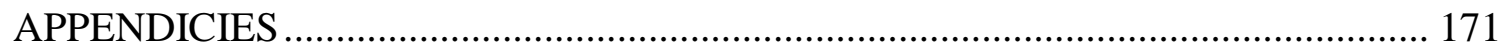

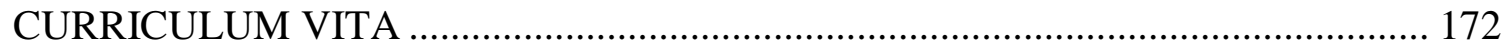




\section{LIST OF TABLES}

TABLE

PAGE

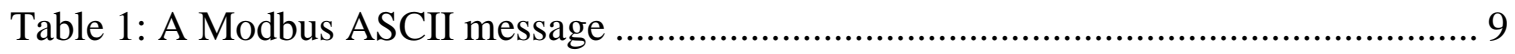

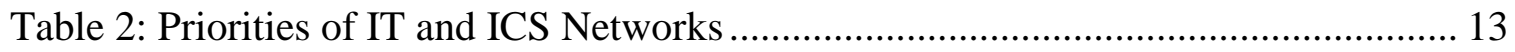

Table 3: Stuxnet Capabilities and Targeted Vulnerability ......................................... 28

Table 4: EAL descriptions and example products ................................................... 41

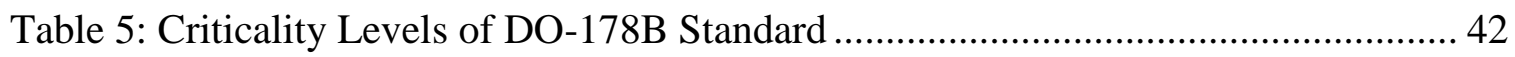

Table 6: Benefits of TLA+ in Amazon's Ecosystem. Adapted from [103] ..................... 55

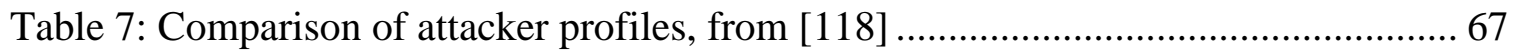

Table 8: Sample of Possible Attacks against Modbus .............................................. 70

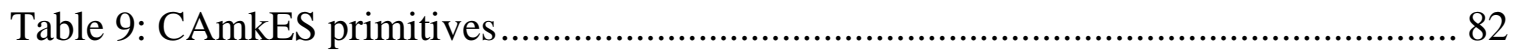

Table 9: The desired properties of the Trustnet_in thread ........................................ 98

Table 10: TLA+ symbols used in the property definitions for trustnet_out .................... 99

Table 11: The desired properties of the Trustnet_out thread .................................... 101

Table 12: Properties of the Untrustnet_in thread ...................................................... 106

Table 13: The desired properties of the Unrustnet_out thread .................................. 109

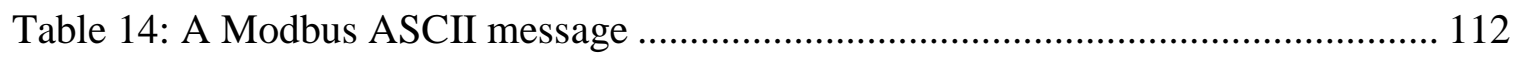

Table 15: Modbus function codes. Adapted from [123] ......................................... 113

Table 16: The desired properties of the Modchk thread .......................................... 115

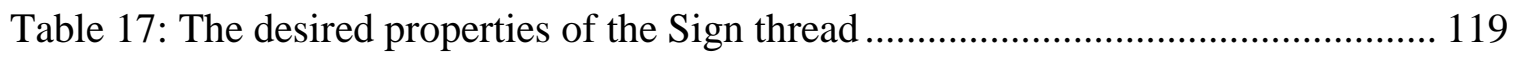


Table 18: Desired properties of the Verify thread

Table 19: Desired informal properties of the security preprocessor.

Table 20: The desired formal properties of the security preprocessor. 125

Table 21: TLC Running Statistics for the trusted network component 139

Table 22: Test messages for Trustnet_in 139

Table 23: TLC Running Statistics for the untrusted network component 141

Table 24: TLC Running Statistics for the protocol checking component 141

Table 25: TLC Running Statistics for the cryptographic component 142

Table 26: TLC Running Statistics for the security preprocessor 142

Table 27: Relationship between TLA+ specifications and CAmkES components 146

Table 28: A Modbus ASCII message 153 


\section{LIST OF FIGURES}

\section{FIGURE}

PAGE

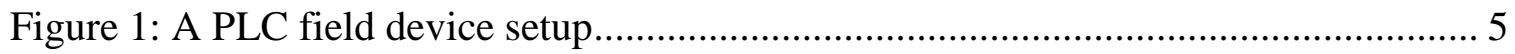

Figure 2: A PLC process network setup .......................................................... 6

Figure 3: Proposed network design for a unidirectional gateway. Taken from [49] ........ 26

Figure 4: An example Kripke structure. Taken from [69] ........................................ 36

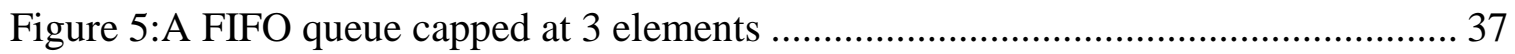

Figure 6: Range of costs required for completing product evaluations at various

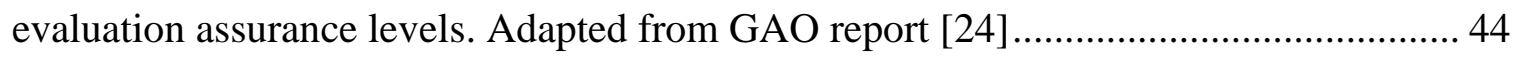

Figure 7: Range of sample cost of NIAP evaluations to vendors by evaluation assurance

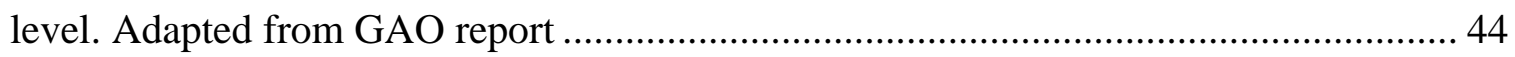

Figure 8: The verified components of the TLS1.3 suite. Image from https://project-

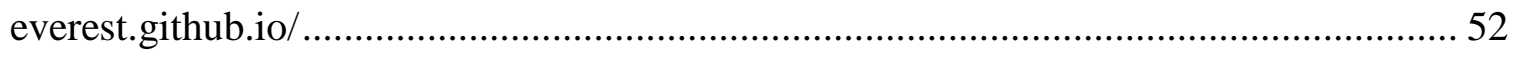

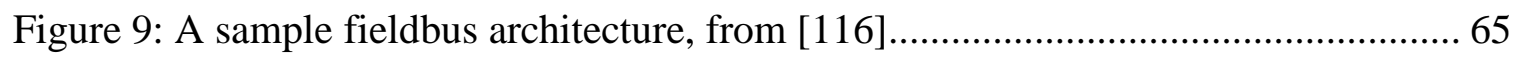

Figure 10: Development steps for verifying embedded control system devices. ............ 74

Figure 11: A typical ICS network topology, adapted from [130] ................................ 75

Figure 12: Development steps for verifying seL4 designs using TLA+...................... 81

Figure 13: CAmkES directory setup for an example application. Each component has its own directory housed within the "Components" directory. Each component directory has a camkes file and a src folder containing $\mathrm{C}$ code 83 
Figure 14: PlusCal definitions for Send and Receive macros. 84

Figure 15: Send macros in TLA+ and their translations into CAmkES connections. Declarations of seL4RPCCall connections from the Modtx component to the protocol

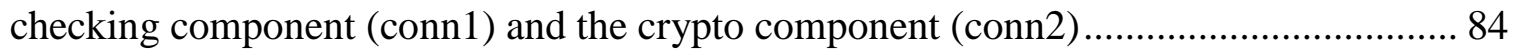

Figure 16: A Send macro in TLA+ and its translation to a CAmkES interface ............. 85

Figure 17: Translation of a PlusCal send macro to a CAmkES component definition .... 85

Figure 18: Connection from the control center (left) to RTU (right) on a typical SCADA

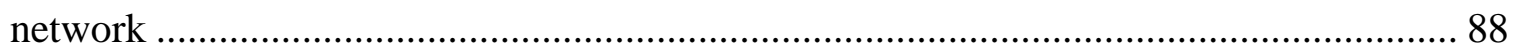

Figure 19: Connection from the control center to RTU with the FD-SPP installed ......... 88

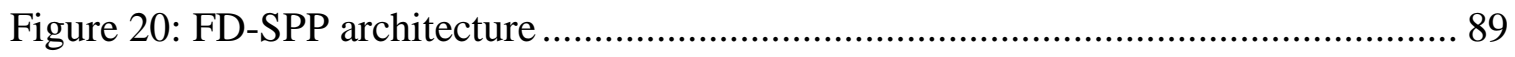

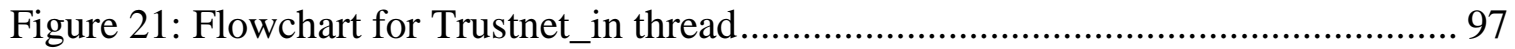

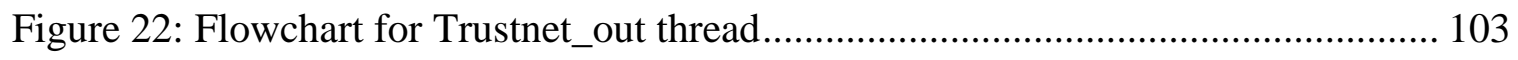

Figure 23: Flowchart for untrustnet_in thread ................................................... 105

Figure 24: Flowchart for Untrustnet_out thread ................................................... 108

Figure 25: Flowchart for the Modchk component .................................................. 114

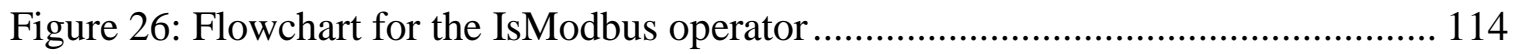

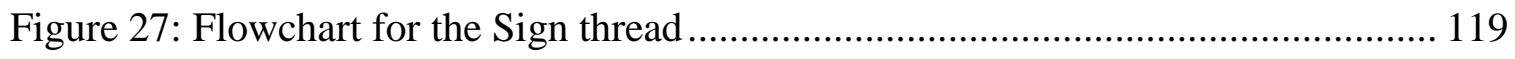

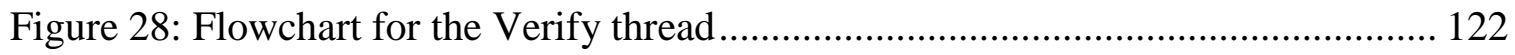

Figure 29: The state space generated from (13) .................................................. 136

Figure 30: The state space generated from Equation 14........................................ 137

Figure 31: Development steps for verifying seL4 designs using TLA+_.................... 145

Figure 32: CAmkES output for the system architecture ........................................ 146

Figure 33: Flow of messages through Modtx ................................................... 148 
Figure 34: The Modtx component definition....................................................... 149

Figure 35: The ModtxIface interface definition...................................................... 149

Figure 36: Flow of messages through Signtx ...................................................... 151

Figure 37: The Signtx component definition .................................................... 152

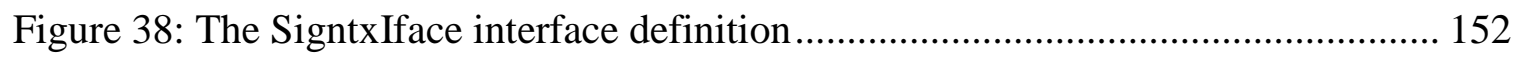

Figure 39: The Modchk component definition ....................................................... 154

Figure 40: The ModchkIface interface definition ................................................. 154

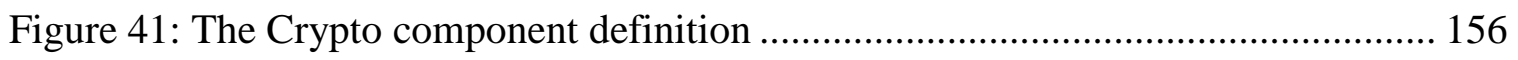

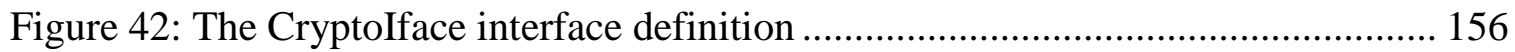

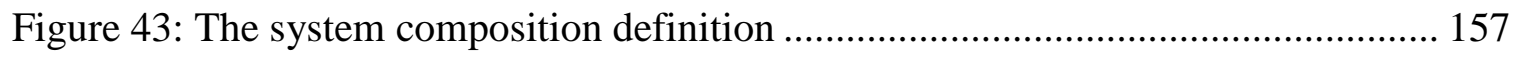




\section{CHAPTER I}

\section{INTRODUCTION}

Industrial control system (ICS) is a general term describing multiple configurations of networked industrial computer systems [1]. ICSs regulate factory floors and utilities such as power grids, dams, water-treatment facilities, and many more. Unlike typical corporate IT networks, ICS engineers value availability above confidentiality [2]. Keeping the data in the system private is not as important as keeping the system running. Threats to an ICS reflect this priority: an attacker seeks to disturb and disrupt the controlled process. Disrupting these processes could lead to physical consequences affecting the surrounding area like the attack on Maroochy Water Services, a watertreatment plant in Australia. A disgruntled employee manipulated the control systems to seize control from plant engineers and dump sewage into surrounding parks and rivers [3]. The importance of availability disincentivizes ICS engineers making regular changes or updates to the systems for fear of unscheduled downtime.

A variety of factors have led to the current challenge-riddled state of ICS cyber security. One of the primary reasons for lack of security is that ICS networks have historically been physically isolated from the greater internet [4][5]. SCADA communications protocols were therefore designed to prevent accidental corruption from a well-meaning operator rather than a purposeful attack. Incidents like Stuxnet have shown that air-gapping a SCADA network is no longer enough protection [6]. Many 
industry-standard protocols such as MODBUS, EtherNet/IP, Profibus, and others have no means for ensuring the validity of messages [7], [8], [5]. This presents an opening for a malicious user to pretend to be either an operator controlling a Programmable Logic Controller (PLC) directly or an intermediate PLC controlling a device located at a remote substation. Once an attacker is inside a network any message the attacker sends is trusted and processed by the devices --- to potentially disastrous effects. A layering of multiple defensive strategies is required to mitigate this vulnerability and adding security to the communication protocols can cover some of the security holes. The need for security in protocols is shown in DNP3's efforts to create Secure Authentication (SA) within the DNP3 specification. The expanded capability of DNP3 SA currently offers protection against many common attacks by adding a challenge-response system for ensuring validity of communication across the DNP3 network[9]. DNP3 Secure Authentication is limited in its coverage of security concerns: it applies only to infrastructure currently using DNP3 and can be troublesome on networks using a variety of networking technologies to connect central control facilities to remote substations.

Formal methods are techniques for adding a high level of assurance to designs and implementations[10]-[14]. Human languages are ambiguous by nature and thus are not suited to describing software beyond the planning phases. Formal modeling can be used not only to create explicit designs, but also to logically prove certain properties of the designs. Proofs of security and fail-safety can be very useful in an ICS environment. This paper looks to use formal modeling and logic to prove security and assurance properties of a protocol designed to encapsulate SCADA traffic. 
This chapter seeks to provide the reader with enough background information on the fields of Industrial Control Systems (ICS), ICS security, and formal methods to understand the context behind the research presented in the following chapters. The practical aspects of this research require a mix of resources from peer-reviewed academic papers, established industry standards, and white papers.

\subsection{Industrial Control Systems}

Industrial control extends it reach across electrical grids, wastewater treatment facilities, dams, water distribution systems, agricultural irrigation systems, pipelines for oil and natural gas, railroads, manufacturing plants, and air traffic control. The physical processes in these systems are controlled using electrical, mechanical, hydraulic, or pneumatic components [1]. Historically, such processes were operated by humans using analog mechanisms. Advances in digital technology offered new opportunities for control systems as integrated circuits and microprocessors started to replace old analog control loops and their human operators. As more of the controls became digital, the value of an interconnected control system became apparent. New communication mediums and protocols were developed to extend the reach of the system to geographically distant substations such as a neighborhood water tower located miles away from the city's central distribution facility.

While advancements in ICS technology sometimes mirror that of a traditional corporate network, its requirements and operation do not. ICS networks are seeing more use of Ethernet, however, the protocols selected allow for some level of determinism, real-time collection, and low overhead [15]. Traditional networks are shallow in their functionality with a very limited set of protocols and standards. ICS networks are more 
varied with separate entities performing separate duties with physical goals in mind [16]. Knowledge of a traditional IT network will help in understanding an ICS network, but some key terminology explained below helps illustrate the difference.

Supervisory Control and Data Acquisition (SCADA) systems are used to control and monitor physical systems spread over a wide geographical region [15], [17], [18]. The first SCADA systems were simple configurations of sensors connected to dials, lights analog strip charts organized on a panel. Changes in the physical system would be picked up by the sensor and turn a dial or register on the chart in real time. A human would read the panel then act to adjust the system as needed. This basic system, while admirably fulfilling its purpose of getting the operator information about the system in real time, had some key shortcomings: an operator had to be present and monitoring the system at all times, each output on the panel was directly connected to a sensor so wiring new sensors became unwieldy, everything was local - substations could not be monitored from a central location, reconfiguring the system became increasingly difficult as the system grew, the type of data that could be collected and displayed was basic, and storage of the data was virtually non-existent.

Modern SCADA systems utilize advancements in communication to operate over distances of a few hundred yards to thousands of miles. Modern visual displays and microcontrollers/microcomputers allows more flexibility in the data collected and the control that can be exerted upon the system. There are three configurations for modern SCADA systems: open loop where the controls on the system are defined in advance and the state of the system has no bearing on the automated instructions, closed loop where the data acquired from the physical system is fed into the control modules and 
instructions are adjusted accordingly, and manual systems in which a human manually controls the system based on the data collected.

Distributed control systems (DCS) are used to control the automation of industrial processes at a single location. DCS oversee multiple subsystems that each have separate responsibilities at individual points in a process. Through a DCS these subsystems can be integrated with feedback and/or feed forward loops to compensate for variability at each stage of the process. This allows the process as a whole to self-correct in the event a single point misbehaves. DCS are widespread in oil refineries and food, chemical, and automotive production plants. These connect with programmable logic controllers (PLCs) that governs industrial equipment and processes. PLCs can be used as field devices on SCADA and DCS systems as seen in Figure 1 or as primary control devices in smaller systems like in Figure 2.

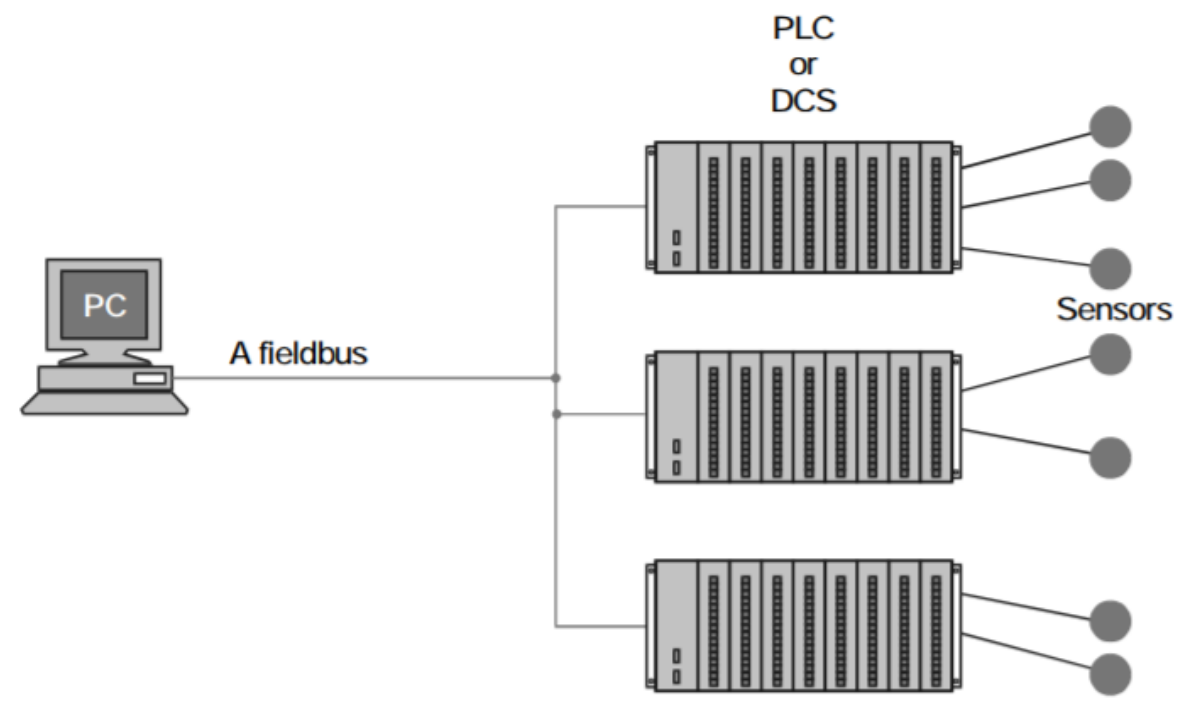

Figure 1: A PLC field device setup 


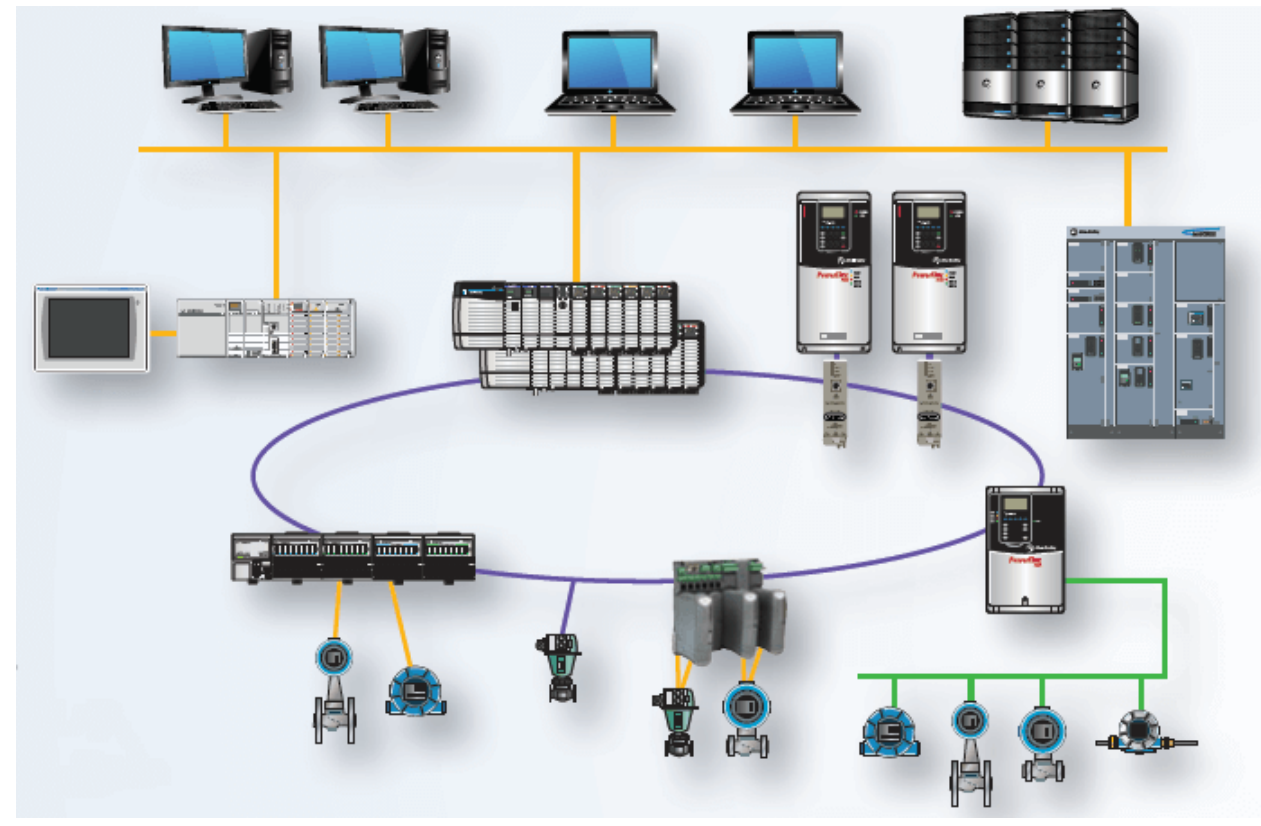

Figure 2: A PLC process network setup

Understanding the manufacturing needs of the industries that use ICS helps to understand the ICS configurations. There are manufacturing industries and distribution industries [16]. Manufacturing industries typically involve a single location such as a factory and are further split into continuous manufacturing process wherein the process from raw materials to finished product runs continuously and batch manufacturing process where the process is broken into distinct steps producing a specific amount of the product. Examples of continuous manufacturing processes include petroleum and distillation in a chemical plant. Food and consumer goods are examples of batch manufacturing. The small area of operation allows for greater reliability and performance in the networking technology used within the factory. Distribution industries, on the other hand, control devices spread over large distances such as oil and water pipelines and railway systems and offer less assurance in communication. These systems use leased lines, radio frequency, and satellite links [15] to overcome these great distances, each of 
these with their own security and reliability concerns. Distribution industries are typically designed to handle the reliability and timing challenges that come with long distance communications.

\subsection{Components of Industrial Control}

The differences between IT networks and control networks can further be detailed in the devices and protocols that make up the environment. A Remote Terminal Unit (RTU) interface with machinery and sensors in modern ICS networks. RTUs govern industrial equipment and processes. They are lower cost and lower capability than a PLC and are used in remote stations where less functionality is required and less user interaction is desired. RTUs often comes equipped with radio capabilities for wireless communications to the central stations. A control server (or master server) hosts the control software and sends instructions to the PLCs and RTUs around the network. This is usually located at the central control facility and is used to collect and process information relayed from field devices. An Intelligent Electronic Device (IED) is a "smart" sensor/actuator that may sit between the RTU and the machinery or it may replace the RTU entirely and communicate directly with the control server. IEDs have functionality to run simple routines to react to changes in the parameters of the system, but are usually polled by RTUs or PLCs and given instructions from there.

ICS operators manipulate the system through a Human Machine Interface (HMI). An HMI is software that allows the control engineers to monitor the elements of the processes under control. A typical HMI can allow an engineer to set alarms in case certain limits are exceeded, modify the processes, take manual control in case of emergency, and read reports on runtime information. HMIs can be located in the control 
center, on engineer workstations or laptops, and more recently on mobile devices. The data that is collected or calculated and any triggered events or alarms are usually saved and stored for later analysis. This data can be collected in a data historian that can reside on the control network or an outside network with security restrictions in place to prevent it from communicating in any way with the control network other than receiving information. A slave historian can be used to duplicate the historian's data onto a server on the corporate network for the business to access and analyze.

\subsection{ICS Network Devices and Requirements}

ICS networking concepts and requirements evolved from a need to rein in the wiring of early control systems. As described earlier, each sensor used to be wired directly to the meter displaying its reading to the operator. Each sensor required a separate wire for each binary digit it was expected to record [19]. This method of wiring was quickly outgrown and the industry requested a solution from its vendors and university researchers. The solution was a Fieldbus, a network that connected devices in the field such as RTUs and IEDs to the central facilities. Fieldbus is a broad term to describe all the communication technologies that solve this wiring problem. Many protocols, such as Modbus[20]-[22], Distributed Network Protocol (DNP3)[23][24][26], and Common Industrial Protocol (CIP) family of protocols [27], [28] are used on a fieldbus network. These protocols are responsible for handling any device identification in place of individual wires for each sensor. The medium for communication is undefined for a fieldbus and may include multiple technologies such as Ethernet, serial, satellite link, telephone lines, or radio frequency [15]. To this end, a modem is a device that can translate a digital signal into analog for easier transmission over any number of long- 
distance mediums. One modem would be connected at a remote substation to translate digital signals from the RTU into analog and another would be located at the central facility to translate the analog signal back into digital for use.

This research makes heavy use of Modbus, so it may benefit the reader to have a longer explanation of Modbus in particular. Modbus is an open communication protocol developed in 1979 by Modicon for use in ICS networks. ICS are difficult to install and difficult to upgrade and have longer lifecycles relative to corporate networks. This lead to operators preferring open standards and the proliferation of protocols like Modbus [16]. As described in the specification guide in [29], the base Modbus is a simple, stateless, call-and-response protocol. It contains a simple addressing scheme allowing for up to 247 devices on a common bus, a field for a function code that tells the target devices which procedure to run, and a data field that can contain up to 252 bytes for the target device to act on. There are two versions of Modbus: Modbus RTU and Modbus ASCII. Modbus RTU transmits raw bytes and uses a specific minimum time between bytes sent over the bus to distinguish between frames and a Cyclic Redundancy Check (CRC) to detect errors in transmission. Modbus ASCII operates on ASCII-encoded messages, utilizing two bytes where Modbus RTU would only require one. Modbus ASCII distinguishes frames with a colon. Whenever a device receives a ":" it knows a new message has started, regardless of where the previous message left off. To detect transmission errors, Modbus ASCII makes use of a Longitudinal Redundancy Check (LRC). The structure of a Modbus ACSII message is presented in Table 1. This research uses Modbus ASCII for simplicity.

Table 1: A Modbus ASCII message 


\begin{tabular}{|c|c|c|c|c|c|c|}
\hline Start & Address & $\begin{array}{c}\text { Function } \\
\text { Code }\end{array}$ & Sub code & Data & LRC & End \\
\hline “:” & 2 bytes & 2 bytes & $\begin{array}{c}2 \text { bytes } \\
\text { (optional) }\end{array}$ & $\begin{array}{l}\text { Up to } 504 \\
\text { bytes }\end{array}$ & 2 bytes & "Irln" \\
\hline
\end{tabular}

The growing interconnectedness of ICS networks with corporate networks and devices have led to incorporation of corporate network technologies. A router is a networking device that allows communication between logically separated networks. These are used to allow access to the control network from the corporate network and vice-versa. A firewall allows a network engineer to closely regulate the connections that are made across networks. A firewall (sometimes multiple [30], [31]) located at strategic points such as between the ICS and the corporate network or between the engineers terminal and the fieldbus can block unwanted network traffic from reaching the ICS. A remote access point is a device that allows control over the ICS remotely. Such devices include laptops, tablets, and smartphones that access the control network from anywhere through a Virtual Private Network (VPN), which encrypts traffic and "tunnels" through a public network.

Special considerations must be made when designing an ICS network. Each system is unique in its requirements and goals, and these factors inform the decisions made in selecting technologies and topologies. Depending on the nature of the industry, the timing requirements may range from 250 microseconds to 10 milliseconds. A response time that is less than the sensor's sample time is recommended [16]. This can necessitate processing power at a remote substation, as performing computations remotely might incur too significant of a delay. A distribution industry such as an oil pipeline would have SCADA components spread over thousands of miles with different 
options for communications at different substations. The complexity of control needed for the system might allow simple controllers with predefined routines or might require high-level decision making from a human operator such as in air traffic control [1]. The need for high uptime, $99.999 \%$ or 5 minutes and 35 seconds per year of allowable downtime per year in some cases [32], and reliability would push for a system with more redundancy and alternate forms of communication should one fail. To go along with availability, the impact of a failure in the system must be considered. A failure in a nuclear reactor could have significant environmental impacts and would require both redundant control systems and physical safety mechanisms. Finally, operator safety must be considered. A control network in a car must be able to detect a sudden application of the brakes to tighten the seatbelt, apply the automatic braking system, and deploy airbags if needed.

\subsection{Cyber Security for Industrial Control Systems}

Industrial Control Systems (ICS) regulate processes that, if compromised, can have a physical effect on the environment around them. A broken ICS process can cause damages to the facilities containing the machinery and/or endanger human life [33]. As with the design considerations varying across industry, so too do the means by which an attacker can cause harm. Strict timing requirements mean that slowing down response time would disrupt the system. This is especially true of close-loop systems, where a transmission time exceeds the sample time. This error can propagate and amplify over cycles to force the system into an unstable state [34], [35]. Programmable Logic Controllers (PLCs), Remote Terminal Units (RTUs) and Intelligent Electronic Devices (IEDs) are designed to be programmed and reprogrammed as needed to suit changing 
requirements in the system. An attacker could reprogram one of these devices to modify its behavior or adjust thresholds to effectively disable alarms. An attacker could modify or fake information being sent to PLCs and Human-Machine Interfaces (HMIs) to disguise unauthorized changes in the system or cause the operator to initiate inappropriate actions. As with a corporate or home network, malware-infected workstations can have degraded system performance or actively disrupt the system by modifying configurations. An attacker can also interfere with the safety mechanisms such as emergency shutdown systems, safety shutdown systems, or safety interlock systems [1], [36].

The design of ICS networks makes manipulating SCADA components simple. ICS networks were originally isolated from corporate networks and the greater networks, thus network traffic moving across the lines is inherently trusted. Early systems used specialized software and hardware with proprietary protocols. Modern systems are using cheap commercial off the shelf (COTS) hardware with open protocols and IT design principles that promote connectivity with corporate networks and erode the isolation that control networks used to enjoy [1][5]. While this integration of IT technology allows corporate network security measures to be utilized, the special considerations discussed in the previous section can limit their viability. These special considerations can also require new technologies to be developed.

When considering the CIA triad [37], the priorities for an ICS are different than traditional IT as seen in Table 2 [2]. Confidentiality is paramount for most IT systems. Trade secrets, banking information, employee personal information, and other sensitive data are stored on the IT network. The greatest cost to the organization is in this information leaking out, so the highest priority is confidentiality. Availability is last 
because a traditional IT staff would rather have their system go down than have sensitive information compromised.

Table 2: Priorities of IT and ICS Networks

\begin{tabular}{|c|c|c|}
\hline Priority & IT & SCADA/ICS \\
\hline 1 & Confidentiality & Availability \\
\hline 2 & Integrity & Integrity \\
\hline 3 & Availability & Confidentiality \\
\hline
\end{tabular}

Availability is the highest priority for ICS. Downtime of an ICS network could potentially damage expensive equipment as seen in the Stuxnet attack[6], damage the surrounding environment as seen in the Maroochy attack [3], deprive the community of critical utilities as seen in the Ukraine attacks[38], damage the company's reputation, or cause a loss of metering data, damaging the company's profits. Confidentiality is last because an ICS operator would rather have an attacker in the system snooping than to have any downtime. These factors present the challenge to security professionals. Security professionals face an infrastructure that was built before security was a concern, equipment that is old enough to be vulnerable to common attacks, and a zero-downtime mindset that makes applying updates and security patches difficult.

\subsection{Vulnerabilities}

The National Institute of Standards and Technology (NIST) separates ICS vulnerabilities into 6 categories: Policy and Procedural, Configuration and Maintenance, Architecture and Design, Physical, Software Development, and Communication and Network. Causes of security failures might overlap across categories. Specific systems may also have unique vulnerabilities as each ICS is specially designed. Some 
vulnerabilities can be removed or mitigated, while others must simply be accepted. See Special Publications 800-82[1] and 800-53A[39] for detailed analysis.

Policy and Procedural vulnerabilities are introduced into ICS through lack of security policies and a relaxed security posture in the organization. Security policies govern staff and stakeholders on proper use of systems to reduce the attack surface of the system. As shown above, security of ICS is often not the top priority so such policies can be scarce. Mitigations of this class of vulnerability include awareness and training programs to educate employees on proper upkeep of a secure environment, as well as maintaining a proper written security policy and plans for breaches. Proper authentication policies for employees such as smart cards and strictly enforced access policies, as well as proper authorization policies following the principle of least privilege as described in [37].

Misconfigured or default-configured devices make up Configuration and Maintenance vulnerabilities. NIST describes this class of vulnerabilities as those that would be similar to challenges faced by a corporate IT network; namely up-to-date patches of software and proper use of security controls available from vendors such as access control policies and firewall rules. The uptime requirements of some ICS networks as described in the previous sections can make patching and upgrading difficult, with some vendors recommending staying on outdated versions of software to ensure functionality or contractually obligating asset owners to involve the vendors in upgrades or risk voiding warrantees [32]. Legacy ICS components may be no longer supported, but still in production. Malicious software, or malware, is a common method of attack which can be mitigated. To go along with access control configurations, deficiencies in logging 
can prevent detection of abnormal behavior and make forensic analysis of attacks impossible.

Architecture and Design vulnerabilities arise from inadequate planning of ICS growth and failure to incorporate security priorities from the beginning of development. Legacy systems may have been designed before security technologies were widely available or may have expanded and changed without evaluating the effects of new capabilities on the organizations security posture. Loosely defined security perimeters around ICS networks make proper enforcement of security policies difficult. Intermixing of control and non-control network services and can cause control networks to be vulnerable to common non-control issues. A control network that depends on services such as Domain Name System (DNS) on an IT network might see reduced availability as an IT network typically does not conform to the same uptime standards.

Physical vulnerabilities range from physical access to control equipment to natural disasters. Improper access to network or control equipment could lead to theft, damage of hardware, unauthorized changes or additions to software and configurations of devices on the network, or installation of new unauthorized devices. Most devices, while properly access controlled from networked ports, have local ports with no access control capabilities to aid in maintenance. Consideration must be taken when securing safetycritical equipment to not make access to emergency shutdown functions too difficult for authorized personnel. Certain natural phenomena such as Electromagnetic Pulses, Radio Frequency (RF) interference, and power dips and spikes can cause temporary loss of service or permanent damage to devices and networks. 
Software Development vulnerabilities cover errors in the design and implementation of the software running in the environment. Fragile or bug-ridden software that has not been developed to high-assurance standards (or was developed before such standards existed) leave holes open for malicious or erroneous behavior to impact operation [40][41]. Specially designed ICS networks and components make patch release cycles difficult for vendors of ICS devices. Specific requirements for systems mean unique software patches made available for certain customers, each with their own testing cycles, leaving vulnerable components with no mitigations for extended periods of time. Software lacking security tools such as separate privileges and access controls also fit into this category.

Communication and network vulnerabilities that are present in traditional IT networks are present in ICS networks. Unsecured communication across the network or lack of a managed solution for restricting communication (such as proper firewalls) can open an ICS network to attack. There are cases specific to ICS networks though; such as use of proprietary protocols or encryption and simple embedded device drivers that are unable to handle anything but the most expected network traffic [41]. Previous sections described ICS networking protocol such as Modbus, but notably absent from the discussion of the base protocol was any form of authorization or authentication. These protocols are vulnerable to Man in the Middle attacks wherein a malicious actor intercepts communications, and to spoofing attacks wherein an attacker masquerades as a legitimate network device sending fake traffic. There have been efforts to retroactively add security to open protocols [7] and to update standards to include secure operating 
modes [23], but these still succumb to errors in design leading to more vulnerabilities [42].

Each of these classes of vulnerabilities have seen significant effort toward mitigation from both the private and public sector. Some of the mitigations include new technology and software developed to fill a hole in security capabilities, while others involve new methods for applying existing technology. There are numerous bestpractices guides [32], [1], [15], [39], [43] and whitepapers addressing each class that will be described in the next section.

\subsection{ICS Policies \& Best Practices}

The vulnerabilities described in the previous section have mitigating controls via both additional technologies and more strict policies. This section describes some of the industry standard best practices for software configurations, infrastructure designs, and human policy to harden Industrial Control System (ICS) networks against attack. The goal of policies is to reduce the effectiveness of attacks against existing vulnerabilities. As such they can be considered mitigating controls for cases where a security fix cannot be applied or does not exist. These also follow a defense-in-depth philosophy, working in tandem to boost the effective mitigation of the system as a whole. These recommendations come from National Institute of Standards and Technology (NIST) [1], [39], and industry group whitepapers.

\subsubsection{Systems Design}

The engineering of a system is more complex than ensuring each technical component is operating as intended. The definition of a system can change depending on 
context. Ross Anderson describes a variety of definitions in [44] and summarized below and applied to ICS.

1. A component such as a network card or cryptographic hardware.

2. A collection of the above plus an operating system, physical networking devices, and networking protocols.

3. The above plus applications that run on the nodes of the network such as an HMI.

4. The above plus operators.

5. The above plus management and corporate users

6. The above plus venders and customers

A system is more than its individual components. How the components interact with one another and how a system might allow the human element to compromise its integrity must be carefully considered.

The Physical Topology is the physical location and design of facilities in and around components of an ICS. In an ICS just as in traditional IT, if an attacker has physical access to a device then that device should be considered compromised. Physical security is just as important as electronic security and should be closely monitored. All doors should have locks, locks should be controlled with card readers, and physical security logs should be monitored just as closely as firewall logs. In highly critical systems armed guards may be necessary. Similarly, any computer devices used in the day to day operation of the ICS such as engineering laptops or PLC programming tools should never leave the area. Just as no unauthorized personnel should be allowed in, no operations equipment should be allowed out.

Physical topology can extend to environmental considerations for protections against mistake or malice. In the event a process is disrupted, having physical safeguards such as spillways to direct overflowing liquid materials and natural berms to prevent 
contamination outside of the area of operation. Designing physical systems to fail safe is an integral part of safety considerations, and can act as a means to mitigate certain security vulnerabilities.

Logical Topology, or Network Topology, is the design for the system's behavior and how a system's components interact with one another. This includes considerations for how networks are divided, restrictions on network access to certain areas and certain devices, and policies governing behavior of humans interacting with the ICS. ICS must be logically separated from any other network it is connected to. A demilitarized zone is recommended as a buffer between the corporate network and the ICS. This prevents traffic from flowing directly between the two networks. To further the separation separate sets of authentication credentials should be used for both networks. If a control engineer's credentials on the corporate network are compromised then the impact to the ICS is limited, if there is any impact at all.

The ICS itself must be split into multiple layers. An attacker should have to penetrate multiple levels of security before reaching the critical systems. This can be accomplished with firewalls on the drop of the ICS, between the control server and PLCs/RTUs, and between the historian and the remote substations. Individual components of an ICS must also be separated from one another. Traffic between the control server and a pump at one end of the plant should have no business touching the assembly line at the other end of the plant. Similarly the pump operator should not have access to send commands to the assembly line.

Traffic on an ICS can be more strictly defined than traffic on a traditional IT corporate network. With this in mind any extra functionality provided by devices on the 
ICS that is not being used such as extra radios, open ports, and web interfaces should be disabled. Not only does this reduce the attack surface of the ICS, but it also reduces the amount of monitoring and logging that needs to be done. The simpler nature of ICS also means operator roles can be more rigorously defined. Roles for operators should be designed according to the principle of least privilege [45][44]. If a lower level operator's credentials are compromised the breach will only affect the systems that operator is authorized to use.

\subsubsection{Configurations}

Components of an ICS network must be configured to suit their roles sufficiently within the design of the system. While many devices might not support security-specific features such as cryptography or access controls, they can be configured intelligently to reduce their attack surface and improve the security of the system as a whole. Additionally, for the devices that do support security specific features, special efforts should be made to ensure these features are properly enabled, configured, and tested. This section describes some of these configuration options and considerations.

Address Space Layout Randomization (ASLR) seeks to render shell code and return-oriented programming exploits difficult by reordering the memory addresses of elements of a program each time the program is run. ASLR is a setting that affects software in development. The software must have a specific linker flag at compile time to enable ASLR. ASLR is supported on Windows operating systems from Vista onward, FreeBSD, OpenBSD, Linux, Solaris, and OS X 10.7 onward. ASLR compliments data execution prevention (DEP) technologies. Where ASLR randomizes memory locations, DEP prevents execution of code from certain parts of memory that are commonly 
targeted by attackers, such as the heap and the stack. Support for ASLR and DEP is not common in embedded devices, with only $22 \%$ of devices supporting ASLR and $44 \%$ supporting DEP [46].

Application whitelisting is a method to eliminate the problem of having to track the changing malware trends by only allowing applications to run which have been given specific permission. Whitelisting software allows an administrator to specify which executables she wants enabled on the system. Malware that infects the system would never get a chance to execute. A case study from the Amor Group in 2012 reviewed the results of applying application whitelisting in the North Sea oil and gas industry [47]. Implementing whitelisting on the oil rigs, ships, and other assets revealed the presence of previously unidentified malware and helped the team catalog all of the legitimate software running on the multitude of computer systems. After whitelisting, no reinfections of systems were detected and a stricter management of applications was enabled.

As control systems gradually become less isolated, the edge of the network (or subnetworks if the control system is divided) must enforce proper access controls through firewalling. If the ICS cannot be air-gapped from the corporate network then strict control over all physical connections is essential to protecting the network. Firewall strategy for an ICS is similar to firewall strategy for a traditional IT network. When deciding on a firewall solution at minimum the firewall should require authentication before any configuration changes are made, be able to perform self-testing, and be able to perform logging. Firewall rules must be made according to the whitelist philosophy: traffic is denied unless it is explicitly allowed. This is vitally important because even the most 
innocent of traffic can cause problems to an ICS. A simple network enumeration, a perfectly harmless operation on a corporate network, can cause system outages. Considering how time-sensitive an ICS can be this "harmless" traffic can consume enough processing time to effectively render the nodes in the network unavailable. A firewalling strategy can be split between two separate methods, ingress and egress.

Ingress filtering means filtering network traffic coming into the network from the outside. Filtering incoming traffic is the first line of defense against malware infiltrating the ICS. There is very little traffic that should be entering the ICS. Traffic allowed to enter an industrial control system (ICS), if there is to be any allowed, can be clearly defined. Consideration can be given to the purpose of incoming traffic, from where the traffic originates, the communication protocols necessary, whether these operations can be done locally, and the time of day or week this traffic can be expected. These questions allow strict rules to be implemented and policies for temporary rules to be enforced.

Egress filtering involves filtering the network traffic leaving the network and originating from the inside. Since no system is unbreakable, it is important to implement firewall rules under the assumption that the system has already been compromised. A compromised system often makes outbound connections to a control server, either to push data or to receive further instructions. To this end it is necessary to filter outbound traffic just as much as inbound traffic. If malware finds its way onto the system through USB, such as described earlier with Stuxnet, then its damages can be limited by blocking its attempts to make connections outside the network. The same consideration should be made for egress filtering as ingress, with traffic leaving the network clearly defined before allowing traffic to egress. Another important consideration is whether the traffic 
needs to be part of a session, with packets traveling both in and out of the network to complete transactions. Special hardware, such as the unidirectional gateway described later, can be used to physically limit data to a single direction should this not be required.

\subsubsection{Patch Management and Disaster Recovery}

Because of the extremely high importance of uptime, change and patch management of ICS can be more daunting than traditional IT. Protecting the individual components of the ICS, the field devices, the historians, and the operation centers at the operating system and firmware level adds to the security of the ICS as a whole. If an attacker can compromise a single device by exploiting outdated or misconfigured firmware then that attacker is now in control of a trusted node and is now operating at that node's trust level across the network. However, an improperly tested patch can bring just as much harm as an outdated patch that has been compromised.

Proper testing of patches is necessary before the patches reach the production system. One of the major challenges in keeping an ICS up to date is the sheer number of variables that can be unique to a specific ICS environment. A vendor may not be able to tailor patches specifically enough to support a given deployment. It is possible a patch may do more harm than good if not tested well enough. A security patch released by a vendor must be thoroughly tested on a system as close to production as possible in functionality before deployment. This problem is not solely the responsibility of the control engineers. Vendors should be held to a higher standard in creating software and patches robust enough to handle the vast variability from system to system. 
Disaster recovery is the ability for a system to return to normal operation after an incident, whether accidental or by malice. Adverse conditions and disasters will happen and having procedures in place for these events is crucial to protecting the uptime of the ICS. This policy solution takes place in the planning stages of the ICS. The ICS must be designed in such a way that an unforeseen event can safely take down a part of the network with minimal effect on other parts of the system. To accomplish this each component of the system should be made redundant. If the first component goes down the second should be ready to instantly pick up the workload. An often missed component of redundancy is that components should fail in a way that does not result in diverted traffic overloading other systems either at the same stage of the process, upstream, or downstream. A graceful failure should be tested before the system goes into production. Should the entire system go down it is important to have a disaster recovery plan in place to get the system producing again. This can mean having a store of product in reserve while production is restored, or having multiple plants dispersed across multiple geographic regions which would be unlikely to be hit by the same natural disaster.

\subsubsection{Hardware Device Solution}

\subsubsection{Blue Coat ICS Protection Station Scanner}

Blue Coat's ICS Protection is a software and hardware solution that mitigates the risk of using USB storage devices on industrial networks. Stuxnet spread so successfully through removable drives and ICS Protection Station Scanner is designed to limit this specific attack surface. ICS Protection Station Scanner combines a hardware solution that resides outside the ICS and a software solution that runs on all Windows workstations 
within the ICS. Under Blue Coat's recommended policies any USB removable storage must be verified by its dedicated appliance before it can be used within the ICS[48]

\subsubsection{Unidirectional Security Gateway}

Waterfall Security's Unidirectional Security Gateway [49] addresses the problem of securely isolating the control network while also allowing business users on the corporate network to perform their job functions. This technology has been used to safely replicate the plant historian outside the network for the business to read. Transfer (TX) equipment sits inside the control network and queries the plant historian. The TX gateway then sends this data through a one-way fiber communication channel to an RX gateway sitting on the corporate network. The receive (RX) equipment then builds a faithful replica of the plant historian called a corporate historian on the corporate network. Corporate users and applications connect to the corporate historian to process the operating data. The one-way communication is enforced in the hardware of the gateways. The TX gateway only comes equipped with a laser, the RX gateway with only a photocell, and data is transferred through fiber. Sending data to the plant through this technology is not possible. A proposed network design for a unidirectional gateway is shown in Figure 3.

Another proposed use of this technology is allowing vendor support to troubleshoot problems on the control network without any actual remote access. A program records the local engineer's screen and sends that data through the unidirectional gateway to the vendor screen. The vendor directs any troubleshooting steps through telephone to the local engineer. In this scenario the vendor gets visual, real-time feedback from the system while also ensuring any actions are performed by a local plant engineer. 
- TX agent software queries servers \& gathers data

- Data \& metadata is sent to RX host

- RX agent software uses data to populate replica servers

- Faithful replica, tracks all changes, new tags, alerts in replica

- Corporate users and applications connect to replica

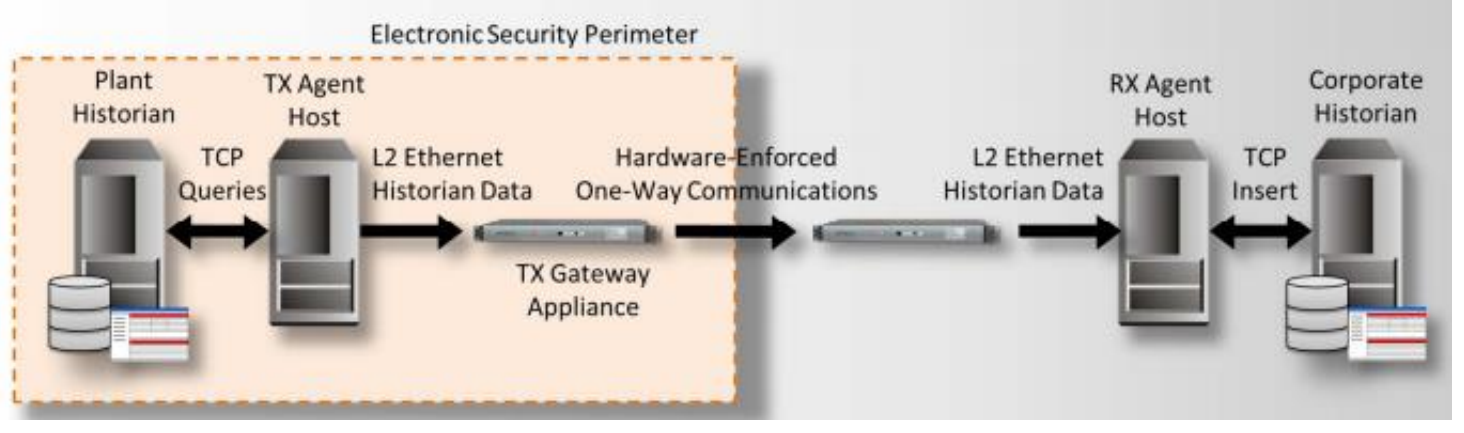

Figure 3: Proposed network design for a unidirectional gateway. Taken from [49]

\subsubsection{Tofino Xenon Security Appliance}

Tofino developed a security appliance specifically for SCADA environments that resembles a plug-and-play firewall. It is designed to operate between a process network and the business network. It is capable of filtering messages at layers 2, 3, and 4 of the OSI model, as well as performing deep-packet inspection to make filtering decisions based on the specifics of the control network protocol (Modbus, DNP3, Profibus, and the like) in use. The deep packet inspection of control network protocols is what separates the Tofino security appliance from a typical corporate firewall solution [50].

\subsection{Cyber Attacks}

The instances of the aforementioned vulnerabilities being exploited has been increasing recently as more Industrial Control Systems (ICS) have lost their isolation from their corporate network counterparts [40][30], [51]. This section describes some select attacks with information gathered from technical reports and forensic analyses of 
the attacks after the fact. This section describes three attacks: Maroochy water treatment facility attacks, the STUXNET attacks, and the Ukrainian power grid attacks. These incidents were chosen to highlight different threat actors, different vulnerabilities exploited, and different industries affected.

The Maroochy attacks in 2000 involved a formal employee of the asset owner using stolen equipment to remotely manipulate water treatment facilities. Vitek Boden, a disgruntled former employee of Hunter Watertech in Queensland, Australia, compromised sewage equipment to dump 800,000 litres of raw sewage into local parks and rivers [3]. Boden used intimate knowledge of the sewage system his former employer installed to enact revenge on both Hunter Watertech and Maroochy Shire Council. Boden drove from site to site over a 2 month period using stolen radio equipment to interfere with signals being sent between the control server and the RTUs in the remote substations. Boden would craft communication packets to spoof a station on the SCADA network and send out commands as though he were that station. Because there was no authentication processes present in the system Boden was able use this method to shut off pumps, disable communications between components in the system, and disable the alarms that would have alerted the plant operators to any suspicious activity. After an investigation, Hunter Watertech determined the problems were caused by a malicious attacker rather than faulty equipment. Boden was put under surveillance and eventually caught when stolen radio equipment was found in his car during a routine traffic stop [3]. This attack exploited poor or non-existent security measures from the Configuration and Maintenance class of vulnerabilities described above. Its complexity was relatively low; a former employee was able to exploit stolen equipment without needing to develop new 
software exploits or circumventing many security controls. As demonstrated with STUXNET, attacks on ICS networks can get significantly more complex.

Stuxnet was an elaborate malware targeting a specific configuration of ICS in 2009 and 2010. It is a definitive example of network isolation not guaranteeing safety [52]. Stuxnet gained infamy through its unprecedented level of complexity and because of its notable target in nuclear facilities. Roughly $60 \%$ of all infected hosts were found in Iran, with the remaining hosts spread across Europe, Asia, and the US. The worm was designed to reprogram a specific set of Siemens PLCs in such a way that the system being controlled would operate outside of its limits and degrade. It would also forge the operating data seen on the plant monitors so plant operators would not be able to detect any differences in the system. The complexity of the malware is readily apparent in the sheer breadth of its functionality. Symantecs Stuxnet Dossier lists the functionality described in Table 3, along with the category or categories of vulnerabilities the capability targeted.

Table 3: Stuxnet Capabilities and Targeted Vulnerability

\section{Stuxnet Capability}

Self-replicates through removable drives exploiting a vulnerability allowing autoexecution. Microsoft Windows Shortcut LNK/PIF Files (Automatic File Execution Vulnerability (BID 41732))

Spreads in a LAN through a vulnerability in the Windows Print Spooler. Microsoft Windows Print Spooler Service Remote Code Execution Vulnerability (BID 43073)

Spreads through SMB by exploiting the Microsoft Windows Server Service RPC Handling Remote Code Execution Vulnerability (BID31874). Copies and executes itself on remote

\section{NIST Vulnerability Category}

Configuration and Maintenance

Configuration and Maintenance

Communication and Network

Configuration

Configuration and Maintenance 
computers through network shares.

Copies and executes itself on remote computers running a WinCC database server.

\section{Copies itself into Step 7 projects in such a} way that it automatically executes when the Step 7 project is loaded.

Updates itself through a peer-to-peer mechanism within a LAN.

Attempts to bypass security products.

Exploits a total of four unpatched Microsoft vulnerabilities, two of which are previously mentioned vulnerabilities for selfreplication and the other two are escalation of privilege vulnerabilities that had yet to be disclosed.

Contacts a command and control server that allows the hacker to download and execute code, including updated versions

Contains a Windows rootkit that hid its binaries.

Fingerprints a specific industrial control system and modifies code on the Siemens PLCs to potentially sabotage the system Hides modified code on PLCs, essentially a Software Development rootkit for PLCs.
Policy and Procedure

Architecture and Design

Policy and Procedure

Communication and Network

Configuration

Architecture and Design

Software Development

Configuration and Maintenance

Policy and Procedure

Architecture and Design

Configuration and Maintenance

Policy and Procedure

Software Development
Configuration and Maintenance

Policy and Procedure

Software Development

Configuration and Maintenance

Policy and Procedure

This robustness suggest an immense amount of resources and person-hours poured into the product and perhaps hints at just how high value the target in Iran was to the authors. Symantec estimates a team of 5-10 developers and a team of management and QA engineers were required to produce the malware [52]. Not only did it require a lot of developers it also required a lot of ground work. Stuxnet used two compromised digital certificates and four 0-day vulnerabilities. It required a significant amount of reconnaissance on systems that were never connected to the internet. This means physical 
access to the systems was required to gather information on the infrastructure, then again to deploy the worm [6], [52]-[54]. STUXNET targeted state nuclear facilities and was not meant to spread outside of its specific target. Its collateral damage was limited and damage to civilian systems was limited. This care is not always taken as shown with power grid attacks in Ukraine.

A more recent attack on utility infrastructure occurred on the 23rd of December, 2015 [38], [55], [56]. This attack, launched in Ukraine, was the first publicly known cyber-attack targeting power infrastructure. The attack was able to disrupt power to 225,000 customers spread over 3 different service territories. The attack started off with a spear fishing campaign and an infected Microsoft Excel document loaded with BlackEnergy 3 [57], a malware toolkit that allows for connection to a command and control server. With BlackEnergy 3 the attackers were able to gain persistence on the power companies' business network and find their way through the VPN connecting the business and ICS networks. This attack involved at least 6 months of network reconnaissance and many steps of non-ICS related activities to reach the intended target. Once on the control network the attackers used built-in commands of the entity's RTUs to open the breakers in at least 27 substations to cause the outage. The attackers also overwrote the firmware of serial-to-Ethernet devices on the network to translate traffic from the operator's Human Machine Interface (HMI) to the Remote Terminal Units (RTUs) to block plant operators from issuing commands to restore the substations remotely. A telephone denial-of-service attack was subsequently launched on the companies' call centers to restrict the flow of information to the customers affected. On a global scale this attack was relatively small; only affecting 225,000 citizens for roughly 3 
hours. On a local scale this attack was catastrophic to the power networks and operators [38].

\subsection{Summary}

This chapter provided some insight into the motivations and challenges faced by industrial control systems and their operators. While safety has been a primary concern for control systems throughout their history, security has only recently become a priority. As control systems operators gradually opted out of air-gapping their networks in favor of better remote access, security researchers and malicious attackers alike have descended upon the field to find and document security holes in control network components new and old. A robust operator policy, intelligent network design, and utilization of security mechanisms can help mitigate potential threats, but a critical cyber-physical system with real-world consequences for failure requires a more formal approach. Chapter 2 introduces and discusses formal methods in software design and development to guarantee a piece of software will behave in a safe and secure manner, along with relevant research in the development of formal methods and its application to security and control systems. Chapter 3 presents a specific issue within control systems security and outlines the central contribution of this dissertation: a method of applying formal methods to control system security using TLA+ and seL4. Chapter 4 details the formal specification of a bump-in-the-wire security preprocessor, presented as a novel contribution to the field of control system security and as a proof on concept for the method. Chapter 5 discusses the mechanical model checking results of the specification. Chapter 6 lays out the design for the security preprocessor in CAmkES. Finally, chapter 7 summarizes these contributions and discusses possible avenues for future work. 


\section{CHAPTER II}

\section{LITERATURE SURVEY}

\subsection{Introduction}

Developments in formal analysis and security have occurred in tandem since the 1970s. Interest in the physical and logical security of data grew as mainframes and terminals gained widespread use in both the private sector and intelligence communities. Initially, government security efforts focused on finding and fixing software vulnerabilities through use of "Tiger Teams" [58], [59]. These teams consisted of computer experts acting as attackers, finding and exploiting software vulnerabilities then reporting their results so the vulnerabilities could be fixed. After many rounds of successful attacks, the Tiger Teams and security community at large concluded the cycle of finding and fixing security holes was futile - a secure system must be built with an intention to be secure from the start. Early steps of security research included finding a definition of secure, or more accurately a method of defining security for a given system. As can be seen in the research and development efforts in this chapter, formal methods help do each of the following things precisely:

- Describe a system's boundaries

- Describe a system's desired behavior

- Describe a system's desired properties

- Prove a system meets its specification

- Determine the circumstances under which the system does not meet its specification 
Funding from the National Security Agency (NSA) poured into the security community [59], and from there flowed into the formal methods community. The early research funded by the U.S. intelligence agencies culminated in the Trusted Computer System Evaluation Criteria, also known as "The Orange Book", first published by the Department of Defense in 1983 [60]. This document provided a metric for comparing the security posture of different computer systems, a guideline for vendors in the development of secure computer systems, and a means for specifying security requirements in government contracts. For example, for a system to be "A.1" certified according to "The Orange Book", the security requirements must be formally specified, the system must be formally modeled, and a formal proof must exist that the model meets its specification. The works described in this chapter are efforts to apply the A.1 certification criteria to an increasingly complex set of systems in an increasingly diverse set of scenarios, as well as efforts to bring a more formal approach to security of cyber physical systems.

\subsection{Formal Methods}

Progress of a society can generally be observed by the increase in complexity of its mechanisms, both social and technological. As complexity increases, it becomes easier to make errors in the design and implementation of systems -the accelerating rate of vulnerability reporting seen in [61] can attest to this. Formal methods are means for allowing engineers to develop increasingly complex systems while retaining a high degree of reliability. When a system is formally described, it can be better understood. Leslie Lamport, a pioneer in reasoning on distributed systems and inventor of Latex[62] 
and Temporal Logic of Actions (TLA)[63], says on pages 1 and 2 in his book Specifying Systems [64]:

Writing is nature's way of letting you know how sloppy your thinking is... Mathematics is nature's way of letting you know how sloppy your writing is... Formal mathematics is nature's way of letting you know how sloppy your mathematics is.

There are a variety of formal method techniques, each seeking to remove logical errors from systems. The four key types of formal method techniques discussed in this chapter are Model checking [64], Deductive Theorem proving [65], Abstract interpretation (also known as static analysis) [66], and Type inference [67]. Model checking involves creating a finite state machine that acts as a model of a real-world system and relevant propositions; the checker (human or machine) attempts to show that propositions hold in every state. Deductive theorem proving starts with a set of axioms and deduces properties of the system the axioms describe. Abstract interpretation tries to create an abstraction of code to form a less precise, but tractable model that can be reasoned upon. Finally, type inference is a completely automated method for deducing variable types at compile time and is available in many strongly-typed languages today [67]. Each of these techniques has its own strengths and weaknesses as discussed in the following sections.

\subsubsection{Model Checking}

Model checking creates a specification, or description of the system using the system's requirements as a starting place. The specification includes defining desired properties and structuring each individual piece within the system [68]. Formal specification introduces rigor using specification languages with mathematically defined 
syntax and semantics. Even though it is only the first step in the formal methods process, the act of formally specifying a design - describing a design precisely - can be enough to discover and remove inconsistencies and flaws in a non-formal design. Case studies using formal specifications at Oxford University, IBM, and Lockheed alone have shown reduction in production costs and improvement in code quality (fewer errors, earlier detection of errors) [11], [13], [14]. A specification can be as abstract or as granular as necessary, ranging from describing a perfect oracle that returns perfectly encrypted ciphertext to describing individual memory operations.

Model checking is a method for automating verification of specifications [69], [70]. A model checker typically comes with a custom language parser to allow an analyst to formally describe a system and requirements. A model checker requires the analyst to describe the system as a finite-state specification $\Phi$ and the desired property or set of properties to prove $\phi$. Such a system can be described using temporal logic and drawn out in Kripke structures [71]. Kripke structures take the form of $M=(S, I, R$, L) where $S$ is a finite set of states, $I$ is an initial state in $\mathrm{S}, \mathrm{R}$ is a subset of $\mathrm{S} \times \mathrm{S}$ such that $\forall \mathrm{s} \in S, \exists \mathrm{s}^{\prime} \in S,\left(\mathrm{~s}, \mathrm{~s}^{\prime}\right) \in R$ where $s$ and $s^{\prime}$ are individual states, and $L$ is an interpretation function that maps to the alphabet of states [69]. An example of a Kripke structure of a microwave is seen in Figure 4, taken from a lecture by Edmond Clarke on [69].

The properties to be verified can be described using linear temporal logic [72][73]. Equations can take the form of propositions such as $A \Rightarrow B$ (A implies B); Boolean operations such as AND, OR and NOT; and temporal operators. Temporal operators can be described with statements such as $X a$ for "a is true in the next state," $F a$ for "a will eventually be true," $G a$ for "a is globally true", and $a U b$ for "a is true until b 
is true." $G a$ can also be expressed as "a will be true in every state" and is called an invariant. This is sometimes called a safety property, as it specifies a dangerous state the system should never reach. The other operators can be used to describe liveness properties, or properties that are used to verify a system will eventually reach some set of states.

\section{Microwave Oven Example}

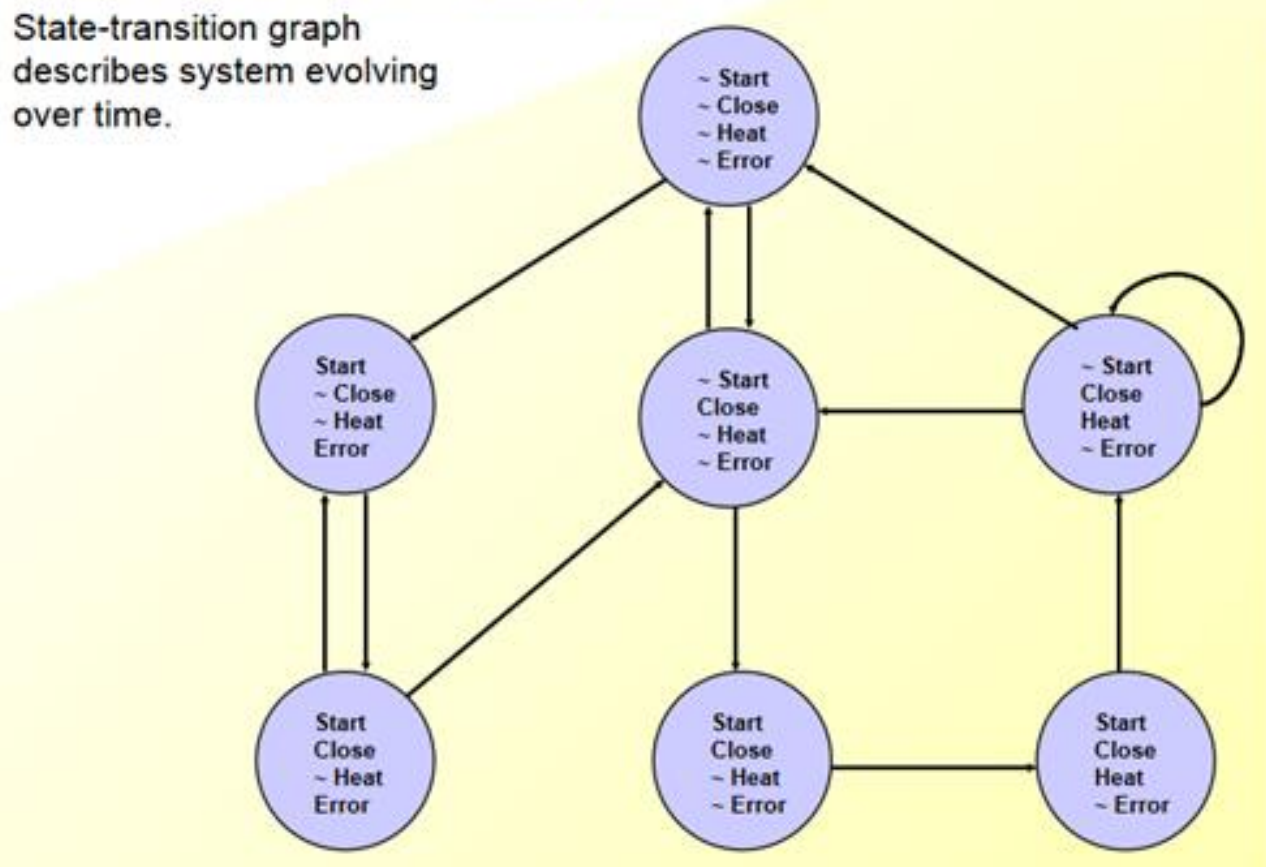

Figure 4: An example Kripke structure. Taken from [69]

The model checker can then automatically verify the property shown in Equation (1), that the specification models the requirements (i.e., the specification meets the requirements and desired properties). Model checking as a discipline is most useful when it finds counterexamples to requirements. If a model to be checked is not valid, a checker can discover difficult to find, easily checked, counter examples that can be fixed. However, if a model is valid, there is no easily checked method for the model checker to verify that. The most a model checker can say is that it could not find any 
counterexamples. Once a counterexample is found, an exact trace to the counter-example, or bug in the system being specified and checked, is available. This is useful when the nature of concurrent systems often produces exceedingly subtle bugs [possible ref to one such case].

$$
\phi \vDash \varphi
$$

This method has classically been used in the design phase of software development. Properties of the design can be reasoned on before any investment has been made into writing code. This allows for fundamental problems to be captured and fixed early and cheaply. This is not to say checking a model is easy. Even simple programs can suffer from the State Explosion Problem, wherein the number of states to be checked grows exponentially. Figure 5 shows the state space of a first-in-first-out (FIFO) queue algorithm containing more than 3800 states and more than 9600 transitions, with a maximum length of 3 elements taken from [74]. Such a small FIFO queue might not be practical, but it gives an example of the state explosion of even relatively simple programs.

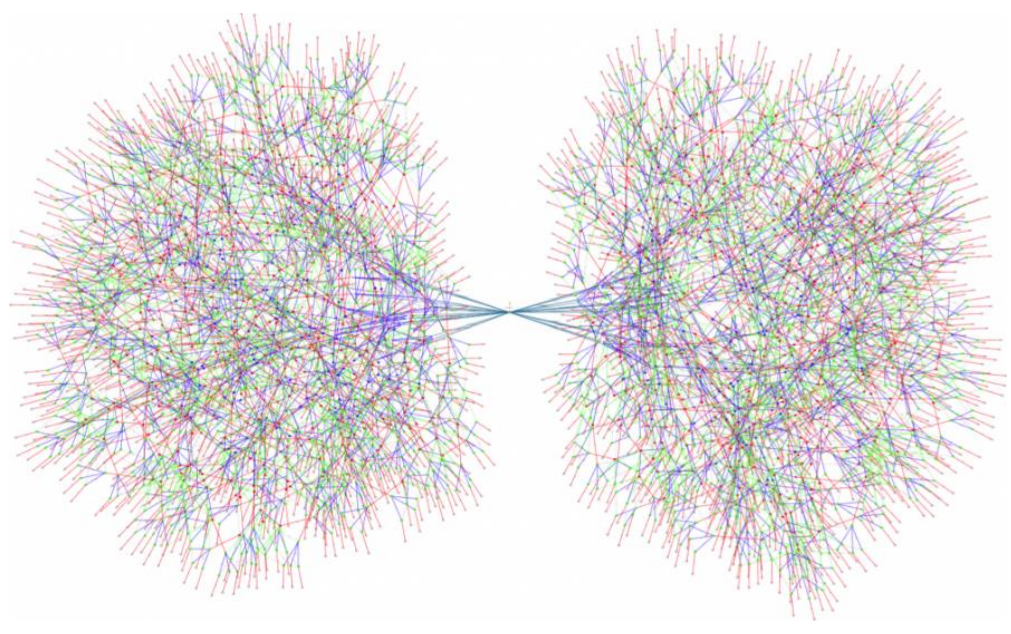

Figure 5:A FIFO queue capped at 3 elements 
The state explosion problem has seen significant research efforts. State explosion is present in parallelized systems, verification of which is paramount for adoption of such techniques in industry. An n-bit counter will have $2^{\mathrm{n}}$ states, while $m$ interleaved processes with $n$ states each will have $n^{m}$ states. Edmund Clarke opines that there have been four big breakthroughs in the state explosion problem thus far: Symbolic model checking with binary decision diagrams [75], partial order reduction [76]-[78], bounded model checking [79], and counter-example guided abstraction refinement (CEGAR) [80].

Symbolic model checking with ordered binary decision diagrams were introduced in the mid-1980s. Representing the states as a Kripke structure invited applications of graph theory into reducing the state space. Before this, states and transitions were represented explicitly with linked lists in memory as any directional graph might be. In 1986, Randal Bryant showed that larger specifications could be checked if their states and transitions were intelligently ordered into a binary decision diagram rather than a naïve linked list, reducing the states to be checked [75]. The binary decision diagram removes states and transitions that are implied in the specification and thus do not need to be explicitly written and computed, saving space and processing time. It should be noted that binary decision diagrams do not improve worst-case complexity of a model and in practice have been unpredictable in their complexity-savings.

Partial order reduction is a method for reducing the amount of redundant work done while processing models of concurrent systems. This method takes advantage of the commutative nature of some processes. Often, processing step A then step B will produce the same result as processing step B then step A. Using this property of some distributed processes, redundant branches of states can be eliminated from the necessary 
computation to exhaust the search space. Partial order reduction was developed independently in the early 90 s in [76]-[78].

Bounded model checking introduces satisfiability solvers to the model checking domain. Clarke et al. found that for certain properties, especially invariants, satisfiability solvers can find counter examples must faster than symbolic model checking and often without the exponential space requirement of binary decision diagrams [79]. The idea is to create a propositional formula from the states and transitions. This formula is Boolean - meaning its variables are either true of false and can be manipulated so the formula evaluates to either TRUE or FALSE. If the variables can be manipulated such that the formula evaluates to TRUE, the formula is said to be satisfiable. In bounded model checking, if the formula is satisfiable then a desired state can be reached within a set number of transitions. This method is somewhat restricting in that it can only test for certain properties. It is also not complete - at this time there is no way to know how long the bounded model checker must run before a counter-example is found if one exists.

Finally, Counter-Example Guided Abstraction Refinement (CEGAR), also known as localization reduction, is a means for automatically generating a model from a program, then automatically abstracting away unnecessary complexity within a model while checking [80]. The checking algorithm generates an over-approximation of the program that includes all the behaviors of the program by creating a state for every possible assignment of every possible variable. The transition relation is generated through the changing of variables in the program. If the specification holds on the abstraction, it is shown that it will hold for the concrete model. However, if a counter example is found, the algorithm checks if the counter example exists in the concrete 
program. If the counter example is found in the concrete program then this result is returned. If the counter example is not found in the concrete program, then it was introduced through the abstraction process. The abstraction is refined until the counter example is removed and the process repeats. A discussion of the advancements in CEGAR can be found in [80].

\subsubsection{Theorem Proving}

Theorem proving is the derivation of proofs from a formal system of axioms and inference rules. A developer expresses the program to be reasoned on and the desired properties of the systems as formulas then works to prove the properties from the given axioms within the formal system. Such work can involve deriving new definitions from the axioms and constructing intermediate lemmas to aid in the proving process. Though much of this work can be done by hand, this research focuses on automated and interactive (human-guided automation) theorem proving. Much of the work found in section 2.3 relies heavy theorem proving, and more discussion can be found in [65].

\subsubsection{Standards and Certifications}

Verifying systems can be prohibitively expensive. The larger the system the greater the expense of formal verification [11][81]. Full verification is not always necessary though and, depending on the system and use cases, value can be derived from a partial verification. Specific safety or security-critical properties of systems can be reasoned and proved at a reasonable cost. There are multiple industry- and field-specific standards for partial and full verification. 


\section{Common Criteria}

The NIST Computer Security Division started the Common Criteria Project to develop safety and security standards and certifications for software [82]. The goal of these standards was to improve the availability and efficiency of evaluating and verifying systems across IT systems. To facilitate this goal Common Criteria describes seven Evaluation of Assurance Levels (EAL). Each level builds on top of previous levels. Table 4 lists each level, a description of the requirements to achieve that level, and example of a product that has been certified at that level. More details of the process and requirements, as well as more examples of projects at each assurance level, can be found on the Common Criteria Portal [83], [84].

Table 4: EAL descriptions and example products

\begin{tabular}{|c|c|c|}
\hline Level & Description & Example \\
\hline EAL1 & $\begin{array}{l}\text { Functionally Tested - The system in question is } \\
\text { functionally tested for when security is not of great } \\
\text { concern. EAL1 provides evidence the implementation } \\
\text { functions according to the documentation. An } \\
\text { example of this would be a college senior capstone } \\
\text { project. }\end{array}$ & $\begin{array}{c}\text { Microsoft Windows } \\
\text { Vista }\end{array}$ \\
\hline EAL2 & $\begin{array}{l}\text { Structurally tested - a developer's cooperation is } \\
\text { required. This level introduces developer testing, } \\
\text { configuration management, and penetration testing. } \\
\text { This level describes typical software development } \\
\text { assurance requirements in industry today. }\end{array}$ & $\begin{array}{l}\text { Cisco's Remote } \\
\text { Access VPN }\end{array}$ \\
\hline EAL3 & $\begin{array}{l}\text { Methodically tested and checked - Procedures are } \\
\text { defined and followed throughout development to } \\
\text { meaningfully increase testing coverage. This level } \\
\text { also provides some assurance the system was not } \\
\text { tampered with during development. }\end{array}$ & $\begin{array}{l}\text { Huawei AR series } \\
\text { routers }\end{array}$ \\
\hline EAL4 & $\begin{array}{l}\text { Methodically designed, tested, and reviewed - } \\
\text { Maximum achievable level through good commercial } \\
\text { development practices, highest level likely attainable } \\
\text { by modifying existing software. This level has a high } \\
\text { security testing expectation, requiring demonstrating }\end{array}$ & $\begin{array}{c}\text { Oracle Enterprise } \\
\text { Linux version } 5 \\
\text { update } 1\end{array}$ \\
\hline
\end{tabular}




\begin{tabular}{|c|c|c|}
\hline & $\begin{array}{l}\text { resistance to a medium level attack. Assurance comes } \\
\text { in the form of a detailed design specification. }\end{array}$ & \\
\hline EAL5 & $\begin{array}{l}\text { Semi-formally designed and tested - This is the first } \\
\text { level that requires some specialized security } \\
\text { engineering and is beyond the scope of general good } \\
\text { development practices. Software that reaches EAL5 } \\
\text { likely was targeting EAL5 from the start of } \\
\text { development. Extra cost beyond the good } \\
\text { development practices is usually minimal. This level } \\
\text { requires semiformal design descriptions and a } \\
\text { structured, analyzable architecture. }\end{array}$ & $\begin{array}{l}\text { Samsung S3FT9PE } \\
\text { 16-bit RISC } \\
\text { Microcontroller for } \\
\text { Smart Card }\end{array}$ \\
\hline EAL6 & $\begin{array}{l}\text { Semi-formally verified design and tested - When a } \\
\text { higher cost for a high level of assurance and security } \\
\text { is acceptable. A formal model of the most important } \\
\text { security policies is required and a semiformal design } \\
\text { specification. The testing requirements grow stricter: } \\
\text { documentation of developer testing, independent } \\
\text { recreation of test results, and independent penetration } \\
\text { testing with a high level of attacker skill. EAL6 } \\
\text { represents a highly structured design, architecture, } \\
\text { and vulnerability analysis. }\end{array}$ & $\begin{array}{l}\text { Crypto Library } \\
\text { V3.1.x }\end{array}$ \\
\hline EAL7 & $\begin{array}{l}\text { Formally verified design and tested - The highest } \\
\text { level when security and safety assurance is of the } \\
\text { highest importance. EAL } 7 \text { requires full analysis using } \\
\text { formally verified design and implementation as well } \\
\text { as comprehensive and independently confirmed } \\
\text { testing procedures. }\end{array}$ & $\begin{array}{c}\text { Tenix Interactive } \\
\text { Link Data Diode } \\
\text { Device Version } 2.1\end{array}$ \\
\hline
\end{tabular}

$D O-178 B$

DO-178B is a standard for avionics software developed by Radio Technical Commission for Aeronautics. It defines five levels of safety criticality that help judge the priority that should be given to the assurance of software [85]. Table 5 lists and describes each level.

Table 5: Criticality Levels of DO-178B Standard

\begin{tabular}{|l|l|}
\hline Criticality Level & Description \\
\hline No effect & This software does not affect safety at all. \\
\hline Minor & $\begin{array}{l}\text { This software reduces safety or efficiency of airplane but not } \\
\text { beyond capabilities of the crew to handle without injury. }\end{array}$ \\
\hline
\end{tabular}




\begin{tabular}{|l|l|}
\hline Major & $\begin{array}{l}\text { Software with a Major Priority classification could cause } \\
\text { discomfort and possible injury to occupants. }\end{array}$ \\
\hline Hazardous & $\begin{array}{l}\text { Software in this category could cause potentially hazardous injury } \\
\text { to occupants. }\end{array}$ \\
\hline Catastrophic & $\begin{array}{l}\text { When judging avionics software, this category describes software } \\
\text { that, should it fail, would prevent flight and cause the deaths of } \\
\text { pilots, crew, and passengers. }\end{array}$ \\
\hline
\end{tabular}

\subsubsection{Limits of Formal Methods}

Formal program verification is an undecidable problem. Much like a system approaching infallible security, a system approaching complete verification will see its cost in time and resources also approach infinity for any non-trivial system. Formal methods are not an end-all answer to information security. A system can never be said to be completely secure and applying formal methods to verify a design does not change this fact. Formal methods allow only for a specific piece of a system to be mathematically described and its properties to be reasoned upon subjected to certain assumptions. As soon as these assumptions are violated, the equations and proofs cease to be useful. Because software is generally useful only when used in conjunction with other software, hardware, people, and environments, it becomes difficult to account for all possible external factors. A system is only as secure as its weakest link; a fully verified EAL7 application is only as secure as the kernel on which it runs, which in turn is only as secure as the hardware on which it runs, which is only as secure as the environment in which it runs, which is only as secure as the people who are running it. Formal methods are useful in increasing a system's security and robustness, but no system can ever be said to be $100 \%$ secure.

Formal methods have been time consuming and expensive in the past. Figure 6 and Figure 7 show 2006 U.S. Government Accountability Office estimates on the cost in 
money and time for climbing the ladder of common criteria levels. The figures show the resources required for just the evaluation piece of the certification for government projects that pursue the certification. Higher levels of verification may also negatively impact performance of an application as concessions might be necessary to model the system more efficiently and prove properties [81].

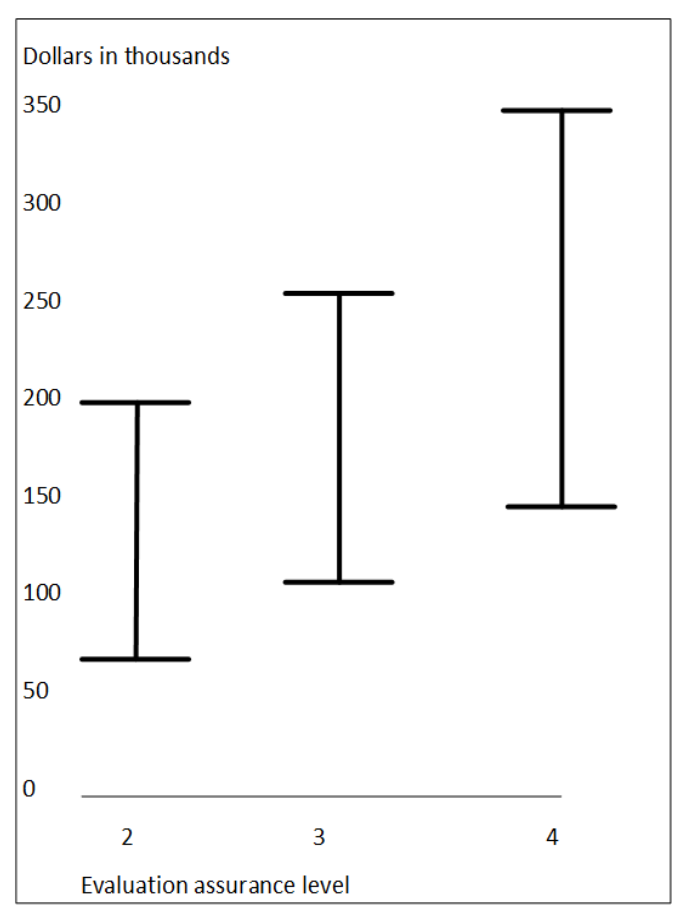

Figure 6: Range of costs required for completing product evaluations at various evaluation assurance levels. Adapted from GAO report [24]

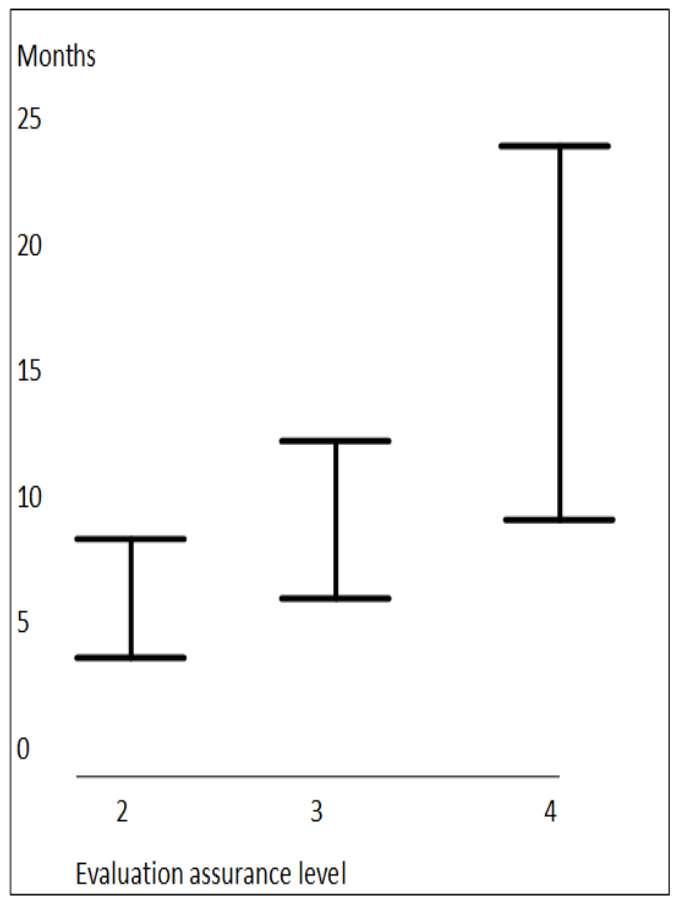

Figure 7: Range of sample cost of NIAP evaluations to vendors by evaluation assurance level. Adapted from GAO report [24]

\subsection{Verification}

Modern cryptography is an intersection between mathematics and computer science. Cryptography can be thought of in two parts: cryptographic primitives and cryptographic systems. A cryptographic primitive is the smallest piece of a cryptographic routine that has security properties (for example, a one-way hash algorithm or an encryption algorithm [86]). Cryptographic systems use primitives as building blocks to 
achieve security goals like keeping secrets and authenticating users. Primitives are difficult to mathematically prove secure, and usually rely on heuristic security arguments such as maturity of the algorithm and lack of weaknesses found by the community [87]. Cryptographic systems, on the other hand, can be proven secure when the primitives are assumed secure [88]. While this survey does not go into advances in cryptography, the research summarized in this section describes relevant advances is ensuring the code that implements the cryptographic primitives is correct.

\subsubsection{Verification of a Cryptographic Primitive: SHA-256}

Code that implements cryptographic primitives correctly is valuable in raising the level of available security, as a single correct implementation of a widely used primitive can be used everywhere. Andrew Appel presented his work on formally verifying SHA256, the Secure Hash Algorithm with a 256-bit digest [88]. SHA-256 is part of the SHA2 family of hash functions published by the NSA in 2001. Appel specifically looked OpenSSL's implementation of SHA-256, noting that because his proof work applies to the code and not to the algorithm itself, the many years of open-source scrutiny that OpenSSL has endured is still a valuable argument to its resilience against attack. The work shows, through a machine checked proof, that OpenSSL's SHA-256 correctly implements the formal specification of SHA-256 provided by the U.S. government in the Federal Information Processing Standards (FIPS) 180-4 Secure Hash Standard [89].

Appel's research served as a stress test for the Verifiable $C$ program logic for the C language and an example of how the Verified Software Toolchain (VST) can be practically utilized. Verifiable $\mathrm{C}$ logic has been proven to be sound with respect to the semantics of CompCert C, a subset of C digestible by the CompCert compiler [90]. This 
means code properties that have been proven in Verifiable $\mathrm{C}$ hold for the source code. CompCert has also been proven correct [90], meaning that properties that have been proven for the source code will hold in the compiled code. Thus, it can be shown that the compiled code for the SHA-256 algorithm satisfies its specification in Verifiable C. The proof process required started by building a functional specification, or a formalization, of the FIPS 180-4 standard in a mechanized proof assistant called Coq [91]. As this is a process done by a human, one might ask, "how can we trust that this formalization correctly describes the standard?" Appel points out that this trust is unnecessary, as even if the translation is incorrect, the properties described by the standard can still be proven in the functional specification. This work serves as a building block for future work on verifying higher order cryptographic functions, and on verifying entire cryptographic libraries.

\subsubsection{Verified correctness and security of OpenSSL HMAC}

Building on the SHA-256 verification work of Appel, Beringer et al. used a similar method to extend the verification to include OpenSSL's Keyed-Hash Message Authentication Code (HMAC) algorithm [87]. This research uses the FIPS 198-1 standard for HMAC [92] and verifies that OpenSSL's HMAC code correctly implements FIPS 198-1 as with the previous work, but goes further than previous work to show that the standard correctly holds its intended cryptographic properties.

HMAC is an authentication algorithm often used in communication protocols. When using HMAC, the sender of a message $m$ uses a secret key $k$ that has been preshared with the intended receiver. The sender computes the authentication code $s=H M A C(m, k)$, then attaches $s$ to the message. When the message reaches the receiver, it 
computes the authentication code $s^{\prime}=H M A C(m, k)$, then verifies $s^{\prime}=s$. Ideally, an attacker does not know $k$ and could not compute $s$. If the receiver determines $s^{\prime}=s$, it could trust that the message originated from the sender (as opposed to an attacker), and has not been tampered with. FIPS has requirements on the strength of the hashing function that can be used, but the HMAC specification can be generalized (without specifying the cryptographic hash algorithm used) as in Equation ((2.

$s=H A S H(($ opad $\oplus k)(H A S H($ ipad $\oplus k) m)$

The verification steps built off both Appel's work and earlier cryptographic proof work by Bellare on HMAC security properties [86], [93]. The first steps were to formalize specifications within the Coq proof assistant. The specifications are FIPS 198-1 and FIPS 180-4 for HMAC and SHA-256 respectively, Bellare's function for the HMAC algorithm (with pre-existing proofs), the API for OpenSSL header files for HMAC and SHA-256, and finally assumptions made about the security properties of the underlying cryptographic hashing algorithm. Next, the formalized specifications for Bellare's HMAC and the FIPS HMAC were shown to be equivalent, demonstrating that the security proofs derived from the earlier work apply to the later standard. Further, the formalized assumptions (common for pseudorandom functions) allowed new cryptographic security proofs to be derived from the standards. Finally, the process of translating from formalized specification into verified compiled binary ${ }^{1}$ is followed as described earlier. Beringer's research advanced the pursuit of a widely available, verified-secure, cryptographic library.

${ }^{1}$ The source, proof, and executable files can be found on Princeton's VST Github Repository (https://github.com/PrincetonUniversity/VST) 


\subsubsection{HACL*: A Verified Modern Cryptographic Library}

A collaboration between Microsoft Research and Inria has been pushing the stateof-the-art in cryptographic verification. In 2017, this collaboration presented the High Assurance Cryptographic Library (HACL*), a suite of formally verified implementations of cryptographic primitives and cryptographic systems [94]. HACL* specifically targeted the minimalist $\mathrm{NaCl}$ cryptographic library and API for verification work, as other libraries like OpenSSL are complex and unsuited to verification. NaCL (pronounced "salt") was developed to improving the state of the art on cryptographic library security, speed, and usability by simplifying and optimizing a core set of widely-used cryptographic functions [95], [96]. Tweet $\mathrm{NaCl}$, a particularly minuscule implementation of $\mathrm{NaCl}$, fits into 100 tweets and implements ChaCha20 and Salsa20 stream ciphers, the SHA-2 family of cryptographic hash functions, Poly1305 and HMAC authentication, Curve25519 elliptic curve encryption, and the Ed25519 elliptic curve signature scheme [97]. Each of these were verified and combined into a library of about 7000 lines of code that supports the $\mathrm{NaCl}$ API, and TLS-specific APIs used by OpenSSL, NSS, and miTLS.

Research on HACL* stated three goals: memory safety, functional correctness, and secret independence. Memory safety means the software never reads or writes at invalid memory locations. This can be achieved through strict coding practices such as ensuring no operation reads or writes past the last cell of an array and properly deallocating memory to leave no dangling pointers. Certain modern languages, such as Python and Java, include garbage collection techniques that manage memory for the coder and produces memory-safe code. However, this convenience is paid for with a performance reduction that can be unacceptable for cryptographic applications. The 
second goal, functional correctness with respect to the published standards, has been explained earlier. Finally, HACL* also strives for secret independence to eliminate certain classes of side-channel attacks. Secret independence is a coding technique that ensures:

1. Secrets cannot be used to decide what code executes next

2. Secrets cannot be used to decide what memory to access

3. Secrets cannot be used as input to instructions with a variable time

These methods ensure that a secret cannot affect how much time a particular piece of code takes to execute. By disconnecting the execution time from the secret, an attacker would not be able to weaken the security by timing inputs and outputs.

Microsoft and Inria's techniques differ from those previously discussed. Work on HACL* relies heavily on type-checking, instead of the automated theorem proving of previously discussed works, though theorem proving is still used when required. A formalized specification is still required and is created from the algorithm standards, but using $\mathrm{F}^{*}$ language (and its subsets) rather than Coq. Then, an optimized implementation is written in Low* (a subset of $\mathrm{F}^{*}$ that efficiently compiles to $\mathrm{C}$ ) and the proof work is done to show the implementation and formal specification are equivalent. Finally, the $\mathrm{F}^{*}$ code is compiled to verified $\mathrm{C}$ code with the KreMLin compiler. At the moment, KreMLin, the $\mathrm{F}^{*}$ type checker, and the theorem proving tool $\mathrm{Z} 3$ are unverified and must be trusted [94].

\subsubsection{Breaking and fixing the Needham-Schroeder Public-Key Protocol using FDR}

Data in motion can be particularly tricky to secure correctly. Sending an encrypted message from Alice to Bob, when both share the secret key and understand the algorithm, might be trivially easy to analyze, but network protocols are rarely so simple. What if the message is intended for more than one person? What if the different parties 
don't have a pre-shared secret key? What if the parties understand multiple encryption algorithms and must select one? How can one party be sure of the other's identity? How can the sender ensure the message that is received has not been tampered with? Answering any permutation of these questions can grow a network protocol beyond what can be trivially understood, and often lead to mistakes that break the security of the protocol without breaking the cryptographic primitives the protocol employs. The next two works represent formal verification efforts in networking protocols.

One of the foundational papers in formal analysis of secure networking protocols is Gavin Lowe's 1996 work on the Needham-Schroeder Public-Key Protocol [98]. Needham-Schroeder was used to establish mutual identity between two agents, an initiator A and a responder B. Public key cryptography is employed, meaning each agent has an associated public key which can be found on a public key server, and an inverse of the public key that is kept secret. This protocol also employs a nonce, a unique number included in the message to keep track of messages that have already been sent. The nonce is used to prevent an old message from being reused by an attacker. The important steps of the protocol can be described formally in Equation (3). $\mathrm{N}_{\mathrm{a}}$ and $\mathrm{N}_{\mathrm{b}}$ are nonces generated by $A$ and $B$ respectively. $\{m\}_{\mathrm{PK}(\mathrm{B})}$ indicates a message encrypted with B's public key. This message can only be decrypted using B's private key, thus ensuring only B can read the message.

$$
\begin{aligned}
& \text { Message 1. } \mathrm{A} \rightarrow \mathrm{B}: \mathrm{A} \cdot \mathrm{B} \cdot\left\{\mathrm{N}_{\mathrm{a}} \cdot \mathrm{A}\right\}_{\mathrm{PK}(\mathrm{B})} \\
& \text { Message 2. } \mathrm{B} \rightarrow \mathrm{A}: \mathrm{B} \cdot \mathrm{A} \cdot\left\{\mathrm{N}_{\mathrm{a}} \cdot \mathrm{N}_{\mathrm{b}}\right\}_{\mathrm{PK}(\mathrm{A})} \\
& \text { Message 3. } \mathrm{A} \rightarrow \mathrm{B}: \text { A. } \mathrm{B}\left\{\mathrm{N}_{\mathrm{b}}\right\}_{\mathrm{PK}(\mathrm{B})}
\end{aligned}
$$


Lowe modeled the protocol and checked it using Failures Divergences Refinement Checker (FDR). The attacker is assumed to have full access to messages traversing the network, but no ability to break encryption. All normal activities, such as decrypting messages encrypted with the attacker's public key and sending new messages are permitted. The attacker can also replay old messages, with or without knowing the encrypted contents of the message. The FDR checker produced a breach of security when checking that the responder (B) will only start a session with the initiator A if A took part in the protocol run. The model checker found this to not be the case, producing the steps seen in Equation (4) to breach the protocol's security guarantees. These steps show an attacker $I$ can use two different runs of the protocol to fool B into thinking it is communicating with A while sending messages to I. Lowe goes further to propose a fix to this vulnerability with proofs that the proposed fix works. Needham-Schroeder is a large component of widely used Kerberos cryptographic system [99].

$\begin{array}{rrl}\text { Message } \alpha .1 . & \mathrm{A} \rightarrow \mathrm{I} & : \text { A. }\left\{\mathrm{N}_{\mathrm{a}} \cdot \mathrm{A}\right\}_{\mathrm{PK}(\mathrm{I})} \\ \text { Message } \beta .1 . & \mathrm{I}(\mathrm{A}) \rightarrow \mathrm{B} & : \text { A. B. }\left\{\mathrm{N}_{\mathrm{a}} \cdot \mathrm{A}\right\}_{\mathrm{PK}(\mathrm{B})} \\ \text { Message } \beta .2 . & \mathrm{B} \rightarrow \mathrm{I}(\mathrm{A}) & : \text { B. A. }\left\{\mathrm{N}_{\mathrm{a}} \cdot \mathrm{N}_{\mathrm{b}}\right\}_{\mathrm{PK}(\mathrm{A})} \\ \text { Message } \alpha .2 . & \mathrm{I} \rightarrow \mathrm{A} & : \text { I. A. }\left\{\mathrm{N}_{\mathrm{a}} \cdot \mathrm{N}_{\mathrm{b}}\right\}_{\mathrm{PK}(\mathrm{A})} \\ \text { Message } \alpha .3 . & \mathrm{A} \rightarrow \mathrm{I} & : \text { A. I. }\left\{\mathrm{N}_{\mathrm{b}}\right\}_{\mathrm{PK}(\mathrm{I})} \\ \text { Message } \beta .3 . & \mathrm{I}(\mathrm{A}) \rightarrow \mathrm{B} & : \text { A. B. }\left\{\mathrm{N}_{\mathrm{b}}\right\}_{\mathrm{PK}(\mathrm{B})}\end{array}$

\subsubsection{Implementing TLS with Verified Cryptographic Security}

The original communication protocol of the internet, Hypertext Transfer Protocol (HTTP), did not include any means for encrypting transmitted data, requiring sensitive information such as usernames and passwords and credit card information to be sent in plaintext. Secure Hypertext Transfer Protocol (HTTPS) is the current solution for securing general internet traffic. HTTPS is built using Transport Layer Security (TLS), TLS is itself is a suite of many cryptographic primitives and systems. The wide variety of 
implementations, supported protocols, and versions of TLS installed on servers leaves the state of web security an unmanageable mess. Project Everest is a collaboration between Microsoft Research and Inria to transform the formal specification for the newly finalized TLS 1.3 standard into a portable library [100]. This project produced many relevant tools and supportive libraries to aid in the proofs and translations required to produce verified assembly. The scope of the Everest project can be seen in Figure 8.

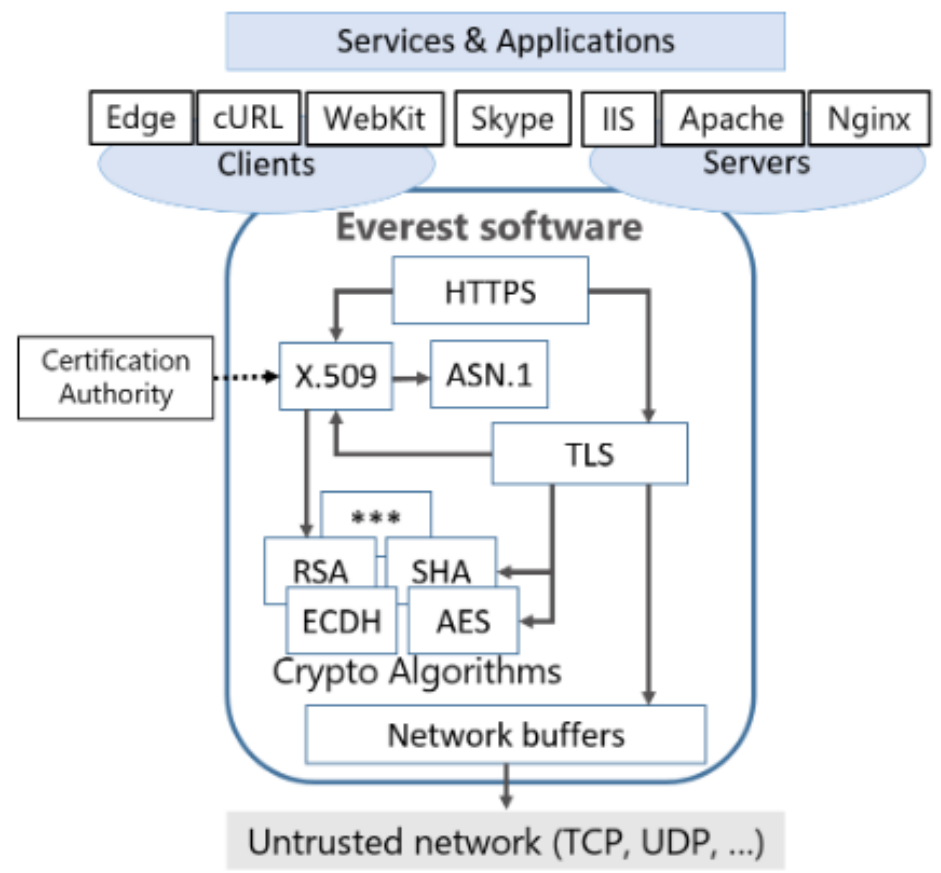

Figure 8: The verified components of the TLS1.3 suite. Image from https://projecteverest.github.io/

The verified libraries are only half of the contributions of the Everest project. The authors note that software is not static; as the years pass and the web landscape evolves, changes will need to be made to the TLS 1.3 standard, then added to the Everest portable libraries. Changing verified code is not easy, and few organizations are capable of modifying verified code without compromising the proofs and properties. The Everest team will probably have to continually support the project, but future projects might be 
undertaken by less-funded organizations with fewer verification experts thanks to the supported tools developed alongside. These tools include:

1. HACL*: Seen in section 2.3.3

2. Low*: a subset of $\mathrm{F}^{*}$ targeting low-level programming that allows finer optimizations

3. KreMLin: a compiler (unverified currently) that extracts Low* to C

4. Vale: A tool for writing verified and high performance assemble code

\subsubsection{The Temporal Logic of Actions, TLA+}

Early research in formally specifying software focused on sequential actions and produced tools that could express and reason on sequences with an acceptable level of complexity. These tools and approaches struggled when tasked with modelling concurrent systems [63]. Efforts to extend these tools to be applicable to concurrent systems, usually by replacing predicate calculus with temporal logic, could not reduce the computational complexity to be practical enough for use. Temporal reasoning on an entire system can be prohibitively expensive. In 1994, Leslie Lamport presented his work on modelling concurrent systems through use of actions [63]. While work focused on a single state of a specification, Lamport's actions comprised assertions about pairs of states. Temporal Logic of Actions allows much of the temporal reasoning that was consuming computer power and brain power to be replaced with standard, non-temporal, reasoning about actions.

TLA+ is a formal modeling language developed by Leslie Lamport with a focus on modeling and reasoning on concurrent systems [64][101]. PlusCal is an "algorithm language" used to model algorithms in a much more expressive fashion than a typical programming language. Both TLA+ and PlusCal use mathematical notation to expand the reach of a model beyond a programming language to allow for more rigorous definitions 
and descriptions of algorithms and systems[101]. The inclusion of mathematical notation also facilitates model checking and proofs on properties of systems and algorithms. PlusCal, a more programmer-friendly route into the TLA+ toolchain, can be automatically translated into TLA+ and used with the TLC model checker. The TLC model checker can be described as a brute-force checker that will explore all states up to a certain number of state transitions and alert on any properties that have been violated and give a trace of the steps to violate such property.

\subsubsection{Use of Formal Methods at Amazon Web Services}

Amazon has published at least two experience reports on using formal methods in the design of their web services platform, specifically with TLA+ [102], [103]. Amazon has found multiple benefits while incorporating formal specification into their development process. The first and likely most obvious is finding existing bugs in their platforms. Table 6 lists some of the results of checking the formalized models of systems within Amazon's ecosystem, with multiple bugs usually found in less than 1000 lines of specification code. The second benefit is an increase in understanding of designs for new systems, or new features for existing systems. Instead of building a naïve design then modifying it to handle what might go wrong, the new design process places more focus on "what needs to go right" from the start. This prevents edge cases from presenting new and unimagined ways for the systems to fail. A third benefit described in the experience report is that engineers could proceed with applying changes, whether increasing scalability or increasing existing performance, with greater confidence. Downtime in production services is expensive, and the risk of extended downtime must weigh into the analysis of whether an upgrade is worth the cost. Making the proposed changes to the 
formal specification and rechecking that all desired properties hold has given Amazon engineers a way to achieve greater confidence in upgrades to production systems. An optimization can only be applied if it does not introduce any problematic edge cases; formal specification and analysis give added proof that even the most aggressive of optimization are safe. Finally, the experience report describes the formal specifications as useful in documenting the systems. The specifications act as precise reference for engineers to communicate ideas and allow new engineers to learn about the system quickly. The documentation is essentially "free", as it is a byproduct of the formalization process.

Table 6: Benefits of TLA+ in Amazon's Ecosystem. Adapted from [103]

\begin{tabular}{|c|c|c|c|}
\hline System & Components & Line Count & Benefit \\
\hline \multirow[b]{2}{*}{ S3 } & $\begin{array}{l}\text { Fault-tolerant low-level } \\
\text { network algorithm }\end{array}$ & 804 PlusCal & Found 2 bugs \\
\hline & $\begin{array}{l}\text { Background } \\
\text { redistribution of data }\end{array}$ & 645 PlusCal & $\begin{array}{l}\text { Found } 1 \text { bug, and } \\
\text { found a bug in the first } \\
\text { proposed fix }\end{array}$ \\
\hline DynamoDB & $\begin{array}{l}\text { Replication \& group } \\
\text { membership system }\end{array}$ & 939 TLA+ & $\begin{array}{l}\text { Found } 3 \text { bugs, some } \\
\text { requiring traces of } 35 \\
\text { steps }\end{array}$ \\
\hline EBS & Volume management & 102 PlusCal & Found 3 bugs. \\
\hline $\begin{array}{l}\text { Internal distributed } \\
\text { lock manager }\end{array}$ & $\begin{array}{l}\text { Fault tolerant } \\
\text { replication and } \\
\text { reconfiguration } \\
\text { algorithm }\end{array}$ & 318 TLA+ & Found 1 bug \\
\hline
\end{tabular}

\subsection{Modeling and Verification of Operating Systems}

Achieving verified secure control systems is a game of compromises. Formal analysis is a difficult process and it can be infeasible to verify every piece of code that could run, or even every physical process that could occur. As such, current state-of-theart tries to strategically apply a formal approach to only the most critical pieces or makes strategic compromises to performance metrics to attain a simpler and more verifiable 
design. Some designers may only wish to verify certain properties of processes, while leaving others to traditional testing methods. An operating system presents a level of complexity that must be strategically planned for and verifying operating system properties have produced valuable insight into the problem of verifying security at a large scale. Presented here are examples of formal methods applied to operating systems.

\subsubsection{The Bell-La Padula model}

The state of computing in the 1960s and 1970s encouraged time-sharing strategies for companies and agencies that had trouble covering the costs of monolithic mainframes on their own. For agencies that handled classified data, a separate mainframe was required for each of the independent security levels. While time sharing presented a major cost-saving opportunity, it also presented a novel risk in handling classified processes, as multiple security levels would run on the same hardware and data. A high degree of assurance that processing artifacts of each security level was kept separate was required. There are many examples of insecure software and systems that were built only focusing on function, that patch security holes after-the-fact [61], [104]-[106].

David Bell and Len La Padula, as part of MITRE, were involved in early research on defining a "mathematical model of security in computer systems". The long-term goal was to formally specify what security meant in a computing environment, and to build computing environments from the ground up to meet such a definition. The Bell-La Padula Model [59] established three rudimentary properties that must hold for a mixedclassification system to be considered secure: simple-security (the user hold an equal or greater security clearance than the object he or she is trying to use), discretionary-

security (the user has been granted permission to use the object), and the *-property 
(sometimes known as the "No-Write-Down" property, where the user is not able to transfer information to a security object of a lower specification). Any rules governing the changes of the system's state (accessing objects, creating objects, granting access to another user, among others) must be proven to preserve these three properties. A system with state-transition rules proven to preserve the three properties thus stayed in a secure state, assuming it started in a secure state.

Formal models require refinement before they are worth anything more than the paper they are written on. Bell and La Padula's model gradually matured as attempts were made to put its rules into practice. Originally, the time required to check all the objects a user is currently accessing when a request is made to access a new object was punishing. Certain questions were left unanswered in the early versions of Bell-La Padula like what level of clearance should be assigned to a task scheduler that must both read and write multiple clearance levels simultaneously (thus violating simple-security and star-property) when swapping jobs. These engineering problems forced the Bell-La Padula model to more and more accurately describe a real-life computer system. More rules were added, rules and state-transitions became more nuanced, subjects became differentiated. Demands at the time pushed the model to more accurately describe the Multics operating system [45] in particular.

In his Look Back on the Bell-La Padula Model [59], David Bell notes the benefit of the back and forth communication between himself in his modeling role and the engineers implementing the design, both in simplifying the general model, and in tailoring the model to ease some of the engineering challenges that were encountered. He also acknowledges the pace of software development has harmed the security posture or 
systems, despite advances in security technology. Easing the burden of verified security, while also performing "selfless acts of security in the form of crafting and sharing reference implementations of widely needed components" as David suggests, would be common themes in the research that followed.

\subsubsection{The transfer of information and authority in a protection system}

Proposed in 1977 by Richard Lipton and Lawrence Snyder [107], take-grant consists of subjects, objects, a finite set of access rights, and a finite set of rules for distributing the access rights. The safety analysis then determines if, given the set of rules and initial distribution of access rights, whether a subject could ever be granted some specific right it did not originally possess. Take-grant is decidable - that is, the safety analysis can be completed in linear time. This model can be represented as a directed graph, with subjects and objects as nodes, and permissions (capabilities) as edges. The node from where the edge originates has authority (as defined by the label of the edge) over the node to which the edge terminates. Take-grant and its variations/refinements have many different rules, but four rules are fundamental (images adapted from [107]):

Take: If a subject $\mathrm{s}$ has take capability $t$ over an object $\mathrm{x}$, the subject can assume any capabilities the object possesses.
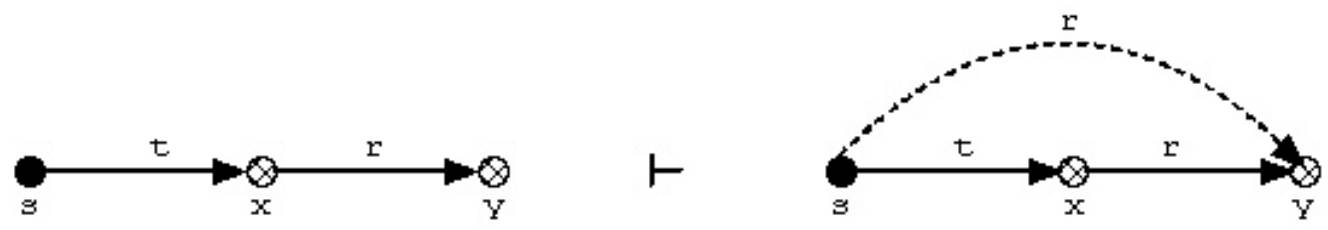
Grant: If a subject $\mathrm{s}$ has grant capability $g$ over an object $\mathrm{x}, \mathrm{s}$ can share any of the capabilities it posses with the $\mathrm{x}$.
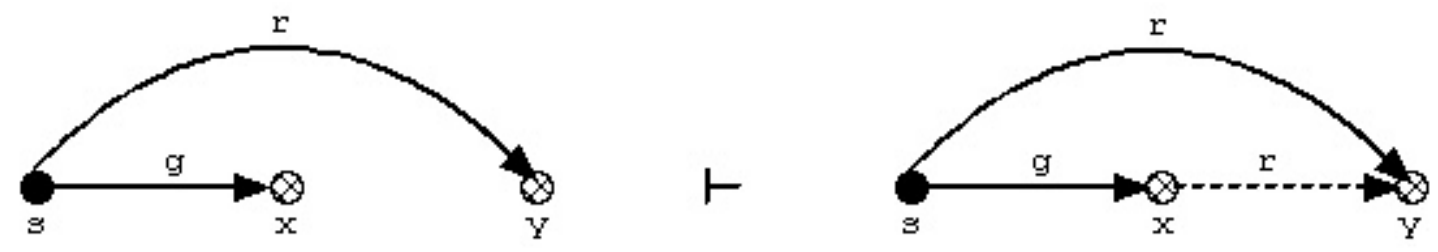

Create: A subject s can create a new node on the graph $\mathrm{x}$ with a subset of capabilities $\mathrm{p}$ from the set of possible capabilities.

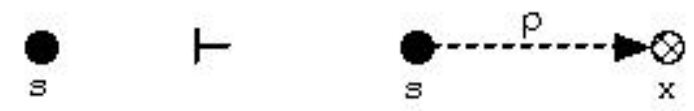

Remove: A subject $\mathrm{s}$ with a set of capabilities $p$ over an object $\mathrm{x}$ can delete a set of capabilities $r \in p$. The resulting capabilities $\mathrm{s}$ has over $\mathrm{x}$ can be described as $p-r$. If $p-r$ is empty, the edge is deleted.

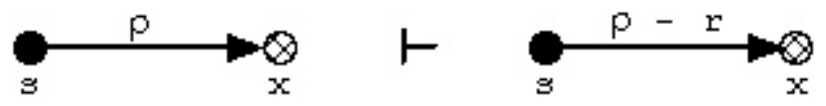

Lipton and Snyder's work was expanded by Bishop et al in a 1979 paper to include analysis of de facto capabilities and de jure capabilities [108]. These cover capabilities that can be obtained through some combination of take and grant capabilities of other subjects (de facto) and capabilities that can be indirectly exercised through other nodes on the graph (de jure). An example of de jure is a graph with 3 nodes $\mathrm{S}, \mathrm{X}$, and $\mathrm{Y}$ wherein $\mathrm{S}$ can write to $\mathrm{X}, \mathrm{X}$ can write to $\mathrm{Y}$, but $\mathrm{S}$ cannot write directly to $\mathrm{Y}$. The 
information $\mathrm{S}$ is trying to write can be passed through $\mathrm{X}$, then to $\mathrm{Y}$. In this case, $\mathrm{X}$ can be called a co-conspirator.

\subsection{3 seL4: formal verification of an OS kernel}

As with Bell-La Padula with Multics, Data61, under The Commonwealth Scientific and Industrial Research Organization (CSIRO, formerly NICTA), refined and evolved the take-grant security model to prove components running on the same hardware could be logically isolated from one another for its secure embedded L4 (seL4) microkernel series [10], [109], [110]. The seL4 security model modified the create rule of the original take-grant model. After boot, all memory that has not been pre-allocated for the kernel is divided into untyped memory (UM) objects. A resource manager outside of the kernel has a full rights over each of the UM objects, meaning that all memory is accounted for with capabilities. With this in mind, the create rule used in seL4's model requires the subject to have the create capability over some UM object. This operation is called retype, as it takes an existing object and transforms it into something useful to the subject. Retype is restricted to ensure no overlapping of retyped objects, and no previously retyped objects within the memory region being retyped. The seL 4 security model makes a few other less significant changes. Its remove rule does not modify an existing edge in the graph. As capability lists within seL4 are immutable, an edge must be deleted then recreated with the desired set of capabilities. Revoke is an operation to remove a set's capabilities at once, though this can be thought of as a sequence of remove operations. Finally, the seL4 security model does not include a take rule. A subject can give capabilities, but cannot take capabilities. 
Refinement of the seL4 security model to the seL4 microkernel involved proving that each operation that the microkernel can perform can be mapped to a single or sequence of rules in the security model. Thus, each state the microkernel can find itself in can be represented in the security model, and shown to hold the any properties guaranteed by the model. One such property is isolation. Data61 was able to show that a subsystem, a set of connected entities within the graph, is not able to gain a capability over an entity inside another subsystem if that capability was not already present. Additionally, they were able to show that if that capability was already present, it could not be increased. Practically, as the kernel operations have been shown to refine the model rules, this means components running on top of the kernel have proven isolation properties. Proofs were completed in the proof assistant software Isabelle/HOL [111].

Every line of code within seL4 is proven to behave exactly as the specification intended, with a focus on performance and security. Some compromises were made, such as making no guarantees with regards to timing of execution, aggressively pushing functionality out of the kernel and into user space where possible to reduce the codebase, and a slight performance drop from non-verified microkernels. Despite these compromises, the microkernel provides valuable guarantees with respect to safety, security, and reliability.

The seL4 microkernel has proofs of stability through use of invariants. Data61 recognizes four categories of invariants used in the proof work: low-level memory, typing, data structure, and algorithmic invariants. Memory invariants include no objects at memory address 0 and kernel objects do not overlap. The type invariants ensure every kernel object has a well-defined type, and that references point to objects of the correct 
type. The data type invariants ensure data cannot be corrupted by sloppy construction of structures like linked lists. Examples of data type invariants are no loops in pointer structures and that lists are always terminated with NULL. The final category is invariants more specific to the operation of seL4, such as removing the overhead of a runtime check by proving the condition being checked is always true. The seL4 team notes that cleverness was needed when working with operations that delete or retype objects but have shown that the kernel is not able to perform unsafe operations.

\subsubsection{The HACMS program: using formal methods to eliminate exploitable bugs}

The Defense Advanced Research Projects Agency (DARPA) has been looking at ways to practically apply formal methods research to current and legacy projects to bolster the resilience of U.S. military systems to cyber-attacks. In the past, researchers had to develop their own tools to tackle the specific case of software they were trying to verify. Often, the tools would be just as valuable to the community as the verification work. However, the steady increase in formal methods infrastructure, that is the rise in the level of expertise, the improvement in proof automation techniques, the exponential increase in computing power available, and the maturity of tools, has finally brought the techniques into the realm of practicality. In the experience report in [112], Fisher et. al. describe DARPA's High Assurance Cyber-Military Systems (HACMS) program and its efforts to apply verified components to existing, unverified systems [112].

HACMS research began with an open source quadcopter. A red team (professional hacking team) tested the security of the quadcopter and demonstrated multiple mission-critical vulnerabilities, eventually gaining full control and flying the quadcopter. Then, the HACMS team refactored the quadcopter and formalized its design. 
The new design had proof of several security properties: memory safety, resilience against malformed or unauthenticated messages, and that any authenticated and wellformed message from the control station will eventually reach the motor controller. Once implemented, the red team was given six weeks and full access to all the design documents of the systems with the goal of wirelessly disrupting the operation of the new quadcopter but were unsuccessful.

The next stage of research involved a Boeing Unmanned Little Bird helicopter and additional constraints of not being able to refactor the hardware of the system as they had with the quadcopter. The goal was to retrofit the Little Bird with verified components to increase its resilience to cyber-attacks with the acknowledgement that not every piece of software in legacy systems could be verified. For this system, HACMS employed the seL4 microkernel to act as a layer between the hardware, the verified software components responsible for communication with the control station, and the unverified components responsible for the mission cameras. The isolation guarantees provided by seL4 were leveraged to ensure that even though certain components within the helicopter might be vulnerable, they could not be used to compromise the components critical to the mission. After the retrofit, the red team was given root access to the mission camera component and tasked with disrupting operation of the helicopter in general. The red team was able to destroy the component they were given, but was not able to pivot from that component to control others or cause a cascading failure affecting other components [112]. 


\subsection{Cyber Security for Control Systems}

Control systems have a notoriously low-priority view of cyber security. Many popular standards for control network design and protocols were developed and implemented before cyber threats were prevalent enough to consider. The Maroochy attack described in [3] was the first known attack on a control system, and since then the landscape has seen more frequent [113], [114] and more sophisticated [57], [115] attacks. The nature of control systems requires security mechanisms to be reliable on the order of decades, with little tolerance for disruptions in day-to-day operation. Formally verified security systems, with precisely understood behavior, then become valuable tools in both retrofitting existing system and designing new systems to be resilient to cyber-attack. Works in this section apply a formal approach to control systems security.

\subsubsection{Formal Vulnerability Analysis of a Security System for Remote Fieldbus Access}

In 2011, Cheminod, Pironti, and Sisto presented their formal analysis of vulnerabilities in a secure remote fieldbus access system [116]. The system to be analyzed is depicted in Figure 9. Communication with the fieldbus is initiated by users in the corporate network and is policed by the gateway (GW) according to access control lists. A hierarchical symmetric key system is used to achieve confidentiality, integrity, and authentication: there is a single domain key from which each gateway derives the Gateway User Authentication key (GUA) and the Gateway User Encryption key (GUE). From these, each user derives a unique User Authentication key (UA) and a unique User Encryption key (UE). Each gateway shares the same GUA and GUE, and each gateway and user stores their own key-pairs locally. A user can use the same key-pair to interact with any gateway. 


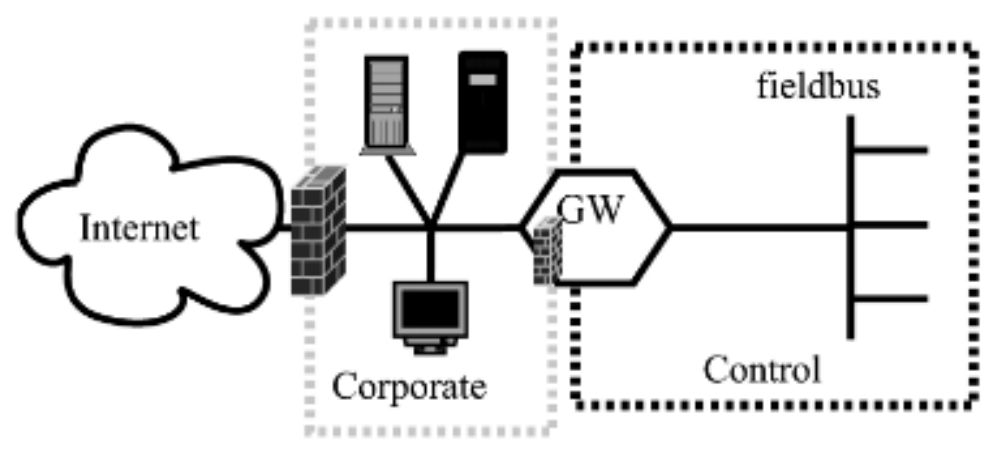

Figure 9: A sample fieldbus architecture, from [116]

The protocol was modeled using ProVerif [117], with a User role and a Gateway role performing the necessary actions. The authors noted that previous papers only informally described the protocol, so formalizing it required making design choices where the original specification was not clear. The security goals of the protocol also needed to be formalized within ProVerif and can be informally described as such:

Privacy:

Given an attacker that is able to see all traffic on the network and produce new messages, the attacker must never know the identity of a sender that is not itself, the data in the request, or the response to the request.

Authentication:

Whenever a gateway receives a message from the user, the user previously and intentionally sent that message.

An attacker is not able to forge a valid message.

Integrity:

A response is only valid if it is a response to the originating request.

Of these properties, the authors found that only the privacy could be proven to hold; the remaining were proven to not hold. The results produced by ProVerif showed that this protocol is susceptible to replay attacks at multiple steps during the transaction. An attacker could fool the gateway into thinking it is communicating with a valid user by 
replaying a previous request within 150 seconds. An attacker can also replay a request back to the user as though it were a response from the gateway as the request and response share the same format.

Next, the authors formalized the entire system infrastructure to analyze security of a particular network configuration using a Prolog-based tool and approach. At this level of abstraction, the protocol is assumed to be flawless at first, then flawed as described above, to determine if a given networking configuration exposes the vulnerabilities to attack. A state-transition system was used, defining an initial state of the network then allowing the status of the network to evolve as actions were performed. Analysis of this model involved determining if any state could be reached such that the security properties were violated. The analysis determined that a flawless protocol left the system in a safe state regardless of transitions, but the flawed protocol allowed invalid operations to occur.

\subsubsection{Towards Formal Security Analysis of Industrial Control Systems}

Marco Rocchetto and Nils Tippenhauer have extended the Dolev-Yao model for interactive cryptographic protocols to suit the needs of control systems - specifically, a water treatment plant [118]. The new model is then used to find potential attack vectors of the control systems through formal analysis. The Dolev-Yao models an attacker with full access to the network, but who is unable to break the cryptography [119]. Extensions by Rocchetto and Tippenhauer and related works add new attacker profiles that are more specific to control systems, as well as rules that govern the interactions between the software and hardware (like opening a valve), and rules governing physical capabilities

of the attacker (like physical access to certain ICS components). This work is specific to 
water treatment plants, so the authors define additional security goals of the model to include physical preventions like over/under flow of tanks, increases/decreases in pressure, and arbitrary changes of components (opening and closing of a valve).

The authors demonstrated the extended model on a water treatment testbed with two specific attacker models shown in Table 7. The Insider profile represents an employee or contractor with full physical and virtual access to the control system. The Cybercriminal represents a typical cyber miscreant from the dark web with little knowledge of the system and no physical access. The demonstration revealed attack traces that compromised the security goals stated earlier. A practical analysis was later performed, with real people attacking the water treatment testbed roleplaying as either insiders or cybercriminals. The formal analysis was able to detect 7 of the 8 attacks performed in the practical analysis.

Table 7: Comparison of attacker profiles, from [118]

\begin{tabular}{|c|c|c|c|c|c|c|c|}
\hline Profile & 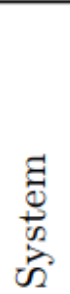 & 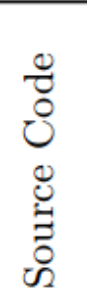 & 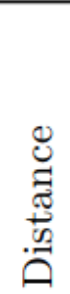 & 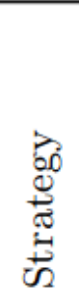 & 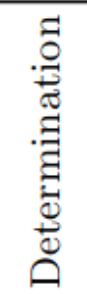 & 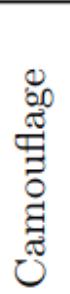 & $\stackrel{g}{4}$ \\
\hline $\begin{array}{l}\text { Insider } \\
\text { Cybercriminal }\end{array}$ & $\bullet$ & $\bullet$ & $\stackrel{\bullet}{\circ}$ & $\bullet$ & $\bullet$ & $\bullet$ & $\begin{array}{l}\text { physical } \\
\text { virtual }\end{array}$ \\
\hline
\end{tabular}

2.5.3 Anomaly detection in cyber-physical systems: A formal methods approach In 2014, Jones, Kong, and Belta presented a method of detecting anomalies in cyber-physical systems through an artificial-intelligence-generated formal specification [120]. Anomaly detection is a common practice in traditional cyber security spaces, and tries to erase the problem of signature detection where the detection system would need 
to know exactly what an attack looks like in order to detect it. Instead, the anomaly detection software knows the expected behavior of the system and can flag activity that does not conform to expectation. The authors note that cyber-physical systems are designed with the assumption that the design team has perfect knowledge of the system. While this may be true in the design and implementation phases, this assumption breaks down as the system gets more complex and human actors act like humans. Designs also seldom account for human actors behaving maliciously, and such behavior is intentionally hard to predict.

Jones et al. make use of a subset of signal temporal logic (STL) [73] to create formulae that describes how a cyber-physical system should behave. Creating such formulae to describe system behaviors is a difficult problem, even when correct models of the system are available, so building a set of formulae through monitoring normal use of the system would be beneficial. Monitoring and analyzing all the variables in a cyberphysical system creates high dimensional datasets with many records, and such datasets are the domain of artificial intelligences. The authors created an unsupervised learning algorithm that can produce formulae describing expected activities of the system. While the specifics of the algorithm is beyond the scope of this survey, its machine-checkable and human-readable outputs are significant.

\subsubsection{Formal modelling and analysis of DNP3 secure authentication}

The Distributed Network Protocol, Version 3 (DNP3) [23], [24], [26], [121] is a widely-used and rigorously specified SCADA networking protocol. DNP3 was originally designed to be reliable, but had no mechanisms for security and many attacks have been demonstrated or theorized in [106]. DNP3-SA, or Secure Authentication, attempts to add 
encryption and authentication through inclusion of cryptographic algorithms [9], [42]. Specifically, DNP3-SA uses two methods for adding security: non-aggressive challengeresponse to force a client to authenticate itself upon receiving a critical instruction, and aggressive mode that allows the client to bypass the challenge-response pattern by proving it successfully completed the previous challenge-response. However, the specification for DNP3-SA is informal and ambiguous, leading to difficulty in both implementing and analyzing the specification for weaknesses.

Amoah, Camptepe, and Foo presented a formalized specification of DNP3-SA and a formal security analysis of the specification [42]. This research used Coloured Petri Nets (CPN), a formal modeling language for discrete events [122]. CPN allows building a master and slave, then building the protocol instructions and creating a state space of possible behaviors through simulation. The authors also formalized the desired security property, stated informally as: The slave is able to authenticate the master station if the master station is able to produce a valid HMAC tag. The state space can then be traversed to find states where the property is violated.

As might be expected from this research's inclusion in the survey, DNP3-SA was found to be vulnerable. The security property was violated and the trace produced a previously-unknown vulnerability. The specific vulnerability results from the relation between non-aggressive mode and aggressive mode. DNP3-SA messages are sent in clear text and can be manipulated. An attacker can break the protocol by intercepting the challenge message and incrementing the sequence number to force an authentication failure. Because the sequence numbers would then increment, the "old" response from the master can then be used in aggressive mode to carry out whatever command suits the 
attacker's fancy. Amoah et al. also propose a solution and proof the solution removed the vulnerability, but at time of writing this solution has not been added to the standard.

\subsubsection{Attack taxonomies for the Modbus protocols}

Modbus is a relatively simple and widely-used SCADA communication protocol developed in the 1970s [22], [123], [124]. It has a call-and-response structure and no security considerations whatsoever [21], [125]. Modbus has two variants: Modbus Serial where in a master communicates with slaves over a serial line, and Modbus TCP where a set of masters can communicate with possibly overlapping sets of slaves. Huitsing et al. analyzed both Modbus specifications and developed a taxonomy of attacks for each [105], finding 20 attacks for Modbus serial and 28 attacks for Modbus TCP.

The authors identified four threat categories for their taxonomies: Interception, Interruption, Modification, and Fabrication. The attack vectors for each Modbus variant included the master devices, the slave devices, and the communication link connecting them, and required materials are simple a network sniffer and some device to introduce fabricated messages to the network. A sample of the attacks is listed in Table 8 .

Table 8: Sample of Possible Attacks against Modbus

\section{Modbus Serial}

Diagnostic register reset

Remote restart

Slave reconnaissance

Broadcast message spoofing*

Baseline response delay*

Direct slave control*

Modbus network scanning*

Passive reconnaissance*

Response delay*

Man-in-the-middle*

\section{Modbus TCP}

Broadcast Message Spoofing*

Baseline Response Delay*

Direct Slave Control*

Modbus Network Scanning*

Passive Reconnaissance*

Response Delay*

Man-in-the-Middle*

Irregular TCP Framing

TCP FIN Flood

TCP Pool Exhaustion

TCP RST Flood

*Attack affects both protocols. 


\subsection{Summary}

This chapter presents a direction and purpose of formal methods and security research efforts generally and specifically for industrial control systems. A lot of research effort has been put into verifying the cryptographic primitives and systems to ensure secrecy, authentication, and integrity can be strongly preserved as in sections 2.3.1, 2.3.2, and 2.3.3. Section 2.4 identifies research into verification of operating systems from general, exceedingly abstract models to verified implementations with dozens of verified security properties. Industrial control systems-specific formal work can be seen in 2.5 , with a distinct focus on protocols, vulnerability hunting, and modeling entire systems. The original research found in this thesis recognizes previous work at the system level and control network protocol level and fills in a gap present at the control network device level. Moreover, previous work has a focus on new control systems as they are deployed, and limited effect on legacy control systems currently in use. This thesis fills the support gap in which legacy systems currently reside. The remainder of this document presents a novel workflow for moving from a verified model to implementation in seL4 and describes a verified specification of a security preprocessor for adding security properties to existing control systems using legacy protocols. 


\section{CHAPTER III}

\section{HIGH ASSURANCE CYBER-SECURITY DEVICES FOR INDUSTRIAL CONTROL SYSTEMS USING TLA+ AND SEL4}

\subsection{Introduction}

While high assurance of any device that could affect a factory floor or distributed control network has always been a goal, the focus has usually been on safety and durability rather than security. Additionally, the methods for achieving high assurance are typically exhaustive testing at the application level and hardware level of the device, leaving out the operating system and any other unnecessary services that may be running [126]. Sixnet RTUs for instance run their application on top of Linux, Allen-Bradley's PLC5 runs on top of Microware OS-9, and a few others run on top of VxWorks [127]. These devices are reliable but possess no proven security or safety properties. As demonstrated with a Sixnet Remote Terminal Unit (RTU) in [128], the operating system could potentially violate environmental assumptions made by the designers during development and present new vulnerabilities.

This thesis presents a novel approach for verifying cyber security relevant properties for control system devices. Using a microkernel that isolates application components, an architecture can be developed that can be treated as a distributed system from a security perspective. Different pieces of code with critical responsibilities can be isolated from one another, their interactions with each other can be strictly controlled, and proper separation of duties can be established in a similar fashion to the separation 
kernel described by John Rushby in 1981 [129]. Reasoning about distributed, concurrent systems can be accomplished with formally specifying the system and model checking the specification. This presents a potential development process for creating fully verified designs, if not fully verified implementations. The development stages in Figure 10 show how to proceed from an idea for an embedded device with high assurance requirements to an implementation ready for deployment on a microkernel capable of isolating components. Development begins with informal discussions of requirements and desired properties of the device, with a special emphasis on separating critical duties into isolated components. These initial concepts are then formalized with a modeling language. Often, the process of formalizing the specification and security properties uncover design flaws before the verification step takes place.

The third step is to structure the architecture of the microkernel to match the formal model. This definition includes any special hardware interface capabilities that the component might need such as network interfaces and storage. The communication between components must also be defined. If the formal model was designed with an appropriate level of abstraction, the microkernel architecture should be simple to implement. The formal specification gives precise documentation for exactly what connections and capabilities each component needs.

The process described in Figure 10 can be applied to any high-assurance embedded device. Isolated moving parts allows for small pieces of larger systems to be verified independently. Smaller, isolated pieces also facilitate code reuse and abstraction, simplifying the design and implementation steps of future projects. With regard to industrial control systems, this process can be applied to the development of any 
embedded device that typically operate in the network such as the RTUs, PLCs, modems, intelligent electronic devices (IEDs), and multiplexers. This process would probably not work well for the more complex hosts on a control network such as data historians and operator workstations that often run a full commercial operating system like Windows. This proposed work is specifically concerning the security of these control systems rather than their operation, so the following section describes a security preprocessor embedded device intended for use in control systems.

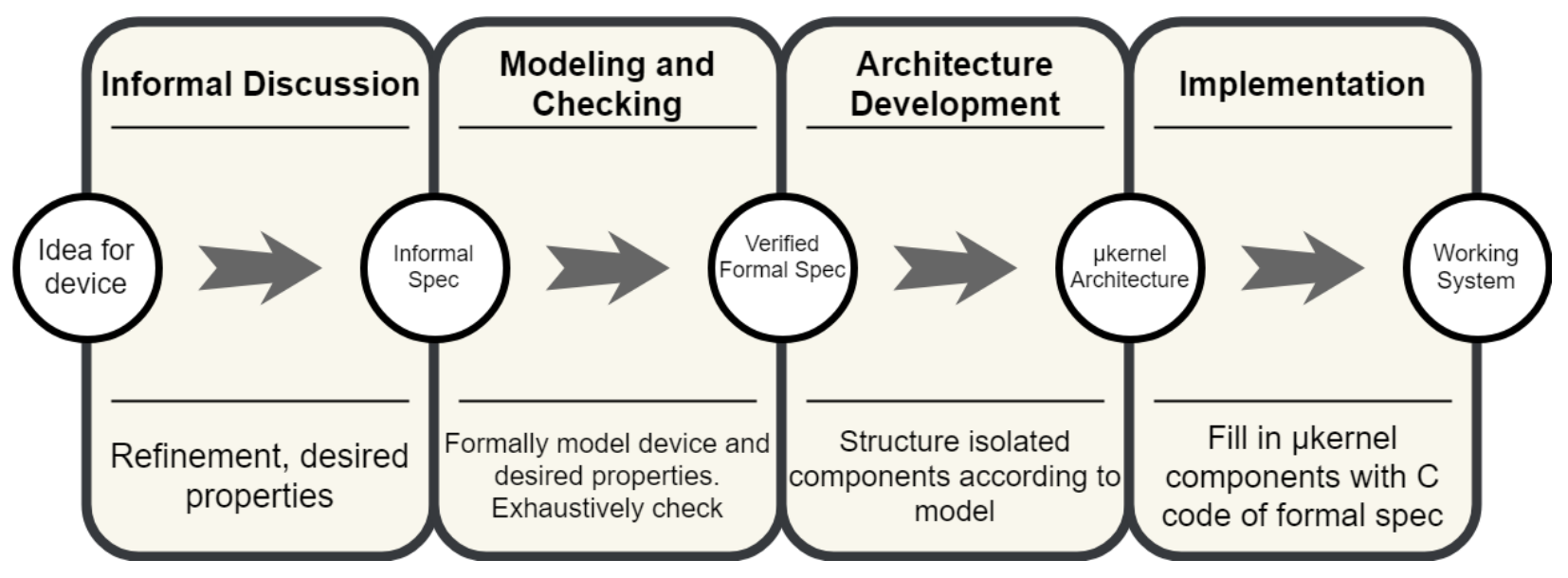

Figure 10: Development steps for verifying embedded control system devices.

\subsection{Industrial Control Systems (ICS)}

An industrial control system (ICS) network can be spread over a large geographic distance. While communication between nodes within a single factory floor might be easy and reliable, reaching substations and water towers across a metropolitan area is more difficult. ICS operators utilize a variety of communication media to reach these field sites, with differing levels of inherent privacy and resistance to tampering. The topology shown in Figure 11 is typical of a water treatment facility control network. The control center is where the engineers and operators will spend most of their time. The 
control center might contain a server to record and archive all activity on the process network called a data historian, the engineer workstations, SCADA servers or programmable logic controllers (PLCs), and a multiplexer for communicating outside. The water towers are located away from the command center, and might be reached via telephone line, leased line, radio towers, cellular networks, or in the worst-case scenario the open internet. The field sites usually contain a remote terminal unit (RTU) for interacting with the physical processes at the field site and a modem to communicate with the command center. ICS network protocols are notoriously lax with respect to security mechanisms as seen in the attack taxonomies in [105], [106]. The stretch of network infrastructure between the field sites and control center presents an attack vector as long stretches are difficult to physically protect. Messages traversing these stretches are vulnerable to tampering. An attacker could even introduce new, fraudulent messages to the network. Control systems developed before security was a significant concern would have little recourse against this sort of attack.

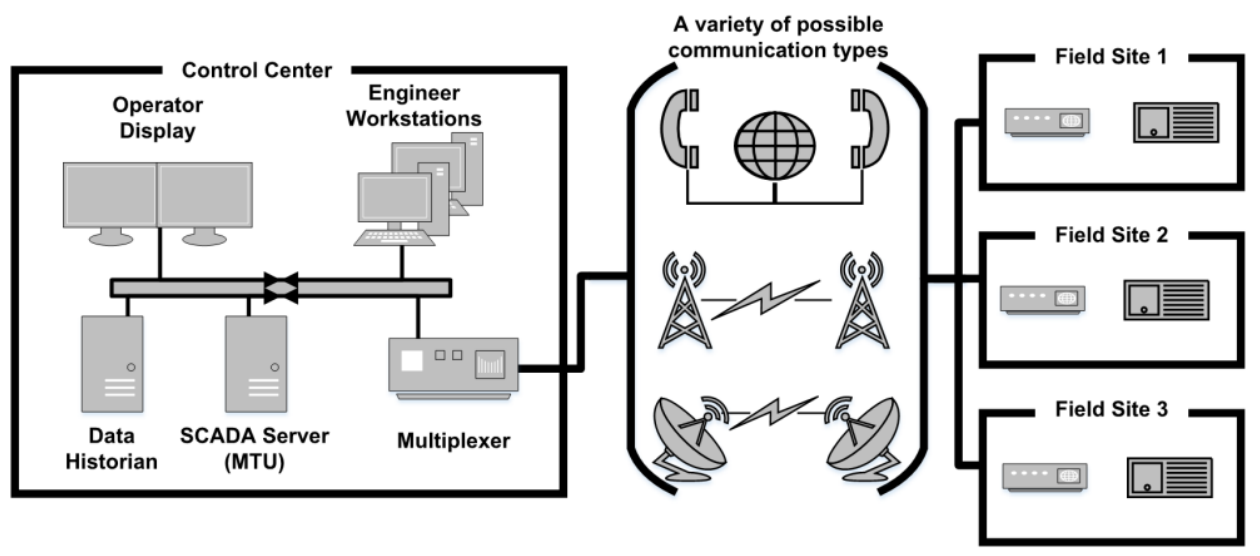

Figure 11: A typical ICS network topology, adapted from [130] 
The communication between the field sites and the command center is the focus of the work presented in this chapter. Adding security to the communications from field site to command center will remove the burden of trusting the networks the messages might traverse. Adding security to an ICS network is not as simple as doing so for traditional corporate networks - timing requirements are stricter, downtime is less tolerated, and additional complexity usually means more points of failure. This chapter discusses the use of the seL4 microkernel and the TLA+ specification language in attempts to create a highly reliable embedded system. Additionally, this chapter describes previous work on a bump-in-the-wire ${ }^{2}$ security preprocessor that could potentially see its assurance level benefit from the proposed development process.

\subsection{TLA+}

Specifying and exhaustively exploring the state space of a distributed and concurrent system is more difficult than in a sequential system. The order of actions taken in the separate pieces of executing code is not defined, and one piece can affect the environment of another, changing the behavior of both. Temporal Logic of Actions was designed specifically for concurrent systems [63]. TLA+ is a formal specification language with semantics that support temporal logic of actions and modeling complex distributed systems [64], [101], [131]. Understanding the semantics of TLA+ will help the reader understand how it can be applied and used to check for security and safety properties. This section presents a simple example of a distributed decision-making

${ }^{2}$ Bump-in-the-wire means the system would be put on the network in between two components and act without either component noticing a difference. 
system: multiple database resource managers trying to agree on whether to commit to a transaction.

In the TCommit algorithm, described in [132] and displayed in Equation (5), is a process for safely executing a database transaction. The transaction is performed by a set of resource managers acting concurrently. The transaction can either commit or abort. The resource managers can either be working, prepared to decide, committed, or aborted. The desired properties for the system are:

1 The transaction can only commit if every resource manager is prepared to commit.

2 If a single resource manager aborts, the transaction must be aborted.

3 All resource managers must agree on whether the transaction committed or aborted.

The first line the TCommit specification defines is a set of resource managers $R M$.

The second line defines an array rmState that is indexed by the set of resource managers. TCTуреOK defines an invariant property. TCTуреOK is true if the state of any given resource manager is an element of the set "working, prepared, committed, aborted". If a resource manager finds itself is any other state, this property is violated. The expression rmState $[r]$ will give the state of resource manager $r$. TCInit describes the initial state of the system. In the initial state, rmState is the array indexed by $R M$ such that every resource manager $r$ is in the "working" state. The statement canCommit is true when every resource manager is in the prepared or committed state. The statement notCommitted is true if no resource manager has decided to commit. Following these definitions are the actions that a resource manager can take. Prepare can occur when resource manager $\mathrm{rm}$ is in the working state. The next state is one in which $\mathrm{rm}$ is prepared and the other resources managers are the same state they were in the previous state. 
Decide can occur when a given resource manager $\mathrm{rm}$ is in the prepared state and canCommit is true OR when $\mathrm{rm}$ is in the working or prepared state and no other resource manager has committed, leading to an abort.

The important line in this simple specification is TCConsistent. This invariant property is true if no two resource managers are in the committed and aborted states at the same time. When this model is used as input to the TLC model checker, every generated state will be checked for conformance to TCConsistent. If TLC finds no state which violates the property, then work is complete.

TCInit $\triangleq$ rmState $=[\mathrm{rm} \in \mathrm{R} \mapsto$ working $]$

TCType $\mathrm{OK} \triangleq \mathrm{rmState} \in[\mathrm{RM} \rightarrow$ \{working, prepared, committed, aborted $\}$ canCommit $\triangleq \forall \mathrm{rm} \in \mathrm{RM}: \mathrm{rmState}[\mathrm{rm}] \in$ \{prepared, committed notCommitted $\triangleq \forall \mathrm{rm} \in \mathrm{RM}: \mathrm{rmState}[\mathrm{rm}]$ \# committed

Prepare $(\mathrm{rm}) \triangleq \wedge$ rmState $[\mathrm{rm}]=$ working

$\wedge$ rmState $^{\prime}=[\mathrm{rmState}$ EXCEPT $![\mathrm{rm}]=$ prepared $]$

$\operatorname{Decide}(\mathrm{rm}) \triangleq \vee \wedge$ rmState $[\mathrm{rm}]=$ prepared

$\wedge$ canCommit

$\wedge$ rmState $^{\prime}=[\mathrm{rmState}$ EXCEPT $![\mathrm{rm}]=$ committed $]$

$\vee \wedge$ rmState $[\mathrm{rm}] \in\{$ working, prepared $\}$

$\wedge$ notCommitted

$\wedge$ rmState ${ }^{\prime}=[$ rmState EXCEPT $![\mathrm{rm}]=$ aborted $]$

$\mathrm{TCNext} \triangleq \exists \mathrm{rm} \in \mathrm{RM}: \operatorname{Prepare}(\mathrm{rm}) \wedge \operatorname{Decide}(\mathrm{rm})$

TCSpec $\triangleq$ TCInit $\wedge$ [][TCNext]_rmState

TCConsistent $\triangleq \forall \mathrm{rm} 1, \mathrm{rm} 2 \in \mathrm{RM}: \neg \wedge \mathrm{rmState}[\mathrm{rm} 1]=$ "aborted"

$\wedge$ rmState[rm2] $=$ committed

\section{4 seL4 and CAmkES}

The seL4 microkernel has been fully verified from design to implementation to provide a high level of assurance [109], [110], [133], [134]. seL4 was the evolution from the OKL4 family of microkernels developed to reduce the size of a microkernel to the point where a guarantee of bug-free code could be realized. seL4 provides a verifiedcorrect ability to logically separate processes and implement highly specified channels of communications between components within the architecture. If a cell in the kernel is 
compromised, it can be shown that the other cells still hold true to their desired security properties. This allows an abstract implementation of Rushby's separation kernel for reducing large security kernels into smaller, more easily provable, components mimicking a distributed system. seL4 was developed using Haskell for prototyping and Isabelle/HOL for the heavy proof work. It comprises 10,000 lines of verified C code requiring 18 person-years of development time as of 2018 [133].

Component Architecture for microkernel-based Embedded Systems (CAmkES) is a component platform designed to address the increasing complexity and unreliability of embedded systems through facilitating a modular design of system services [135], [136]. The CAmkES framework provides a language for describing components, component interfaces, and shared memory. During the build process, each component description is translated into scaffolding and glue code that houses the source code (usually in C) for the service that the component provides to create a bootable system image. This automatically generated glue code is responsible for initializing the component at boot, running threads, and managing the component's resources, as well as facilitating the communication between components. Full verification of the generated glue code is a work in progress, but the remote procedure call (RPC) portion that allows one component to utilize the service of another has been verified to behave as though the service were provided by the originating component itself [137].

CAmkES is the recommended tool for creating native seL4 applications and comes integrated into the seL4 build system. Only a single CAmkES application can be running at a time. Applications built with CAmkES are static, meaning all the specified components and connections are created at boot time. No components, connections, or 
interfaces can be created or destroyed during runtime. Additionally, components have a fixed amount of memory available, defined during the design. The philosophy behind a static application is to reduce the complexity of the verification efforts and allow guarantees to be made about the system's operation. The target for CAmkES, embedded systems, is often static anyway. A device designed for control systems would also be static, as it would likely be in the field for a long time with a single responsibility and no need for feature changes beyond the initial deployment.

\subsection{Application of Verified Systems for Control Systems Security}

The guarantees offered by the seL4 microkernel can allow a higher level of assurance to be achieved than with previous high assurance microkernels. The verification work of the microkernel paves a path to fully verified software stacks. The CAmkES architecture language and support provides a framework for building native applications in small and verifiable chunks. The seL4 microkernel allows the development of an embedded system that mimics a distributed system, so TLA+ could be a valuable tool in reasoning on these designs. This presents a potential development process for creating fully verified designs, if not fully verified implementations. The development stages described in previously in Figure 10 can be expanded, with Figure 12 showing in more detail how to proceed from an idea for an embedded device with high assurance requirements to an seL4-based implementation ready for deployment using these techniques. The nature of CAmkES must inform the level of abstraction used in the formal specification. For example: how the components communicate could potentially be left out of the specification, but what the components are saying to each other should be explicitly specified to aid in the next stage of development. With the formal 
specification and formal properties, the TLC Model Checker is used to ensure the specification accurately models the design, with each component behaving correctly and the interactions between components precisely understood.

The third step is to define each component formally described in TLA+ within CAmkES. This definition includes any special hardware interface capabilities that the component might need such as network interfaces and storage. The communication between components, handled with remote procedure calls (RPCs) in sel4, must also be defined. Each component provides interfaces that other components can use to access services. These interfaces and their structure (the calls, the parameters, and return values) are statically defined before compilation and do not change after boot. Finally, the RPC connections must be defined. A connection must be defined for each interface a component might use during operation. If the TLA+ model was designed with an appropriate level of abstraction, the CAmkES definitions should be trivial to implement. The formal specification in TLA+ give precise documentation for exactly what connections and capabilities each CAmkES component needs.

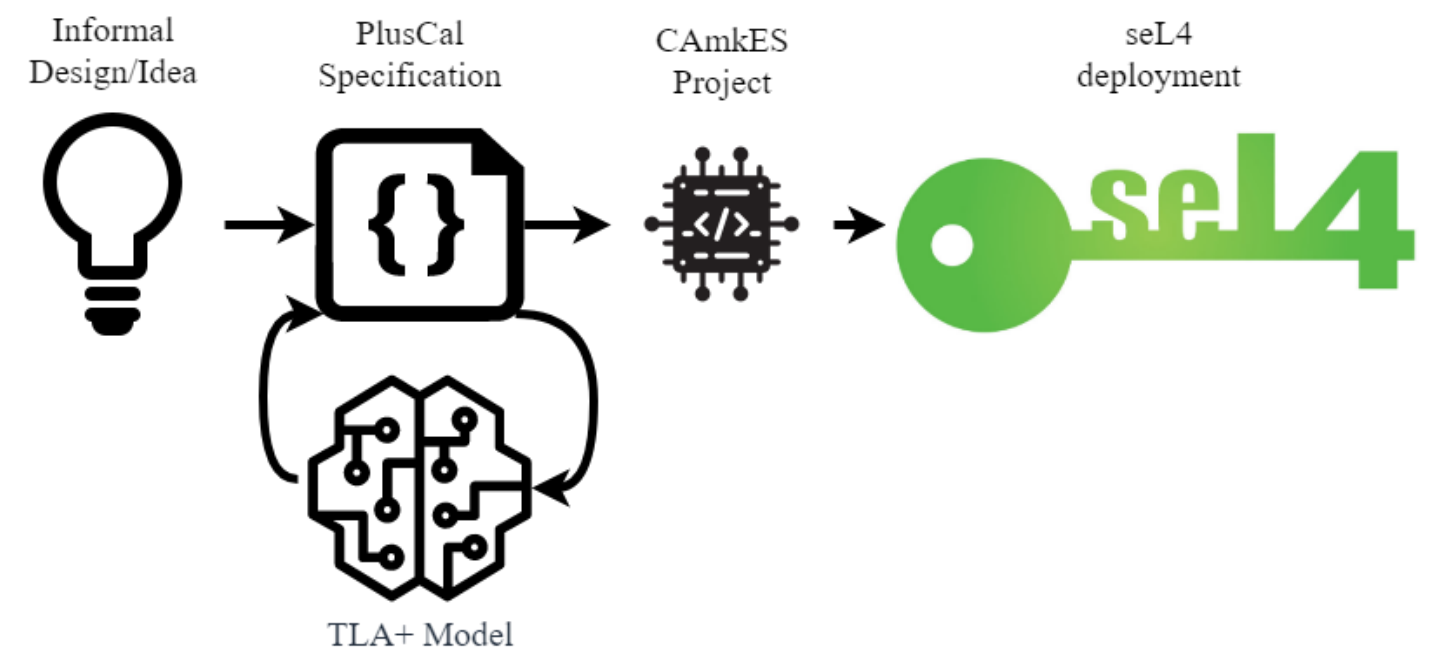

Figure 12: Development steps for verifying seL4 designs using TLA+. 


\subsection{Translation of TLA+ and PlusCal into CAmkES}

Formally verifying a system design with this method involves specifying the system in PlusCal, translating the PlusCal to TLA+ for model checking, then translating the PlusCal to a CAmkES project that can be deployed on seL4. CAmkES provides primitives (Table 9) to designers that come with security and safety guarantees. These primitives can be modeled in TLA+ and used in building specifications. With careful modeling, these specifications can be directly translated from PlusCal to CAmkES.

Table 9: CAmkES primitives

\begin{tabular}{|c|c|}
\hline CAmkES pr & Description \\
\hline Component & $\begin{array}{l}\text { A logical grouping of code and resources. Code within } \\
\text { components has access to all the memory that was assigned to the } \\
\text { component, but not other components. }\end{array}$ \\
\hline Con & A method of communicating between two components \\
\hline Interface & The definitions of function calls that occur over connections \\
\hline
\end{tabular}

A component is modeled in PlusCal using a process. Processes in PlusCal are like processes in classical computer science. They have their own local variables just as a process would have its own address space, but unlike classical processes they can read and write global variables that could potentially affect other PlusCal processes. Because of these differences, modeling a classical process in PlusCal requires care from the programmer to limit the reach of a PlusCal process to reading and writing specific global variables in a controlled manor. In translation, a new component is created for each Process keyword found in the PlusCal. Each component requires a new directory with a camkes file and a src directory that contains the $\mathrm{C}$ code implementation. An example CAmkES hierarchy is illustrated in Figure 13. 


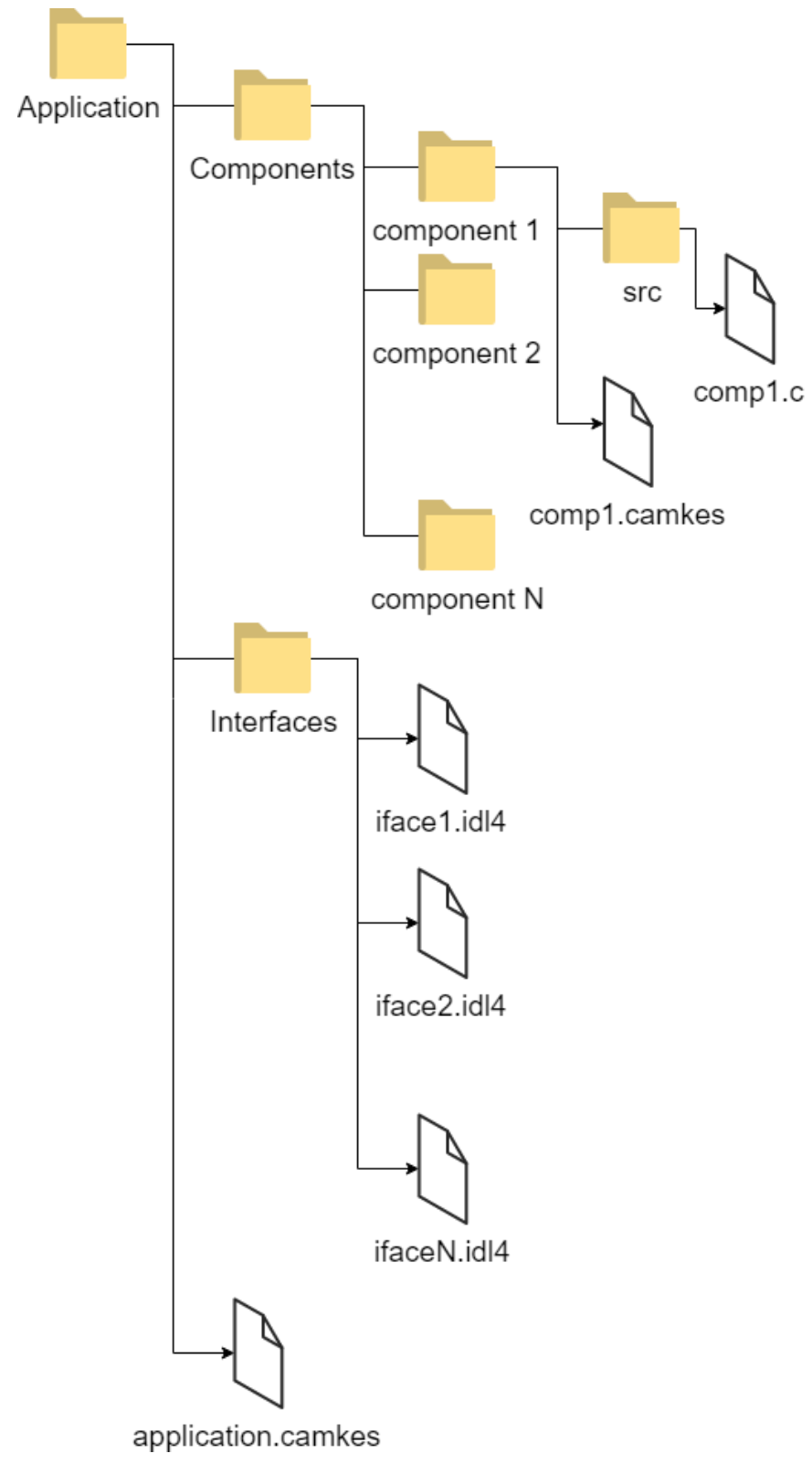

Figure 13: CAmkES directory setup for an example application. Each component has its own directory housed within the "Components" directory. Each component directory has a camkes file and a src folder containing $\mathrm{C}$ code.

Modeling communication between components is accomplished using a macro called Send. Send contains the needed information to construct both an interface and a connection. Send is given in Figure 14. It takes as parameters a destination queue (in practice, this can be thought of as a destination process) and a message. When translating to a connection in CAmkES, an seLARPCCall is declared from the sending process to the 
receiving process. Figure 15 shows Send calls that originate from Modtx and forward messages to Crypto and Modchk, along with the corresponding connections created in the CAmkES project.

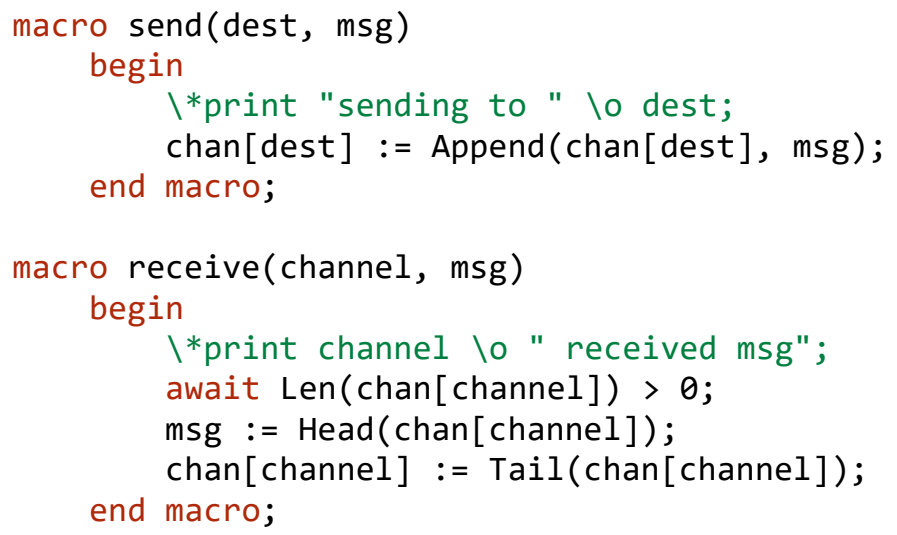

Figure 14: PlusCal definitions for Send and Receive macros

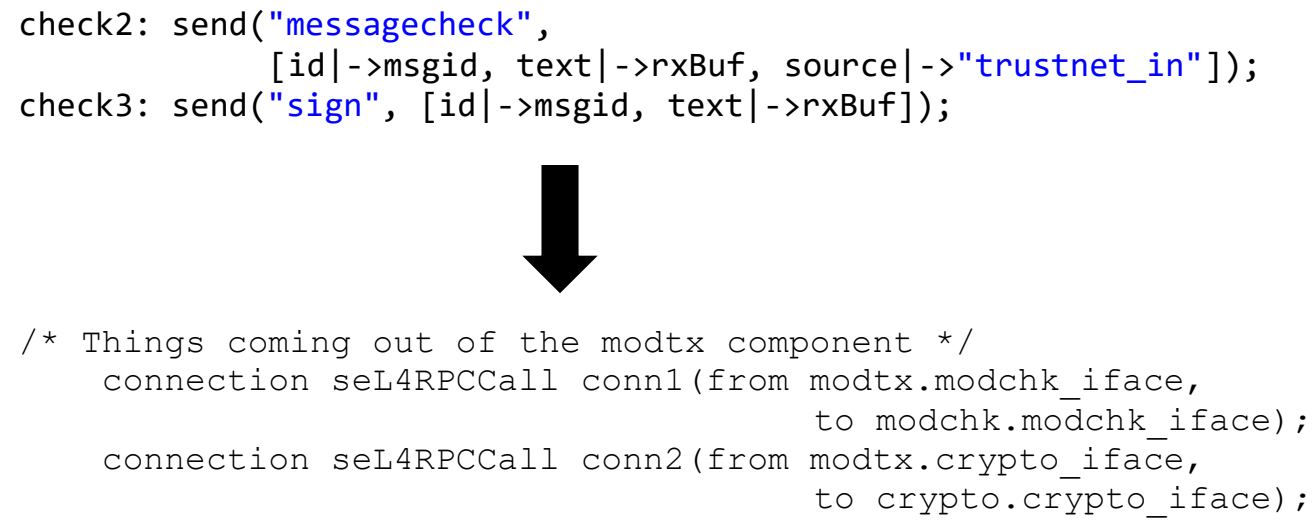

Figure 15: Send macros in TLA+ and their translations into CAmkES connections. Declarations of seL4RPCCall connections from the Modtx component to the protocol checking component (conn1) and the crypto component (conn2)

Finally, translating Send macros into interfaces can be tricky, as TLA+ is an untyped specification language and CAmkES defines interfaces using the strongly typed C programming language. There have been efforts to add a refinement type system to 
TLA + [138], but those were not used here. Instead, invariants were used to ensure variables conformed to their proper encodings within TLA+, and the types were translated manually into C. The Send macro example from Figure 15 is refreshed in Figure 16 along with its translation into an interface.

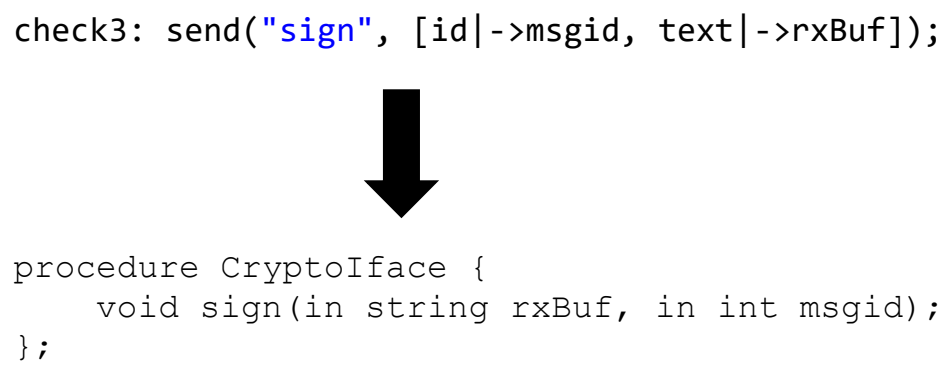

Figure 16: A Send macro in TLA+ and its translation to a CAmkES interface

Both components involved in the operation see their .camkes files modified to reflect that the receiving process provides the interface and the sending process consumes the interface. These modifications are seen in Figure 17.

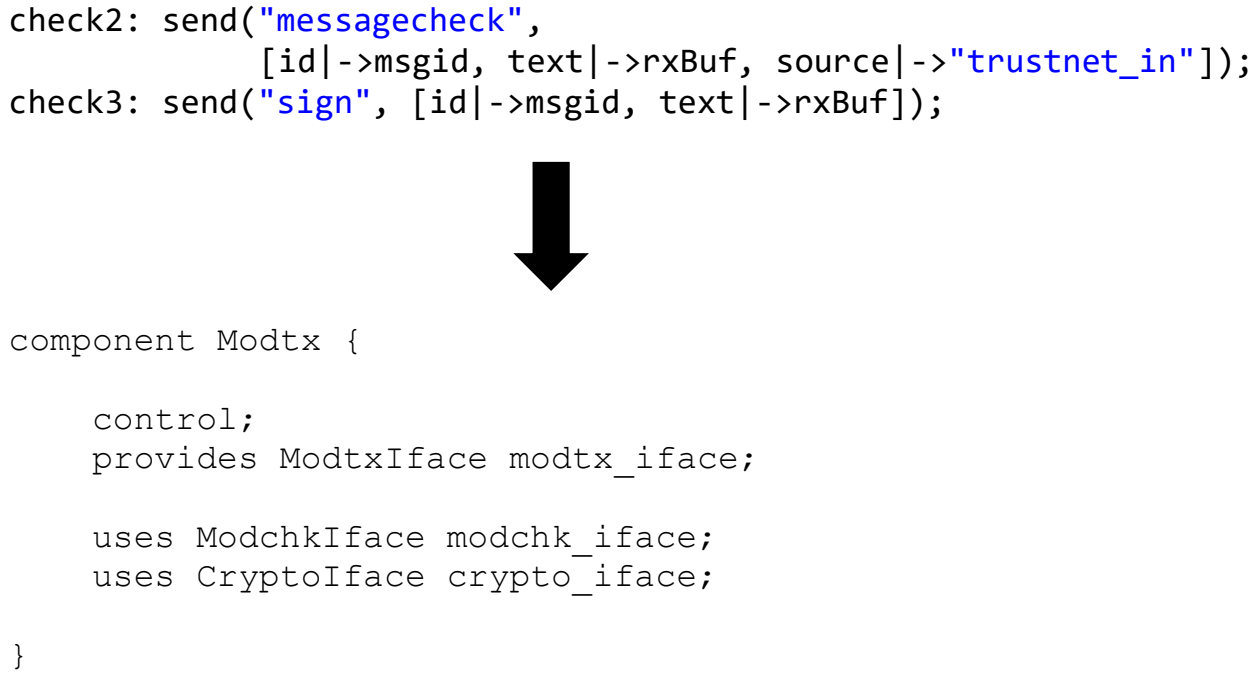

Figure 17: Translation of a PlusCal send macro to a CAmkES component definition

The steps for translating from TLA+ (PlusCal specifically) to CAmkES can be written as follows: 
1. Using the Send and Receive macros for inter-process communication, specify a system with multiple processes that are well behaved (i.e. they do not modify global variables other than through Send and Receive).

2. Create a CAmkES project with an empty directory structure

3. For each process:

a. Create a subdirectory structure with name matching the process label

b. Create a .idl4 interface file in the interfaces directory with name matching the process label

4. For each process:

a. For each Send macro in the process:

i. Create a new interface declaration (if one does not already exist with this name and parameters) in the sending process's idl4 file

ii. Within this interface declaration, create a $\mathrm{C}$ function prototype with:

1. return type void

2. parameters matching the field names in the second argument of the Send macro and types as specified in the invariants

iii. Within the .camkes file for the sending component

1. Insert "uses *receiving_component_interface*"

iv. Within the .camkes file for the receiving component

1. Insert "provides *receiving_component_interface*"

v. Create a new seL4RPCCall connection from sending process to receiving interface in the root camkes file

The final step is to fill the components with $\mathrm{C}$ code that implements the behavior specified in the TLA+ model. This cannot be automated in this context as TLA+ is designed for reasoning on the what tasks a system accomplishes, not how the system accomplishes those tasks. TLA+ specifications provide good guidance on how to implement the $\mathrm{C}$ code within the components, but this is a manual task.

\subsection{Security Preprocessor as Previously Designed}

In 2012, Hieb, Graham, Schreiver, and Moss presented a design and prototype for a Field Device Secuity Preprocessor (FD-SPP) to create and protect a perimeter around field devices located at remote stations in a SCADA network [130]. The work builds from previous papers describing a security hardened remote terminal unit (RTU) built 
from the ground up with security as a focus [139]. This hardened RTU logically isolates security-critical code such as cryptographic services, access control enforcement, and the policy decision point from each other and from the network interfaces. Feedback on this work led to pulling the security processing steps out of the RTU and into a separate device to minimize impact of installing such features into an existing network. The FDSPP is an embedded control systems security device to which the proposed formal verification techniques could be applied.

The FD-SPP can act as a bump-in-the-wire configuration to allow a simple installation of two devices at either end of a communication line. Installation should be as simple as plugging them in and turning them on - no other devices on either end of the network would need to be bothered. Additionally, the FD-SPP should not add any new attack vectors to the network. The layered security of isolated components and cryptographic mechanisms would reduce the attack surface and make compromising the application very difficult. Figure 18 simplifies a SCADA network to the pieces relevant to this discussion. A Human-Machine Interface (HMI) or a Programmable Logic Controller (PLC) in the control center would communicate through some medium to an RTU at a remote substation. Figure 19 illustrates how a FD-SPP would change the network diagram. One FD-SPP device would need to be placed in the control center right after the HMI, and a second device would need to be place in the substation right before the RTU. Both devices encapsulate messages going out of their respective zones, and both devices decapsulate messages coming into their prospective zones. 


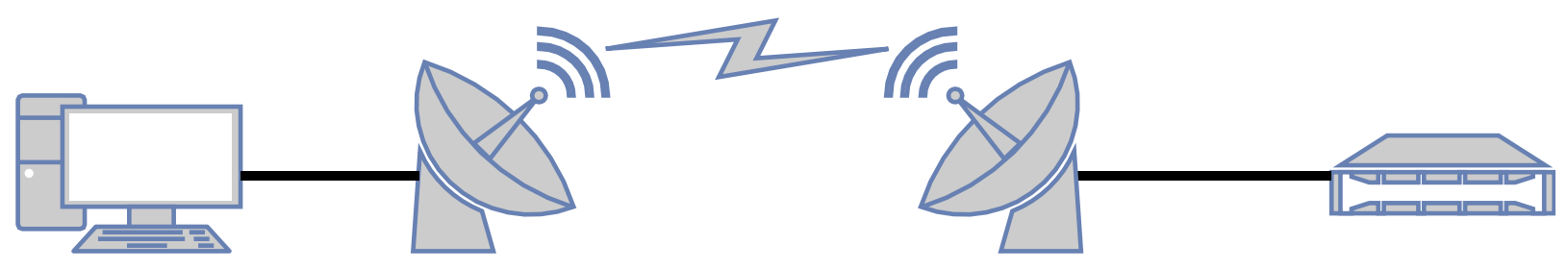

Figure 18: Connection from the control center (left) to RTU (right) on a typical SCADA network

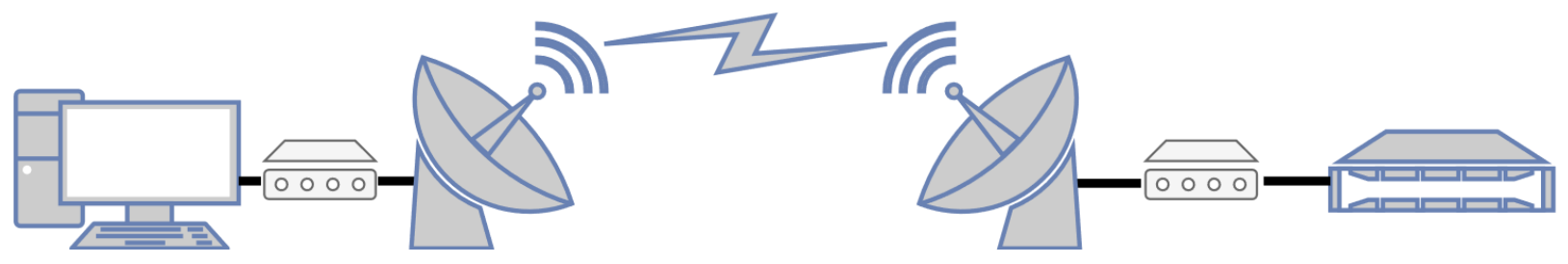

Figure 19: Connection from the control center to RTU with the FD-SPP installed The FD-SPP offered in 2012 was built on top of an unverified but high assurance microkernel. Hieb et al. designed the FD-SPP to operate with three components, or cells, each with their own responsibilities and a limited ability to affect one another. Threads in each cell can communicate with each other normally, but communication between cells in different threads is strictly determined at compile time. The cell configuration can be seen in Figure 20. The three cells act in sequence, with messages moving through all three for processing before reaching the other side. The two outer cells interface with the control network (the prototype utilized a serial port, but this was not necessary). The inner cell acted as the security controller and housed the access control and cryptographic logic. This configuration had the added benefit of protecting the security critical process from the outside world. An attack on this device would need to bypass at least one networking cell before attempting to compromise the security properties. 


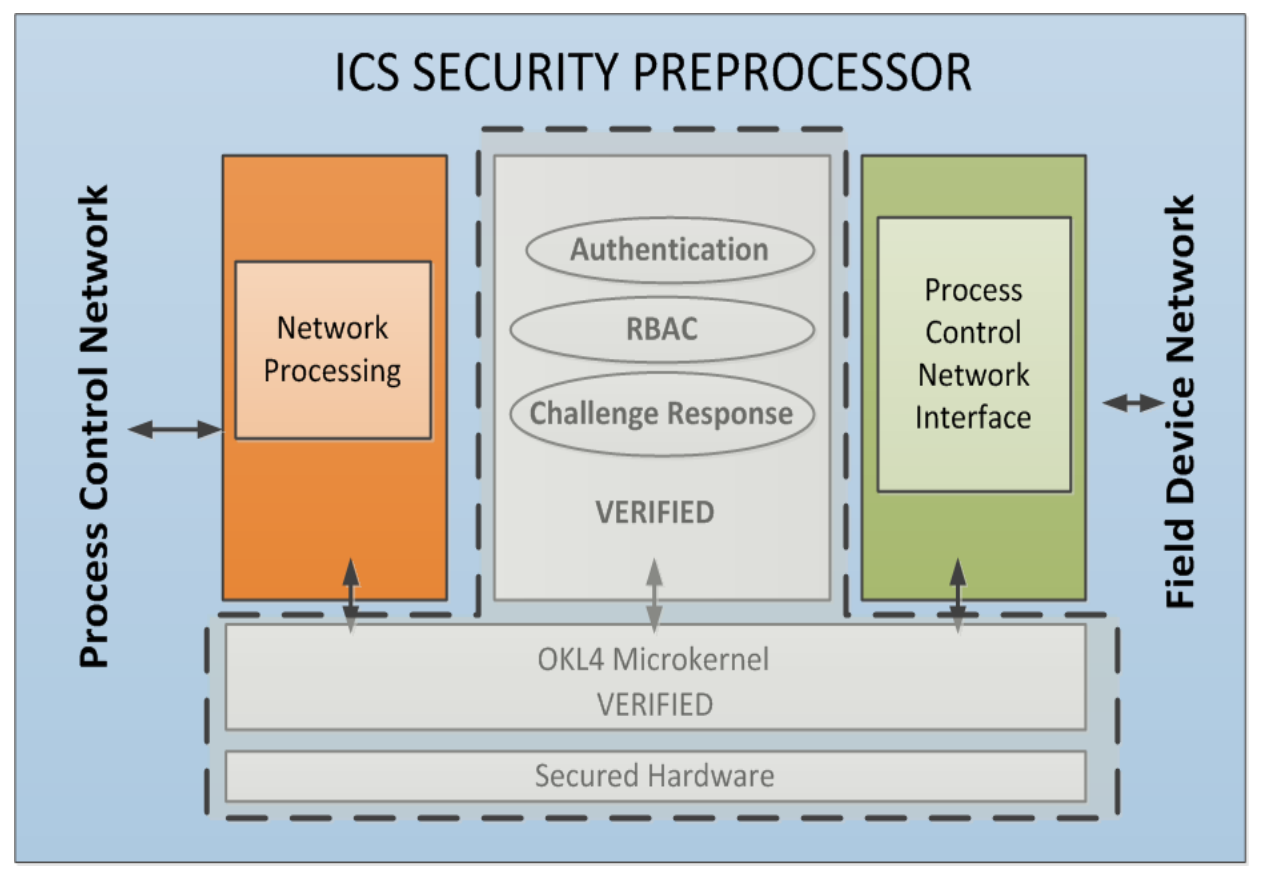

Figure 20: FD-SPP architecture

The FD-SPP as proposed offered access control and authentication capabilities to a Modbus RTU network. Modbus, both Modbus ASCII and Modbus RTU, are missing any sort of security mechanism in their specification. As seen in [29], [105], both mechanisms could be added with overhead small enough to fit the timing constraints in a large portion of SCADA networks. Authentication would be provided through use of a pre-shared key for each user and a challenge-response protocol similar to [42]. The Modbus protocol would be extended to add the required function codes to allow for a challenge-response: a Request code, a Challenge code, and a Response code. A Request message would flow to the field device containing a user ID. The field device would generate a nonce and send it back as a challenge. Finally, the control center device would hash the nonce with the user's secret using SHA-256 and append the result to the original Modbus packet. The field device would also perform the hash with the user's secret and ensure the calculated hash and the response hash are the same. If they are equal, the 
Modbus message can pass through to the RTU. The response from the RTU can pass through the FD-SPP and travel back to the control center without trouble.

Hieb et al. also proposed a Role-Based Access Control (RBAC) mechanism that would operate within the security cell. User roles would be mapped to a set of allowable opcodes and users would be assigned user roles. During the challenge-response process, the user would be authenticated, then checked against an access control matrix. A lowlevel operator might have permission to read the control signals, but an attempt to change control logic would be denied. The difficulty of managing roles and complexity of management led this feature to be discarded in later designs.

The FD-SPP was developed for the OKL4 kernel, a precursor to seL4, and as such is already separated into critical components. The OKL4 kernel limited designers to up to three components per system, but seL4 removes this limitation. The newly designed architecture should add a fourth component to separate the critical protocol checking code from the critical cryptographic code and perhaps have these components work in parallel. Stated very informally, the desired properties of the holistic system include:

1 Isolation between components such that a compromised component cannot affect any others

2 Only proper messages can be allowed to pass through - no malformed messages

3 Only properly formed messages can originate from the device

4 Every message is properly authenticated

5 Every message is protected from tampering

6 All legitimate messages do eventually pass through (except in the case of denial of service)

\subsection{Summary}

Adding security to an existing control systems network requires careful considerations to reduce downtime, reduce added latency, and reduce added failure 
points. A bump-in-the-wire security preprocessor built atop a high-assurance microkernel like seL4 might reduce the impact of added security enough to be palatable to asset owners. This chapter proposed a development cycle for engineering high-assurance embedded systems with formally described and verified security and safety properties. An informal design of an embedded system can be formalized and verified using TLA+. The TLA+ specification can be used to define an architecture in CAmkES. Finally, the components in the CAmkES architecture can be populated with the $\mathrm{C}$ implementations of their algorithms. 


\section{CHAPTER IV}

\section{MODELING A BUMP-IN-THE-WIRE SECURITY PREPROCESSOR}

\subsection{Introduction}

Formally specifying a system must start with choosing the properties the system must possess. Once the properties are chosen, checking those properties informs the design of the specification. The level of abstraction depends on what pieces of the system are relevant to the properties. Choosing which pieces of the system that can be modeled separately depend on how their interactions affect the properties. Metadata like unique message identifiers may need to be included in the model that may not exist in the real system to keep track of the moving parts. The contributions presented in this chapter are the formal specifications written in TLA+ for each piece of a field device security preprocessor that needed to be modeled to capture these desired properties:

1 Isolation between components such that a compromised component cannot affect any others

2 Only proper messages can be allowed to pass through - no malformed messages

3 Only properly formed messages can originate from the device

4 Every message is properly authenticated

5 Every message is protected from tampering

6 All legitimate messages do eventually pass through (except in the case of denial of service)

None of these properties can be directly described in TLA+. Each thread defined in TLA+ in this chapter has subgoals, or smaller properties that can be proven and then used in conjunction to achieve the larger properties. This chapter will first lay out any 
assumptions made when formulating the models, then go into detail on each of the models and their respective properties, and finally explain some novel helper functions and formal definitions that aided in the development.

\subsection{Assumptions}

Every security mechanism, verified or otherwise, works on a set of assumptions that if violated will compromise the guarantees claimed by the mechanism. An assumption can be as simple as "a user's password will only be known by that user" in a corporate network environment. Beyond showing proofs of certain properties, verification forces a designer to rigorously define any assumptions made by the system so they can be addressed in the implementation and risk assessment. When installing a security mechanism into an existing system, care must be taken to ensure all assumptions made by the mechanism are met. Formalizing a design produces a list of assumptions as a by-product that can be included in any documentation to future engineers and operators.

The modeling work presented here works from certain assumptions based on two limitations: the scope of the research, and resources available. The first limitation involves limiting the scope of the research to just the FD-SPP software and architecture. The hardware is assumed to be correct. Techniques for developing reliable hardware have come a long way, but some level of trust is still needed, specifically on hardware that seL4 supports. The second assumption in this category is that the kernel is correct. Use of seL4 allows this assumption to be removed in practice, but the kernel is not modeled in TLA+. The third assumption is that the trusted parts of the control network are behaving properly. The system, and therefore the model, is not acting as an application firewall and deciding whether the valid and authentic Modbus messages are appropriate for the 
control logic. A malicious operator acting on the trusted network could send destructive messages through the system if they are properly formed - the destruction would potentially affect the cyber-physical portion of the network and ideally leave the network nodes unharmed. Finally, this research recognizes the network at large is still vulnerable to denial of service attacks whether from the trusted or untrusted network. Enough malformed messages could be introduced to the untrusted network to prevent valid messages from getting through. The system being modeled does not attempt to address this attack vector.

The second category of assumptions is made to reduce the size of the model and allow a higher level of abstraction to reduce the resource strain on the researcher and the model checker. Only the behavior of each component and system is modeled. How the implementation achieves that behavior is left to future work. The algorithm for each action is modeled, but the code that would run on a live system is assumed to be correct. Secondly, the secret is assumed to be secret. An attacker that holds the secret can break the system. Additionally, each component assumes data from the other components is correct. While proof is given that networking components can only send well-formed messages out to the networks, they are capable of sending invalid messages if they receive invalid messages from the inner components. Finally, the cryptographic algorithms are assumed to hold their claimed properties. This assumption can be eased for HMAC through use of the verified cryptographic code described in [87], and removed through use of CompCert as the compiler. There are currently no proofs that SHA-256 is "secure". The best that can be offered are heuristic arguments. 


\subsection{Formal TLA+ Specifications for Components and Properties}

Formalizing a design in a modeling language like TLA+ requires deciding what is important aspects of a system are important to capture in the model and what aspects are unimportant and can be abstracted to ease the modeling effort. As model checking can quickly run into the state explosion problem, care must be taken to ensure that the system is described sufficiently to be of value, while not getting so detailed as to render checking infeasible. The system described in the remaining sections of this chapter and seen in [140] is modeled component by component, then as a single system. Memory management and communication between the components is abstracted.

\section{Everything should be made as simple as possible, but not simpler. \\ - Albert Einstein}

\subsubsection{Modeling the Trusted Network Component}

The trusted network component is separated into two processes (processes can be thought of as threads), receiving messages from the trusted network and sending messages on the trusted network. The receive process, called trustnet_in, is designed to poll a serial port for input, so its logic is placed within a while loop that executes while there are messages to be processed. The algorithm for this thread is a basic loop, visualized in, that will read a byte at a time from an incoming message and place it into a buffer. After processing is completed, the buffers are cleared and the thread starts again from the top. The more complicated parts come with the desired property that only wellformed Modbus messages reach the inner components. To achieve this, the logic checks each byte of input for ":", indicating the start of a Modbus ASCII message. If a new Modbus message is started before the previous one is finished, the previous message is discarded. Whenever a ":" is received, the contents of the buffer are flushed and the ":" is 
placed at the start of the empty buffer. Similarly, if the buffer is full despite not receiving a complete message, its contents are discarded, and the logic waits for a new ":". Secondly, the logic checks for the termination of Modbus messages. Modbus ASCII messages end with the two-byte sequence "Ir $\backslash n$ ". When this sequence is detected, this thread generates an object containing the message, a generated message ID, and "trustnet_in" and places the object into the signing queue of Crypto. A similar object is created with just the message and ID and placed in the queue of Modchk.

The desired behavior of the Trustnet_in thread is that it accepts only and all wellformed Modbus, forwards only well-formed Modbus, forwards the Modbus to both inner components in the same atomic step, forwards a single message exactly once, and doesn't overflow any buffers. The properties that need to be proven for this thread are seen in Table 10. The formalized property can be seen in the middle and the informal description can be seen on the right. There are several variables and custom operators in the formalized properties that the reader might like to familiarize themselves with in Table 11 before examining the properties themselves. 


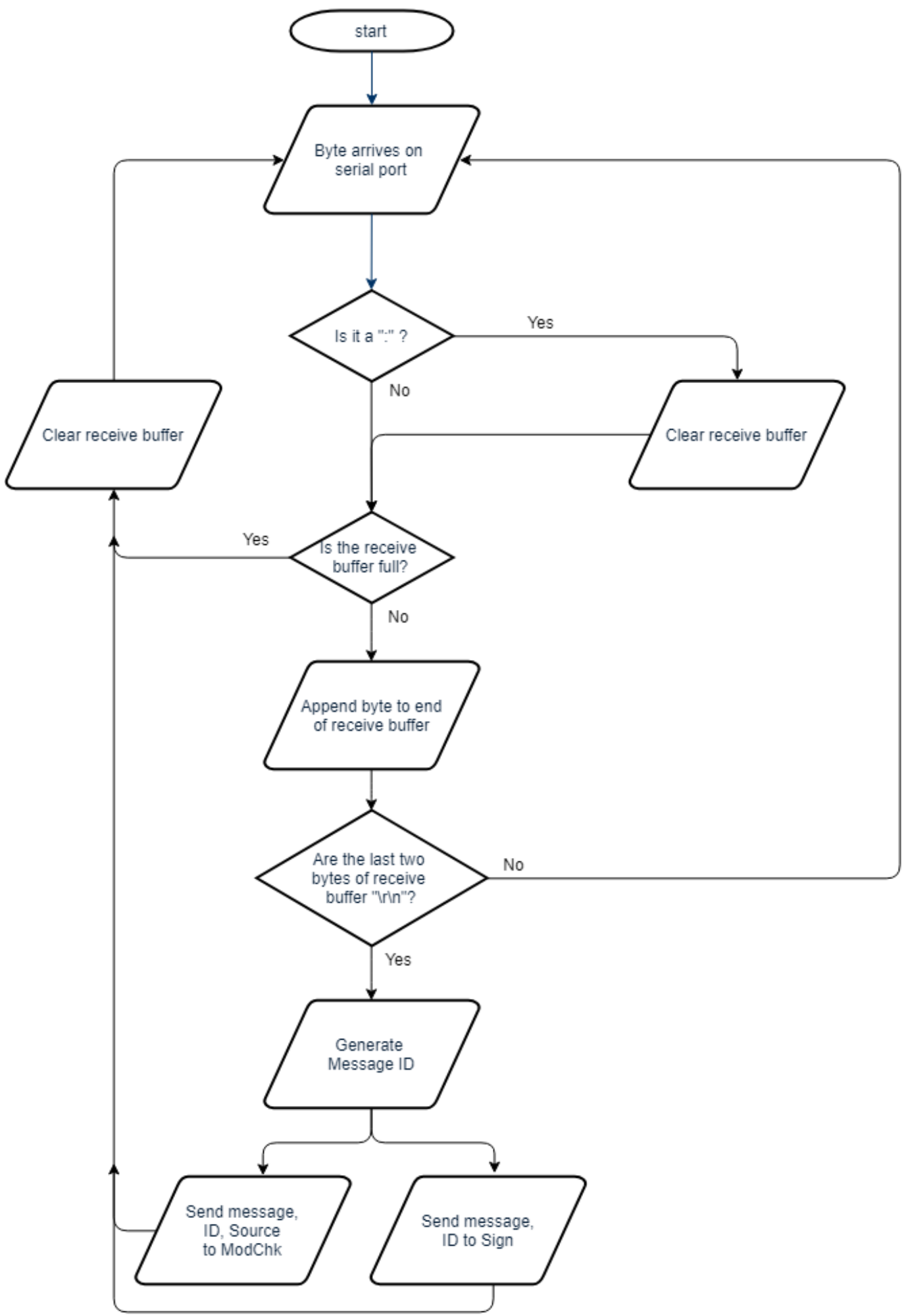

Figure 21: Flowchart for Trustnet_in thread 
Table 10: The desired properties of the Trustnet_in thread

\begin{tabular}{|c|c|c|}
\hline Name & Formalized Property in TLA+ & Informal Property \\
\hline SAFE1 & Len $(r \times B u f) \leq$ MAXMODBUSSIZE & $\begin{array}{l}\text { receive buffer never } \\
\text { overflows }\end{array}$ \\
\hline SAFE2 & $\begin{array}{c}\wedge \forall x \in \text { Range }(\text { signBuffer }): \text { Len }(x . \text { text }) \leq \text { MAXMODBUSSIZE } \\
\wedge \forall x \in \text { Range }(\text { modchkBuffer }): \text { Len }(x . \text { text }) \\
\leq \text { MAXMODBUSSIZE }\end{array}$ & $\begin{array}{l}\text { sending buffer never } \\
\text { overflows }\end{array}$ \\
\hline SAFE3 & Len (last2) $<3$ & $\begin{array}{l}\text { last } 2 \text { buffer never } \\
\text { overflows }\end{array}$ \\
\hline SAFE4 & $\begin{array}{l}\wedge \forall x \in \text { Range (signBuffer): IsWellFormedModbus }(x . \text { text }) \\
\wedge \forall x \in \text { Range (modchkBuffer): IsWellFormedModbus }(x . \text { text })\end{array}$ & $\begin{array}{l}\text { only well-formed } \\
\text { Modbus gets } \\
\text { forwarded }\end{array}$ \\
\hline SAFE5 & $\begin{array}{l}\wedge \forall x \in \text { Range }(\text { signBuffer }): \\
\quad \text { Cardinality }(\{y \in \text { Range }(\text { signBuffer }): x . i d=y . i d\})=1 \\
\wedge x \in \text { Range }(\text { modchkBuffer }): \\
\quad \text { Cardinality }(\{y \in \text { Range }(\text { modchkBuffer }): x . i d=y . i d\})= \\
1\end{array}$ & $\begin{array}{l}\text { each message that is } \\
\text { forwarded has a } \\
\text { unique message } \mathrm{id}^{*}\end{array}$ \\
\hline SAFE6 & $\begin{array}{l}\wedge \forall x \in \text { Range (signBuffer }): \\
\quad \exists y \in \text { Range (modchkBuffer }): x . i d=y . i d \\
\begin{array}{r}\wedge x \in \text { Range (modchkBuffer }): \\
\quad \exists y \in \text { Range }(\text { signBuffer }): x . i d=y . i d\end{array}\end{array}$ & $\begin{array}{l}\text { well-formed messages } \\
\text { get sent to both inner } \\
\text { components }\end{array}$ \\
\hline SAFE7 & $\neg(r \times B u f=\langle\rangle) \Rightarrow \operatorname{Head}(r \times B u f)=": "$ & $\begin{array}{l}\text { rxBuf is either empty } \\
\text { or starts with ":" }\end{array}$ \\
\hline LIVE1 & $\begin{array}{l}\text { IsWellformedModbus }(\mathrm{msg}) w \\
\qquad \exists x \in \text { range }(\text { signBuffer }): x . \text { text }=m s g\end{array}$ & $\begin{array}{l}\text { if the message is well- } \\
\text { formed then it gets } \\
\text { sent. This is weaker } \\
\text { than desired as it only } \\
\text { shows some message } \\
\text { exists, not necessarily } \\
\text { the same message. }\end{array}$ \\
\hline LIVE2 & $\diamond \square($ incomingMessages $=\langle\rangle)$ & $\begin{array}{l}\text { all messages are } \\
\text { processed }\end{array}$ \\
\hline LIVE3 & last $2=\langle " \backslash r ", " \backslash n "\rangle \rightsquigarrow$ last $2=\langle 0,0\rangle$ & $\begin{array}{l}\text { last } 2 \text { buffer gets reset } \\
\text { after each well-formed } \\
\text { message }\end{array}$ \\
\hline
\end{tabular}

Formalized properties are usually not simple and can take some effort to understand. The property SAFE2, given again in Equation (6), is a conjunction of two statements. Each line in the conjunction starts with $\wedge$, meaning "and". These two statements make use of a local symbol $x$. The first half of the first statement, the portion before the colon, can be read as "For all $x$, where $x$ is an element of the set signBuffer". SignBuffer is an ordered sequence of messages that are meant to be sent to the Crypto 
Table 11: TLA+ symbols used in the property definitions for trustnet_out

\begin{tabular}{|l|l|}
\hline $\begin{array}{l}\text { Symbol } \\
\text { rxBuf }\end{array}$ & $\begin{array}{l}\text { A buffer that holds the bytes that are received from the } \\
\text { network. This is a sequence. }\end{array}$ \\
\hline $\begin{array}{l}\text { Range() } \\
\text { signBuffer, modchkBuffer }\end{array}$ & $\begin{array}{l}\text { The buffers that hold messages "sent" to the respective inner } \\
\text { components. These are an abstraction as the full model } \\
\text { implements the message passing. }\end{array}$ \\
\hline last2 & $\begin{array}{l}\text { A sequence for keeping track of the last two characters in } \\
\text { rxBuf. For finding "Irln" }\end{array}$ \\
\hline IsWellformedModbus() & $\begin{array}{l}\text { Is true if the message length, starting, and ending characters } \\
\text { are all correct. }\end{array}$ \\
\hline msg & $\begin{array}{l}\text { The raw Modbus message being processed } \\
\text { The set of messages that will be received }\end{array}$ \\
\hline incomingMessages &
\end{tabular}

component. Note that SignBuffer does not contain raw Modbus. It holds message structures with fields for the raw Modbus, the message ID, and other piece of metadata that might need to be forwarded to a component. Following the colon, Len() returns the length of a sequence. Text is the field within the message structure that contains the raw Modbus, so the right of the colon is ensuring the length of the raw Modbus contained in x.text is less than or equal to the maximum size of a Modbus packet. The second line of the equation is the same property stated for the modchkBuffer that holds messages to be sent to the Modchk component. These properties are invariants; they are checked to be true in every state that is generated.

$\wedge \forall x \in$ Range(signBuffer) : Len (x. text) $\leq$ MAXMODBUSSIZE
$\wedge \forall x \in$ Range(modchkBuffer) $:$ Len(x. text) $\leq$ MAXMODBUSSIZE

Properties SAFE2 and SAFE4-6 are two properties ANDed to which the logical AND operator is applied as fulfillment of the general property requires checking the same

${ }^{3}$ In TLA+, as in mathematics, what a programmer calls a function is an array. The domain of the function is the set of numbers over which the function is defined. The range of the function is the set of values the function produces when a number from its domain is given as input. In programming terms, Range(T) returns the elements of an array in an unordered set. 
thing for two different buffers. SAFE5 shows that the ID sent is unique, but this property likely will need to be adjusted upon translation to real code as the implementation for a unique identifier will be limited to finite numbers and risks repeating. This property might be relaxed to an assumption that the ID implementation has a low repeat probability.

\section{Trustnet_out}

The thread responsible for collating messages from the inner components and sending them out to the trusted network is called trustnet_out and can be seen in Figure 22. This thread works from a FIFO queue that the inner components populate. Trustnet_out waits until a message $n$ is placed in its queue, then checks if a message with the same ID, message $m$, has already been received. Checking for the existence of message $m$ involves filtering a set ${ }^{4}$ for a message with the same ID. If the companion message has not been received, then $n$ is placed in the set of received messages and the loop repeats. If it has, and one or both messages have been marked as invalid, then both messages are discarded and the loop repeats. If both messages have been marked valid by the inner components, the raw Modbus is placed in the set of valid messages that have successfully traversed the device, finished_trustnet. Finished_trustenet abstractly represents the raw Modbus that has been printed to the serial port. The properties that need to be proven for this thread are seen in Table 12. The formalized property can be seen on the left and the informal description can be seen on the right.

${ }^{4}$ A set in TLA+ is the equivalent of a set in mathematics; it is unordered and potentially infinite. Using a set in the specification abstracts away the specific data structure chosen for the $\mathrm{C}$ code. The only stipulation is the method of the chosen data structure for finding an element must behave equivalently to filtering a set. 
Table 12: The desired properties of the Trustnet_out thread

\begin{tabular}{|c|c|c|}
\hline Name & Formalized Property in TLA+ & Informal Property \\
\hline SAFE1 & $\begin{array}{l}\wedge \forall x \in \text { Range (serialport) }: \\
\quad \exists y \in \text { Range }(\text { metaserialport }): y \cdot \text { text }=x \\
\begin{array}{r}\wedge y \in \text { Range (metaserialport }): \\
\quad \exists x \in \text { Range }(\text { serialport }): x=y . \text { text }\end{array}\end{array}$ & $\begin{array}{l}\text { items on the serial } \\
\text { port and the } \\
\text { metaserialport are } \\
\text { the same (weakly, } \\
\text { this checks for the } \\
\text { existance of a } \\
\text { message but its not } \\
\text { 1-1 mapping) }\end{array}$ \\
\hline SAFE2 & $\begin{array}{l}\forall x \in \text { Range (metaserialport }): \\
\wedge(\exists y \in \text { Range }(\text { MessagesFromInnerCells }): \\
\quad x . i d=y . \text { id } \wedge \text { y.isvalid } \wedge \text { y. source }=" \text { modchk" } \\
\wedge(\exists y \in \text { Range }(\text { MessagesFromInnerCells }): \\
\quad x . i d=y . \text { id } \wedge \text { y.isValid } \wedge \text { y. source }=\text { "verify" }\end{array}$ & $\begin{array}{l}\text { Only prints if both } \\
\text { inner components } \\
\text { say its valid }\end{array}$ \\
\hline SAFE3 & Len $($ txBuf $)<M A X M O D B U S S I Z E$ & $\begin{array}{l}\text { sending buffer never } \\
\text { overflows }\end{array}$ \\
\hline SAFE4 & $\begin{array}{l}\forall x \in \text { Range (serialport) }: \\
\quad \exists y \in \text { Range }(\text { MessagesFromInnerCells }): x=y . \text { text }\end{array}$ & $\begin{array}{l}\text { only valid Modbus } \\
\text { gets printed (this } \\
\text { module assumes } \\
\text { valid Modbus is } \\
\text { received from both } \\
\text { inner components }\end{array}$ \\
\hline LIVE1 & $\begin{array}{c}\forall x \in \text { Range(MessagesFromInnerCells): } \\
x \in \text { validMessages } \wedge x . i d=\text { msg.id } \\
\quad m x \notin \text { validMessages }\end{array}$ & $\begin{array}{l}\text { Each message that } \\
\text { gets its companion } \\
\text { message is sent or } \\
\text { discarded }\end{array}$ \\
\hline LIVE2 & $\begin{array}{c}\forall x \in \text { Range (MessagesFromInnerCells }): m s g=x \wedge \\
x . i s \text { alid } \wedge(\exists y \in \text { validMessages }: y . i d=x . i d \wedge \\
y . \text { isValid } \wedge \text { y. source } \neq x . \text { source }) \\
\quad \cdots z \in \text { Range }(\text { metaserialport }): \text { z.id }=x . i d\end{array}$ & $\begin{array}{l}\text { valid messages are } \\
\text { eventually sent }\end{array}$ \\
\hline LIVE3 & $\begin{array}{c}\forall x \in \text { Range(MessagesFromInnerCells }): \text { TRUE } \Rightarrow \\
\diamond(\exists y \in \text { validMessages }: y . i d=x . i d)\end{array}$ & $\begin{array}{l}\text { all messages are } \\
\text { eventually processed }\end{array}$ \\
\hline
\end{tabular}

An interesting note with LIVE1 is that it does not ensure that all messages that are received are sent out through the serial port. The Trustnet_in and Untrustnet_in modules can guarantee that messages they receive (provided the messages are valid) pass through to the inner components, but this component and Untrustnet_out rely on messages from two sources: Modchk and Crypto. These two sources are not defined in this TLA+ module, so no properties can be formulated based on their behavior. As such, the 
strongest liveness property that can be checked is that if message $x$ with id $i$ is received from one inner component, message $y$ with id $i$ is received from the other inner component, then those messages are acted upon (sent or discarded) then removed from the set of waiting messages. LIVE1 is restated formally in Equation 7. The variable validMessages is a misnomer as it does not contain just valid messages, but all messages that have been received from the inner components and are awaiting their corresponding message from the opposite component. It is a waiting room for unconsumed messages. A message could be stuck in this waiting room forever and the Trustnet_out specification would still be valid.

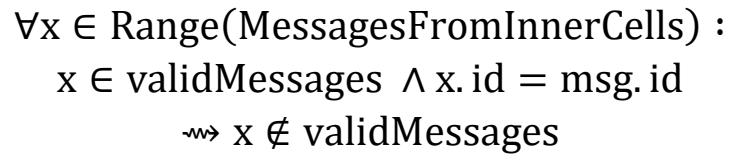

While TLA+ is very expressive, there are many expressions that are valid in TLA+ but which the TLC model checker refuses to evaluate. TLA+ uses mathematical notation, but in this dissertation the expressions are often describing actions rather than strictly equations. Actions relate to a state and a successor state, so certain variables at given states might not have been assigned at the time TLC tries to evaluate them. While working through the formalization of properties for this research, certain properties like LIVE1 were repeatedly reworked to account for these limitations. An earlier form of LIVE1 is given in Equation (8). This version is accepted by the semantics of TLA+, however in the initial state and any next-state computed from the initial state, validMessages has no value. The expression $x \in$ validMessages make sense mathematically, but TLC is unable to check it. To work around this, the possible values of $x$ were defined in the constant MessagesFromInnerCells seen in Equation (7) so the 
value assigned to $x$ does not depend on validMessages having been assigned a value. Describing all possible values of $x$ limits what can be checked, so care is taken to define values that allow the entire model to be checked for all properties. More discussion of the inner workings of TLC and this concern specifically can be found in chapter 14.2.6 of [64].

$\forall \mathrm{x} \in$ validMessages :

$\mathrm{x}$. id $=$ msg.id $\rightsquigarrow \mathrm{x} \notin$ validMessages

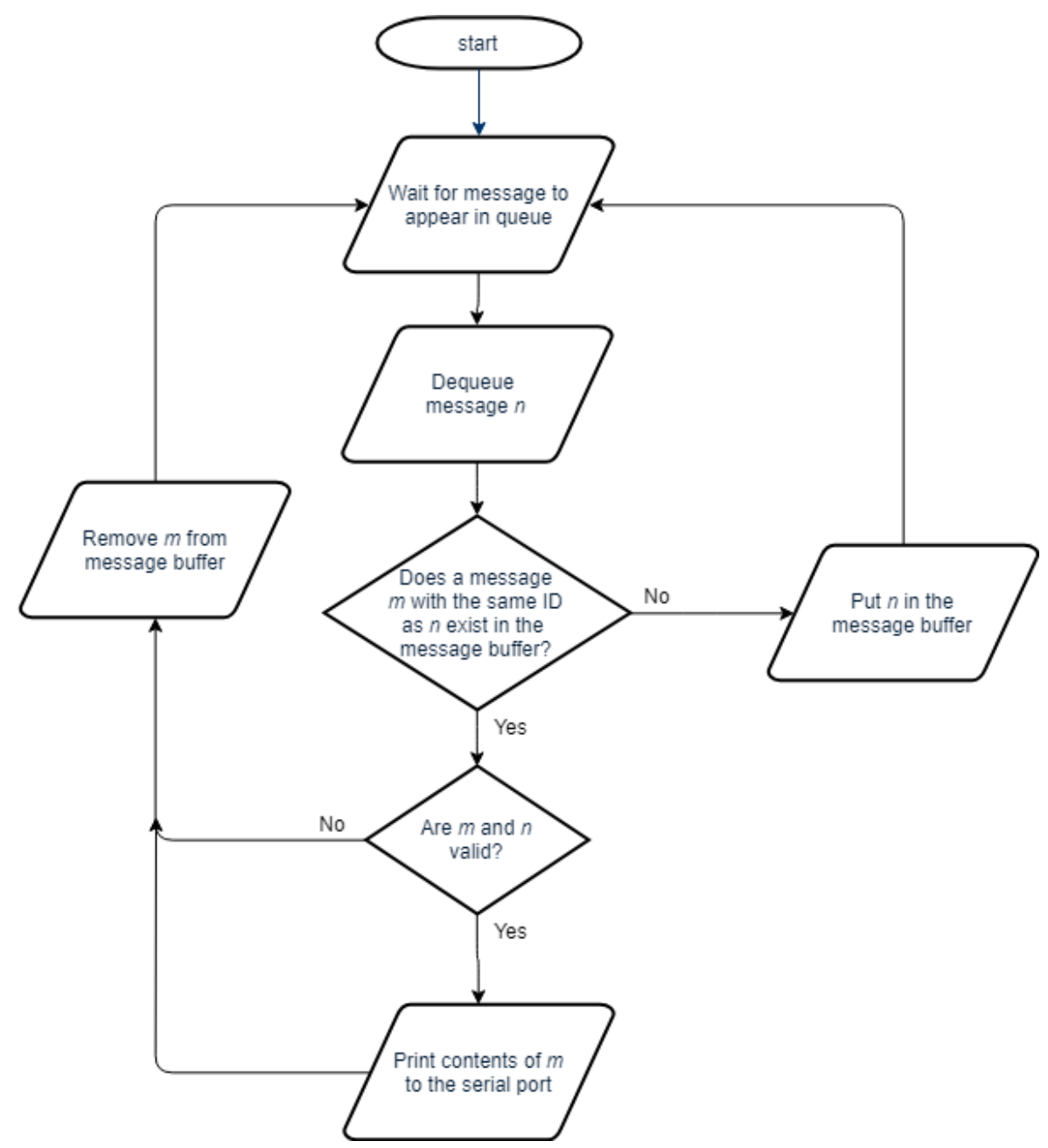

Figure 22: Flowchart for Trustnet_out thread 


\subsubsection{Modeling the Untrusted Network Component}

The untrusted networking component is similar to the trusted network component. As with the opposite network component design, the untrusted network component is separated into two processes: receiving messages from the untrusted network and sending messages on the untrusted network. The receiving process, called untrustnet_in, is designed to poll the serial port for input, so its logic is placed within a while loop that executes while there are messages to be processed. The algorithm for this thread is a basic loop, visualized in Figure 23, that will read a byte at a time from an incoming message and place it into a buffer. After processing is completed, the buffers are cleared and the thread starts again from the top. The more complicated parts come with the desired property that only well-formed Modbus messages reach the inner components. To achieve this, the logic checks each byte of input for "!", indicating the start of an encapsulated message. If a new encapsulated message is started before the previous one is finished, the previous message is discarded. Whenever a "!" is received, the contents of the buffer are flushed and the "!" is placed at the start of the empty buffer. Similarly, if the buffer is full despite not receiving a complete message, its contents are discarded, and the logic waits for a new "!". Secondly, the logic checks for the start of a well-formed Modbus message 64 characters later, starting after the HMAC. Next, the logic looks for the termination of a Modbus message. Encapsulated messages terminate with Modbus, and Modbus ASCII messages end with the two-byte sequence "Ir $\ln$ ". When this sequence is detected, this thread generates a structure containing the message, a generated message ID, and "trustnet_in" and places the object into the signing queue of Crypto. A similar structure is created with just the message and ID and placed in the queue of Modchk. 


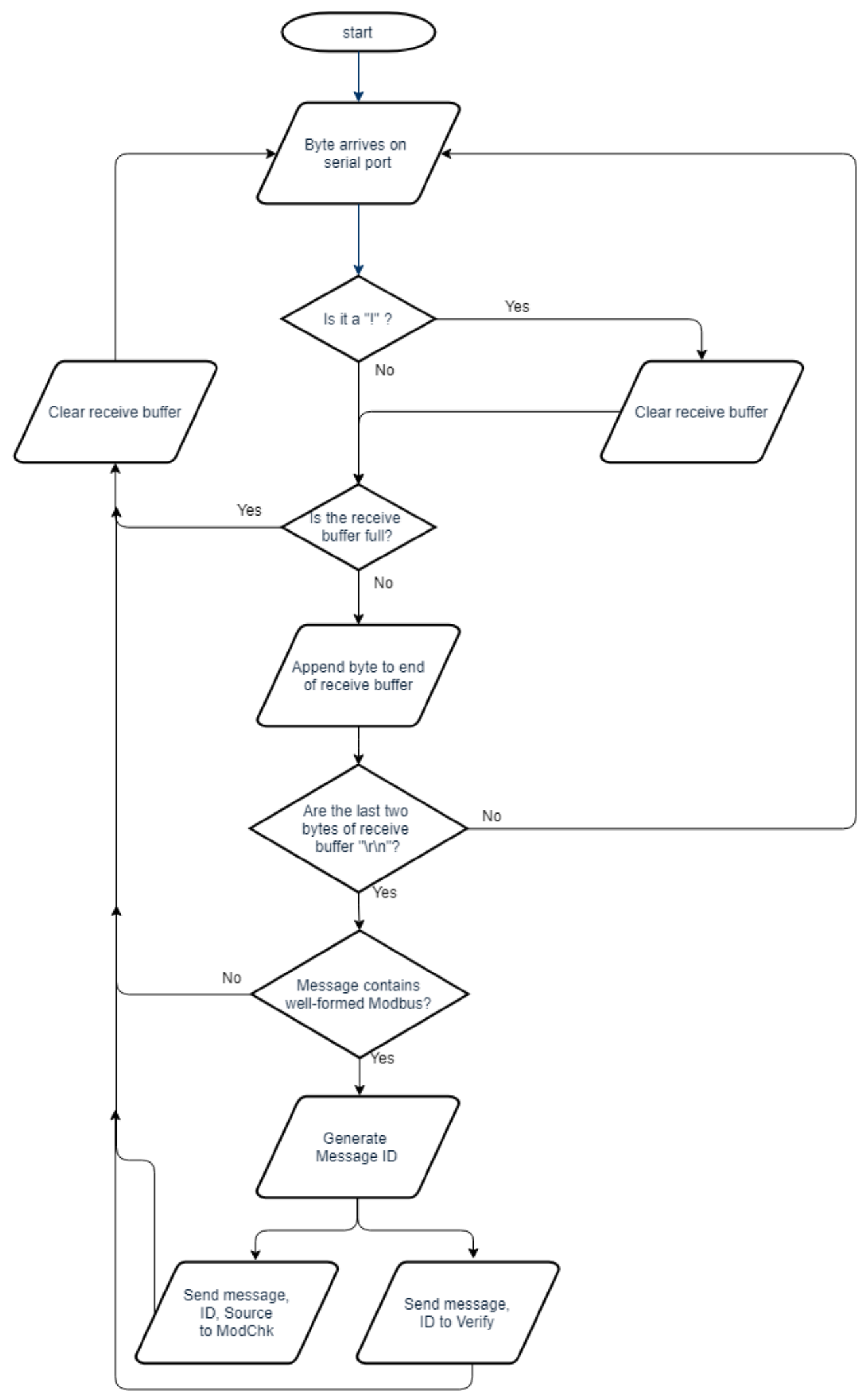

Figure 23: Flowchart for untrustnet_in thread 
The desired behavior of Unrustnet_in is that it accepts only and all well-formed encapsulated messages, forwards only well-formed Modbus, forwards the Modbus to both inner components in the same atomic step, forwards a single message exactly once, and doesn't overflow any buffers. The properties that need to be proven for this thread are seen in Table 13. The formalized property can be seen in the middle and the informal description can be seen on the right. Variables and operators in the properties seen in Table 13 are largely the same as those seen in Table 11, with the addition of IsWellformedEncap that checks for a starting "!", a terminating "Ir $\backslash n$ ", and that the message being checked is an appropriate length for encapsulated Modbus.

Table 13: Properties of the Untrustnet_in thread

\begin{tabular}{|c|c|c|}
\hline Name & Formalized Property in TLA+ & Informal Property \\
\hline SAFE1 & $\operatorname{Len}(r \times B u f) \leq M A X E N C A P S I Z E$ & $\begin{array}{l}\text { receive buffer never } \\
\text { overflows }\end{array}$ \\
\hline SAFE2 & $\begin{array}{l}\wedge \forall x \in \text { Range (verifyBuffer) }: \text { Len }(x . \text { text })<\text { MAXENCAPSIZE } \\
\wedge \forall x \in \text { Range(modchkBuffer }): \text { Len }(x . \text { text })<\text { MAXENCAPSIZE }\end{array}$ & $\begin{array}{l}\text { sending buffer never } \\
\text { overflows }\end{array}$ \\
\hline SAFE3 & Len $($ last 2$)<3$ & $\begin{array}{l}\text { last } 2 \text { buffer always } \\
\text { less than } 3\end{array}$ \\
\hline SAFE4 & $\begin{array}{l}\wedge \forall x \in \text { Range(verifyBuffer) }: \text { IsWellformedModbus }(x . \text { text }) \\
\wedge \forall x \in \text { Range(modchkBuffer) }: \text { IsWellformedModbus }(x . \text { text })\end{array}$ & $\begin{array}{l}\text { only well-formed } \\
\text { modbus gets } \\
\text { forwarded }\end{array}$ \\
\hline SAFE5 & $\begin{array}{l}\wedge \forall x \in \text { Range }(\text { verifyBuffer }): \\
\quad \text { Cardinality }(\{y \in \text { Range }(\text { verifyBuffer }): x . i d=y . i d\})=1 \\
\wedge x \in \text { Range }(\text { modchkBuffer }): \\
\quad \text { Cardinality }(\{y \in \text { Range }(\text { modchkBuffer }): x . i d=y . i d\})=1\end{array}$ & $\begin{array}{l}\text { each message that is } \\
\text { forwarded has a } \\
\text { unique message id }\end{array}$ \\
\hline SAFE6 & 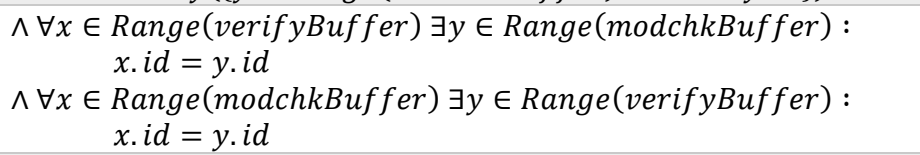 & $\begin{array}{l}\text { well-formed messages } \\
\text { get sent to both inner } \\
\text { components }\end{array}$ \\
\hline SAFE7 & $\neg(r \times B u f=\langle\rangle) \Rightarrow \operatorname{Head}(r \times B u f)=S T A R C H A R$ & $\begin{array}{l}\text { rxBuf is either empty } \\
\text { or starts with "!" }\end{array}$ \\
\hline LIVE1 & $\begin{array}{l}\text { IsWellformedEncap }(\mathrm{msg}) \\
\quad m \rightarrow \exists x \in \text { Range(verifyBuffer }): x . \text { text }=\text { msg }\end{array}$ & $\begin{array}{l}\text { if the message is well- } \\
\text { formed then it gets } \\
\text { sent }\end{array}$ \\
\hline LIVE2 & $\diamond \square($ incomingMessages $=\langle\rangle)$ & $\begin{array}{l}\text { all messages are } \\
\text { processed }\end{array}$ \\
\hline LIVE3 & last $2=\left\langle " \backslash r^{\prime \prime}, " \backslash n "\right\rangle{ }_{m \rightarrow}$ last $2=\langle 0,0\rangle$ & $\begin{array}{l}\text { last } 2 \text { buffer gets reset } \\
\text { after each well-formed } \\
\text { message }\end{array}$ \\
\hline
\end{tabular}


Liveness property LIVE2 has a structure that might be unfamiliar to those who do not work with temporal logic of actions. Liveness properties check that a given condition will eventually be met, and this one is checking that all messages that are received by Untrustnet_in are eventually processed in some way. LIVE2 starts with the temporal operator $\diamond$, indicating that the statement it precedes, $\square($ incomingMessage $=\langle\rangle)$, will eventually be true. Following the temporal operator is the invariant operator indicating the statement it precedes, (incomingMessage $=\langle\rangle$ ), is true for all states. Using these two operators in sequence indicates a property that eventually becomes true and stays true through program termination ${ }^{5}$. Informally, this property can be interpreted as "eventually, the sequence of incoming messages to be processed is emptied and stays empty".

\section{Untrustnet_out}

The thread responsible for collating messages from the inner components and sending them out to the untrusted network is called untrustnet_out and can be seen in Figure 24. This thread works from a FIFO queue that the inner components populate. Untrustnet_out waits until a message $n$ is placed in its queue, then checks if a message with the same ID, message $m$, has already been received. Checking for the existence of message $m$ involves filtering a set for a message with the same ID. If message $m$ has not been received, then $n$ is placed in the set of received messages and the loop repeats. If both messages $m$ and $n$ have been received and the message from the protocol checking

5 The property can oscillate between true and untrue as the state-trace unfolds, but eventually it becomes true through the end of the trace. 
component has been marked as invalid, both messages are discarded. If both messages $m$ and $n$ have been received and the message from the protocol checking component has been marked as valid, the HMAC is pulled from the opposite message. The full encapsulated message, a "!" concatenated with the HMAC and the raw Modbus, is placed in the set of valid messages that have successfully traversed the device, finished_untrustnet. Finished_untrustnet abstractly represents the encapsulated messages that has been printed to the serial port.

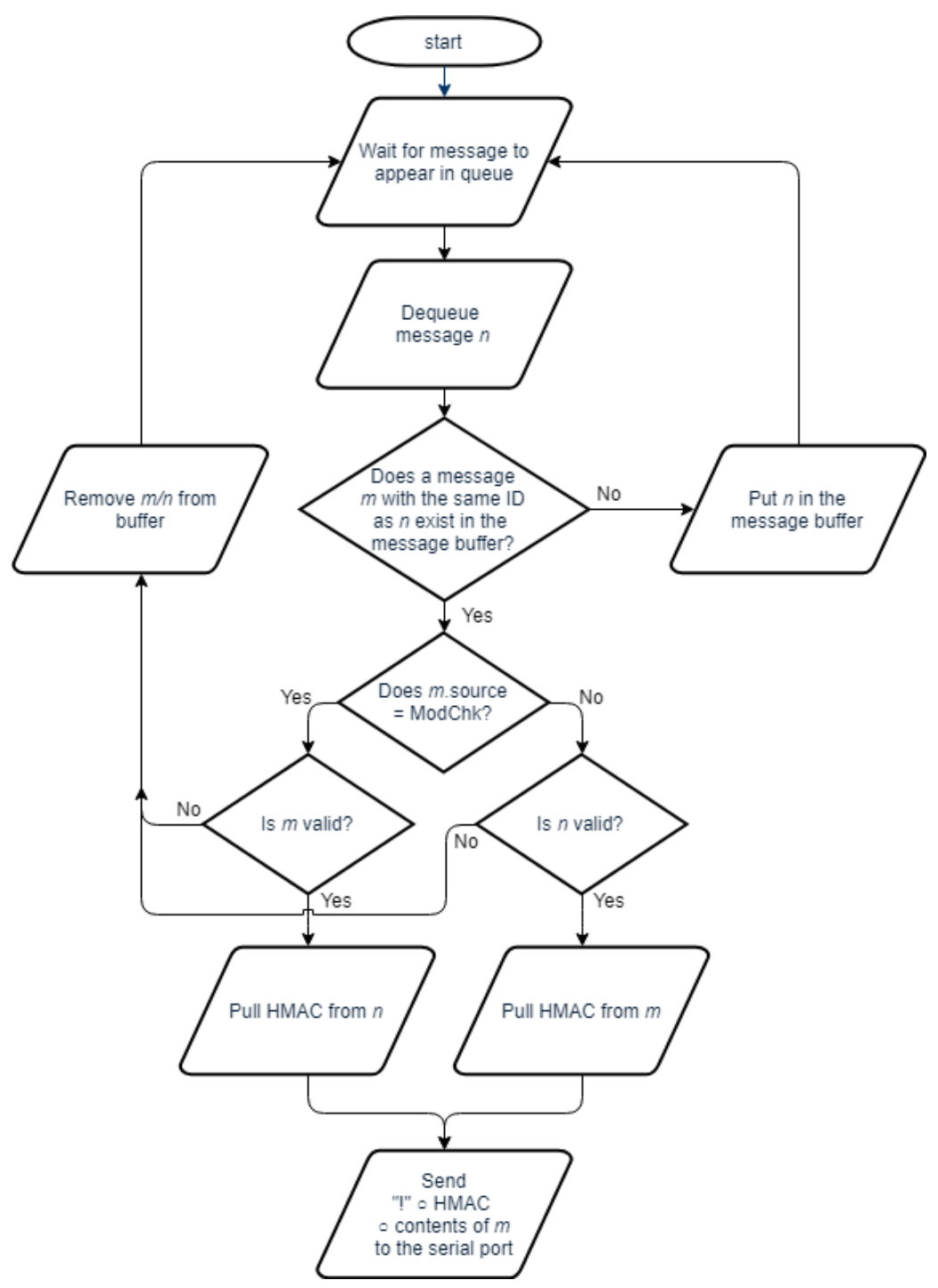

Figure 24: Flowchart for Untrustnet_out thread 
The properties that need to be proven for this thread are seen in Table 14. The formalized property can be seen on the left and the informal description can be seen on the right.

Table 14: The desired properties of the Unrustnet_out thread

\begin{tabular}{|c|c|c|}
\hline Name & Formalized Property in TLA+ & Informal Property \\
\hline SAFE1 & $\begin{array}{c}\wedge \forall x \in \text { range (serialport) }: \exists y \in \text { Range (metaserialport) } \\
:\langle!\rangle \circ \mathrm{HMAC} \circ \mathrm{y} \cdot \text { text }=\mathrm{x} \\
\begin{array}{r}\wedge \forall x \in \text { range (metaserialport) }: \exists y \in \text { Range (serialport) } \\
:\langle " !\rangle \circ \mathrm{HMAC} \circ \mathrm{y} \cdot \mathrm{text}=\mathrm{x}\end{array}\end{array}$ & $\begin{array}{l}\text { stuff on the serial port } \\
\text { and the metaserialport } \\
\text { are the same (weakly, } \\
\text { this checks for the } \\
\text { existance of a } \\
\text { message but its not } 1 \text { - } \\
1 \text { mapping) }\end{array}$ \\
\hline SAFE2 & $\begin{array}{l}\forall x \in \text { Range (metaserialport }): \\
\begin{aligned} \wedge(\exists y \in \text { Range }(\text { MessagesFromInnerCells }): x . i d \\
\quad=y . \text { id } \wedge \text { y. isValid } \wedge \text {. source } \\
=" \text { modchk" }\end{aligned} \\
\begin{array}{r}\wedge(\exists y \in \text { Range }(\text { MessagesFromInnerCells }): x . i d \\
=y . i d \wedge y . \text { isvalid } \wedge y . \text { source }=" \text { sign" }\end{array}\end{array}$ & $\begin{array}{l}\text { Only prints if both } \\
\text { inner components say } \\
\text { its valid }\end{array}$ \\
\hline SAFE3 & Len $(t x B u f)<$ MAXENCAPSIZE & $\begin{array}{l}\text { sending buffer never } \\
\text { overflows }\end{array}$ \\
\hline SAFE4 & $\begin{array}{l}\forall x \in \text { Range (serialport }): \\
\quad \exists y \in \text { Range }(\text { MessagesFromInnerCells }): \\
x=\langle " ! "\rangle \circ \text { HMAC } \circ \text { y.text }\end{array}$ & $\begin{array}{l}\text { only valid Modbus } \\
\text { gets printed (this } \\
\text { module assumes valid } \\
\text { Modbus is received } \\
\text { from both inner } \\
\text { components }\end{array}$ \\
\hline SAFE5 & $\neg($ txBuf $=\langle\rangle) \Rightarrow$ IsWellformedEncap $($ txBuf $)$ & $\begin{array}{l}\text { only well-formed } \\
\text { encap packets get } \\
\text { printed }\end{array}$ \\
\hline LIVE1 & $\begin{array}{c}\forall x \in \text { Range(MessagesFromInnerCells): } \\
x \in \text { validMessages } \wedge x \text {.id }=\text { msg.id } \\
m x \notin \text { validMessages }\end{array}$ & $\begin{array}{l}\text { Each message that } \\
\text { gets its companion } \\
\text { message is sent or } \\
\text { discarded }\end{array}$ \\
\hline LIVE2 & 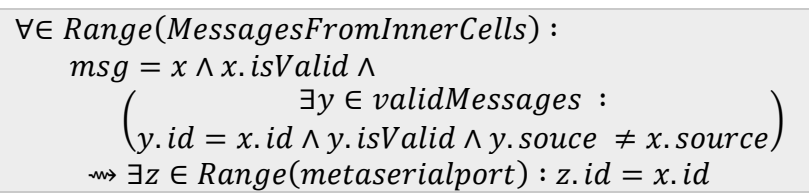 & $\begin{array}{l}\text { valid messages are } \\
\text { eventually sent }\end{array}$ \\
\hline LIVE3 & $\begin{array}{c}\forall x \in \text { Range (MessagesFromInnerCells }): \text { TRUE } \\
\quad \Rightarrow \diamond(\exists y \in \text { validMessages }: y . i d=x . i d)\end{array}$ & $\begin{array}{l}\text { all messages are } \\
\text { eventually processed }\end{array}$ \\
\hline
\end{tabular}

This component has an additional responsibility that Trustnet_out does not. Trustnet_out prints the raw Modbus message that is received from the inner component 
to the serial line and is only responsible for faithfully passing on the raw message as received without checks or modifications. In contrast, Untrustnet_out is responsible for encapsulating the raw Modbus received from the protocol checker with the HMAC received from Crypto. The extra invariant SAFE5 seen in Equation (9) is required to ensure this extra responsibility is correct. The symbol $t x B u f$ is the buffer that contains data to be printed to the serial port. The left-hand side of the equation is a negation of txBuf being empty. The right-hand side uses the custom operator Is WellformedEncap to determine if the data held in $t x B u f$ meets the specification of an encapsulated message. Informally, SAFE5 can be stated as "txBuf is either empty or contains a well-formed encapsulated message".

$$
\neg(\text { txBuf }=\langle\rangle) \Rightarrow \text { IsWellformedEncap(txBuf) }
$$

\subsubsection{Modeling the Protocol Checking Component}

Protocol checking in this system is intended to be somewhat flexible. Modbus was chosen for this dissertation for its familiarity and ease of use but is not the only protocol that sees use on industrial control systems. The desired property that only wellformed Modbus reaches the inner components of the system prevents the protocol checking from being completely modular as the networking components are coupled to the protocol, but the deep inspection is contained to the singular protocol checking component called Modchk. There are two pieces that act in composition that make up Modchk: 1) the portion that interacts with the other components to receive and pass along messages with the decision, and 2) the deep inspection that ensures every field of the raw Modbus conforms to the specification. There are likely many different customizations and extensions to the Modbus protocol that have been made to suit the mission demands 
at any given Modbus installation, so for this research the official stock Modbus specification given in [123] was chosen.

The first piece is a relatively simple model and can be visualized in the flowchart in Figure 25. This is a passive component, so it must be called upon by one of the network components to perform work. While the model and flowchart in Figure 25 express a message arriving in a queue, the seL4 component will receive a Remote Procedure Call (RPC). When a message arrives, it will contain the raw Modbus to be checked, a message ID, and a source identifier to indicate which networking component the message arrived from. The message is dequeued and passed to the predefined IsModbus operator. The output from IsModbus is attached to the message and forwarded to the opposite networking component.

The second piece is a more complex and formalizes the Modbus specification described in [123]. The TLA+ specification for Modbus does not produce a model that is checked, but rather it can be thought of as a formal definition for a raw Modbus ACSII message. The format for a Modbus ASCII message can be seen in Table 15. A separate operator has been defined for checking each field. The start and end fields are simply checked: the IsStart operator is true if the head of the message is ":" and the IsEnd

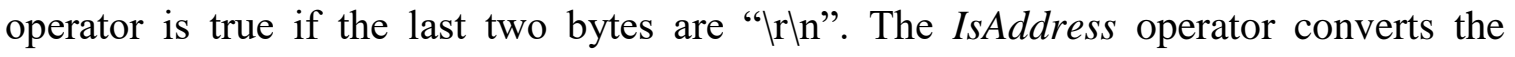
address from ACSII to decimal and checks that it is between 0 and 247. The IsFunctionCode operator converts the two bytes of ACSII function code data into decimal and checks that the result matches one of the 19 codes designated as public in [123], or a valid function code +127 to indicate an exception response. Certain function codes have sub-codes that add an additional two bytes to the function code field. These 
sub-codes are checked as well. Table 16 shows the function codes and sub-codes that are permissible in the IsFunctionCode operator. The IsData operator simply ensures that the data field is equal or fewer than 504 bytes as this data can vary in length and contents from transaction to transaction even with the same function code. Finally, IsLRC checks that the Longitudinal Redundancy Check (LRC) is accurate. This is accomplished by adding up the bytes that form the address, function code, and data fields, discarding all but the least significant byte of the result, then negating it. If the calculated LRC matches the LRC in the raw Modbus message, then IsLRC is true. Additionally, the entirety of the raw Modbus message is checked that each byte is a valid hexadecimal number. Each byte represents one hexadecimal digit in ACSII, so the byte 00100110 (38 in decimal and the "\&" character in ACSII) would not be a valid hex digit in ACII. Figure 26 shows the flowchart for the IsModbus operator. The order of checks is not defined in TLA+ so each sub-operator runs in one atomic step before the results of each is logically ANDed to produce the result.

Table 15: A Modbus ASCII message

\begin{tabular}{|c|c|c|c|c|c|c|}
\hline Start & Address & $\begin{array}{c}\text { Function } \\
\text { Code }\end{array}$ & Sub code & Data & LRC & End \\
\hline “:” & 2 bytes & 2 bytes & $\begin{array}{c}2 \text { bytes } \\
\text { (optional) }\end{array}$ & $\begin{array}{c}\text { Up to } \\
504 \text { bytes }\end{array}$ & 2 bytes & "Irln" \\
\hline
\end{tabular}

The properties that need to be proven for this thread are seen in Table 17. The formalized property can be seen on the left and the informal description can be seen on the right. 
Table 16: Modbus function codes. Adapted from [123]

\begin{tabular}{|c|c|c|c|c|c|c|}
\hline & & & & \multicolumn{2}{|c|}{ Function Codes } & \multirow[b]{2}{*}{ ex } \\
\hline & & & & Code & Sub-code & \\
\hline \multirow{13}{*}{ Data Access } & \multirow{4}{*}{$\begin{array}{l}\text { Bit } \\
\text { Access }\end{array}$} & $\begin{array}{l}\text { Physical } \\
\text { Discrete } \\
\text { Inputs } \\
\end{array}$ & Read Discrete Inputs & 02 & & 2 \\
\hline & & \multirow{3}{*}{$\begin{array}{l}\text { Internal Bits } \\
\text { or Physical } \\
\text { coils }\end{array}$} & Read Coils & 01 & & 1 \\
\hline & & & Write Single Coil & 05 & & 5 \\
\hline & & & Write Multiple Coils & 15 & & $\mathrm{~F}$ \\
\hline & \multirow{7}{*}{$\begin{array}{l}16 \mathrm{Bit} \\
\text { Access }\end{array}$} & \multirow{2}{*}{$\begin{array}{l}\text { Physical } \\
\text { Input } \\
\text { Registers }\end{array}$} & Read Input Register & 04 & & 4 \\
\hline & & & Read Holding Registers & 03 & & 3 \\
\hline & & \multirow{5}{*}{$\begin{array}{l}\text { Internal } \\
\text { Registers or } \\
\text { Physical } \\
\text { Output } \\
\text { Registers }\end{array}$} & Write Single Register & 06 & & 6 \\
\hline & & & Write Multiple Registers & 16 & & 0 \\
\hline & & & Read/Write Multiple Registers & 23 & & 7 \\
\hline & & & Mask Write Register & 22 & & 6 \\
\hline & & & Read FIFO queue & 24 & & 8 \\
\hline & \multirow{2}{*}{\multicolumn{2}{|c|}{ File Record Access }} & Read File record & 20 & & 4 \\
\hline & & & Write File record & 21 & & 5 \\
\hline \multirow{6}{*}{ Diagnostics } & & & Read Exception status & 07 & & 7 \\
\hline & & & Diagnostic & 08 & $00-18,20$ & 8 \\
\hline & & & Get Com event counter & 11 & & B \\
\hline & & & Get Com Event Log & 12 & & $\mathrm{C}$ \\
\hline & & & Report Server ID & 17 & & 1 \\
\hline & & & Read device Identification & 43 & 14 & B \\
\hline \multirow{2}{*}{ Other } & & & Encapsulated Interface Transport & 43 & 13,14 & B \\
\hline & & & CANopen General Reference & 43 & 13 & B \\
\hline
\end{tabular}




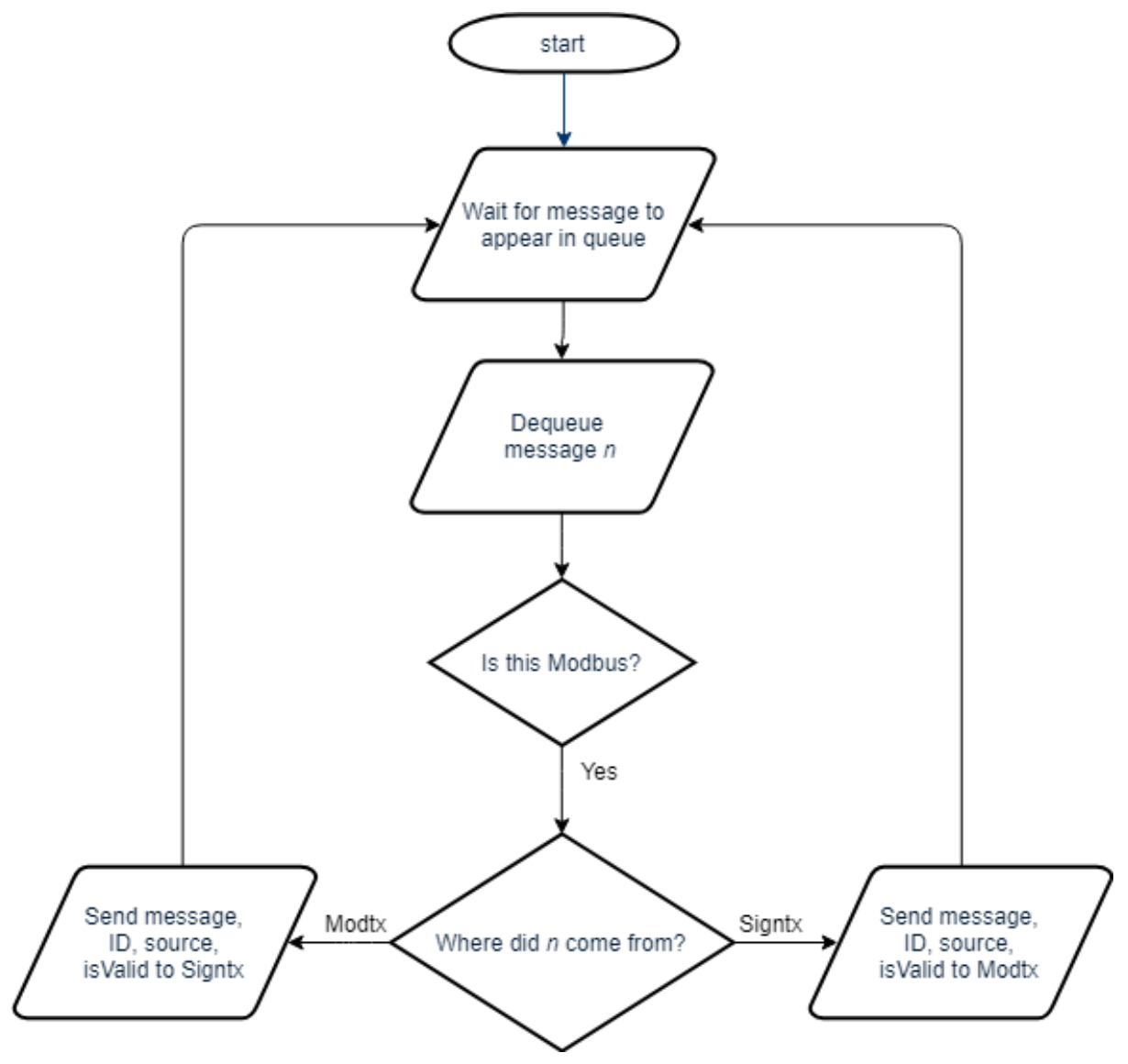

Figure 25: Flowchart for the Modchk component

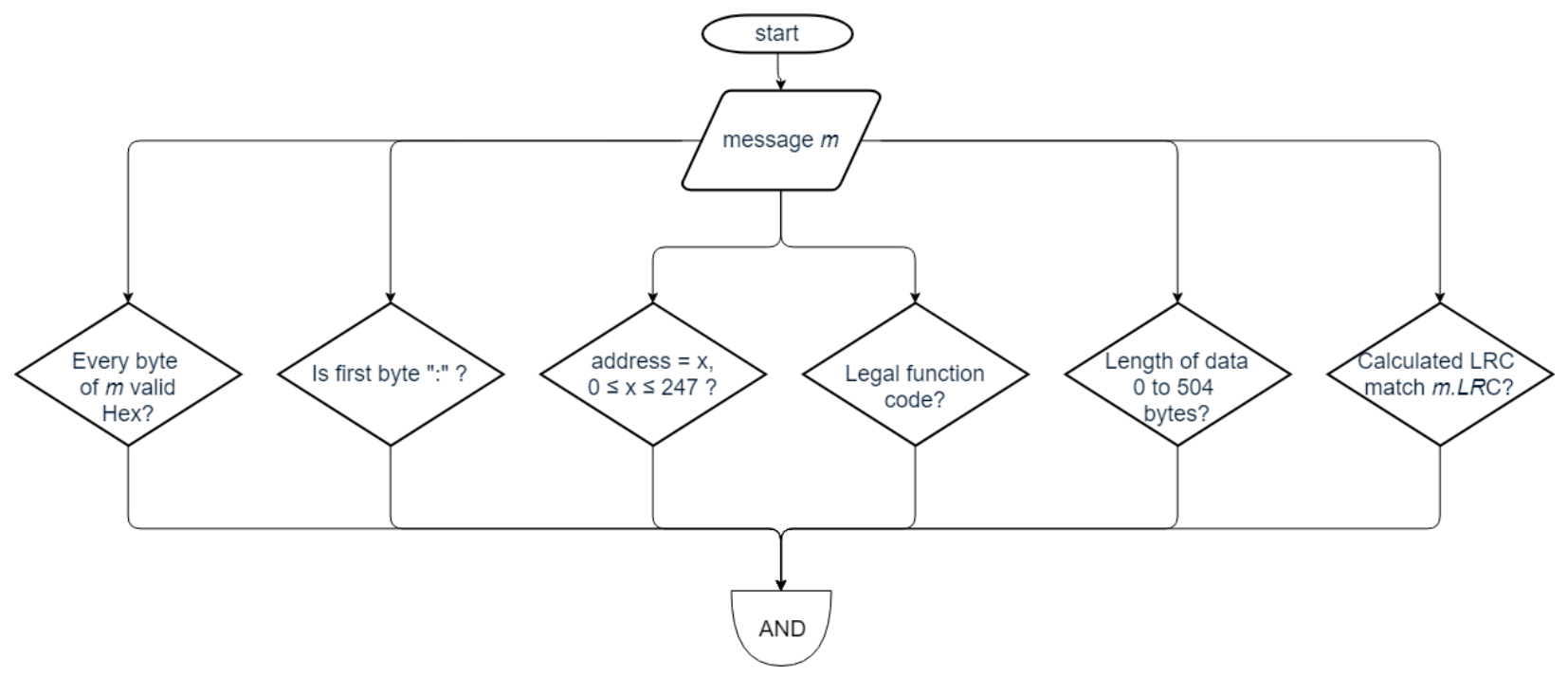

Figure 26: Flowchart for the IsModbus operator 
Table 17: The desired properties of the Modchk thread

\begin{tabular}{|c|c|c|}
\hline Name & Formalized Property in TLA+ & Informal Property \\
\hline SAFE1 & $\begin{array}{l}\forall x \in \text { ModbusMessages }: x . \text { source }=\text { trustnet_in } \\
\quad \Rightarrow(\exists y \in \text { Range }(\text { trustBuf }): y . i d=x . i d)=F A L S E\end{array}$ & $\begin{array}{l}\text { Messages from } \\
\text { untrustnet are } \\
\text { forwarded to } \\
\text { trustnet. This is two } \\
\text { parts, first an } \\
\text { invariant that says } \\
\text { no messages from } \\
\text { untrustnet will ever } \\
\text { make it into the set } \\
\text { of messages sent to } \\
\text { untrustnet }\end{array}$ \\
\hline LIVE1 & 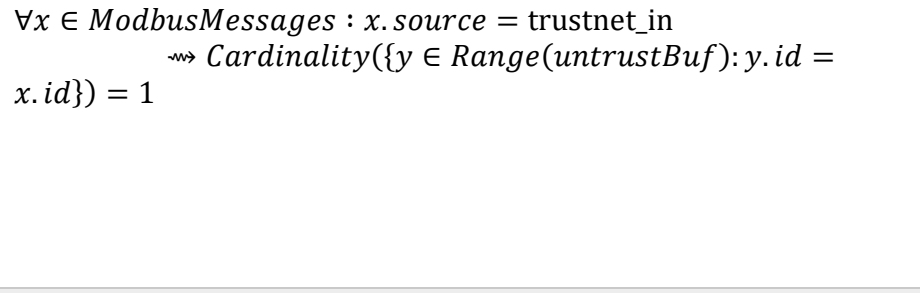 & $\begin{array}{l}\text { The second part is } \\
\text { that if a message is } \\
\text { from untrustnet, it } \\
\text { eventually will be } \\
\text { sent to trustnet } \\
\text { exactly once }\end{array}$ \\
\hline SAFE2 & $\begin{array}{l}\forall x \in \text { ModbusMessage }: x . \text { source }=\text { untrustnet_in } \\
\text { FALSE } \quad \Rightarrow(\exists y \in \text { Range }(\text { untrustBuf }): y . i d=x . i d)=\end{array}$ & $\begin{array}{l}\text { Same deal as above } \\
\text { but in reverse }\end{array}$ \\
\hline LIVE2 & $\begin{array}{l}\forall x \in \text { ModbusMessages }: \text { x. source }=\text { untrustnet_in } w \\
\quad \text { Cardinality }(\{y \in \text { Range }(\text { trustBuf }): y . i d=x . i d\})=1\end{array}$ & $\begin{array}{l}\text { Same deal as above } \\
\text { but in reverse }\end{array}$ \\
\hline SAFE3 & 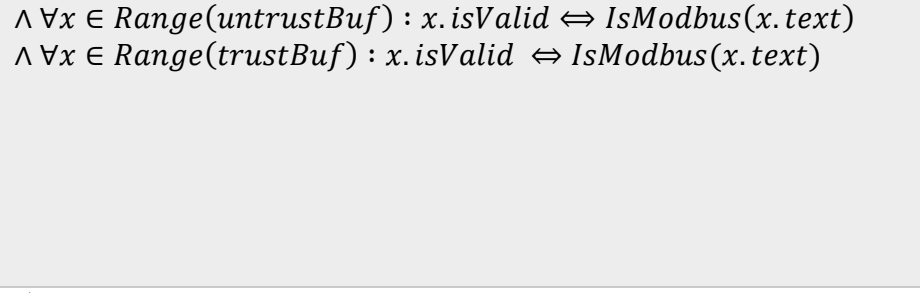 & $\begin{array}{l}\text { Malformed Modbus } \\
\text { is marked Invalid } \\
\text { when it leaves } \\
\text { Good Modbus is } \\
\text { marked valid when it } \\
\text { leaves }\end{array}$ \\
\hline LIVE3 & $\diamond \square($ incomingMessages $=\langle\rangle)$ & $\begin{array}{l}\text { if a message is } \\
\text { received it is } \\
\text { eventually processed }\end{array}$ \\
\hline
\end{tabular}

Invariant SAFE1 and temporal property LIVE1 work in tandem to check that a message received from one networking component is forwarded only to the opposite networking component exactly once. SAFE1, restated in Equation (10), states that for all messages in the set ModbusMessages (the set of messages that the model consumes for checking), if a message came from the trusted network, then it will never be found in the set of messages sent back to the untrusted network. This check is accomplished through 
use of the message IDs. The second line of SAFE1 states that there does not exist a message in the set of messages sent to the trusted networking component with an ID that matches a message that came from the trusted networking component. LIVE1 checks the second part of the desired property; that a received message is eventually forwarded to the opposite networking component. LIVE1, seen in Equation (11), shows a similar structure to Equation (10). It starts by referencing the set of all received Modbus messages and uses message IDs. Cardinality gives the number of elements in a set. The expression $\{y \in$ Range(untrustBuf): $y . i d=x . i d\}$ is the set of messages that have the same ID as $x \in$ ModbusMessages. The second line of LIVE1 thus states that the set of messages that have been sent to the untrusted network that have a matching ID as a give message that was received from the trusted network should contain exactly 1 element.

$$
\begin{aligned}
& \forall \mathrm{x} \in \text { ModbusMessages }: \mathrm{x} . \text { source }=\text { trustnet_in } \Rightarrow \\
& (\exists \mathrm{y} \in \text { Range }(\text { trustBuf }): \mathrm{y} . \mathrm{id}=\mathrm{x} . \mathrm{id})=\text { FALSE } \\
& \forall \mathrm{x} \in \text { ModbusMessages }: \mathrm{x} \text {. source }=\text { trustnet_in } m \\
& \text { Cardinality }(\{\mathrm{y} \in \text { Range(untrustBuf }): \mathrm{y} \cdot \mathrm{id}=\mathrm{x} . \mathrm{id}\})=1
\end{aligned}
$$

Formalizing the specification for Modbus ASCII and its encapsulation format lead to some insight into the design decisions found throughout the Modbus documents. Working with Modbus ASCII programmatically is a pain at first because there are three different formats for the data in use at a time: a byte of data might be represented in decimal form, in ASCII, or in ASCII hexadecimal characters. Modbus ASCII is communicated in the ASCII hexadecimal form with a single hex digit represented as a single ASCII character per byte; for example the single-byte hex value 0x3F would be represented as the two byte sequence " $3 F$ ". A single byte can hold two hex digits so Modbus ASCII creates double the necessary bandwidth by encoding 4 bits of data (a 
hexadecimal digit) into ASCII (a whole byte). In an ecosystem known for low power and minimal resources, doubling bandwidth consumption is not to be taken lightly. The earlier incarnation of Modbus, Modbus RTU, sends raw bytes as data with no special formatting. A single byte is used to communicate a single byte worth of data. When considering how to encapsulate a Modbus packet for transmission across the untrusted network, it became evident why Modbus ASCII encodes and transmits the data so inefficiently. When the switch was made to use a special character, “:”, to signify the start of a new Modbus ASCII message care had to be taken to ensure the selected special character could not organically appear within the Modbus ASCII message and cause the protocol to accidentally interpret a new message while in the middle of an existing message. For the encapsulated packet, "!" was chosen as a header character because the ASCII character "!" is not a valid Modbus ASCII hexadecimal character. The designers of Modbus ASCII decided to double the bandwidth and represent hex data as ASCII characters to make up for the decision to stop using a non-character-related transmission delay to signify a new message. This also comes into play when transmitting a cryptographic hash. A hash is just bytes of raw data that could be any value from 0 to 255. A Modbus message might not have the "!" character value even in its RTU variant, but the raw bytes of a hash certainly could. Doubling the length of the hash so it can be represented as ACSII hexadecimal eliminates this issue.

\subsubsection{Modeling the Cryptographic Component}

Application of cryptography is handled differently than the other pieces of the specification. Modeling the cryptographic algorithms has been done before in [86]-[88], and is beyond the scope of this research. However, modeling the behaviors of the threads 
that apply the cryptographic algorithms with the cryptography itself abstracted away can be done in a straight-forward fashion. The Crypto component has two threads: one for signing messages passing from a trusted network to an untrusted network, and one for verifying the signatures on messages passing through in the opposite direction.

The signing thread, called sign, is responsible for generating a keyed hash-based authentication code (HMAC) signature for each Modbus message that comes from the trusted network component. Its flowchart can be seen in Figure 27. The simplicity comes from abstracting the HMAC algorithm. Even though the message, secret key, and a unique nonce are passed to an HMAC function, the extent of the HMAC operator as defined in the model is returning a static 64-byte string. 64 bytes is the length of the output for SHA-256 so the proper length is important for determining if an encapsulated message is properly formed. Otherwise, the HMAC implementation is assumed to generate a unique 64-bit result for each message-secret-nonce combination.

The desired behavior for the sign thread is that every message is signed, no message is forwarded without an HMAC attached, the secret key never changes, and every message that is received is processed. The informal and formalized properties are shown in Table 18.

Properties LIVE2 and LIVE3 are subgoals for the larger property that every message that is received is passed through the Sign thread, no more and no fewer. There is no reason for Sign to be dropping messages so, unlike the network components that filter malformed messages, Sign will have a 1-to-1 mapping of input to output. The variable testmessages is a static set of messages that are used as input when calculating the state space and output holds the messages that pop out the other side of Sign. LIVE2 


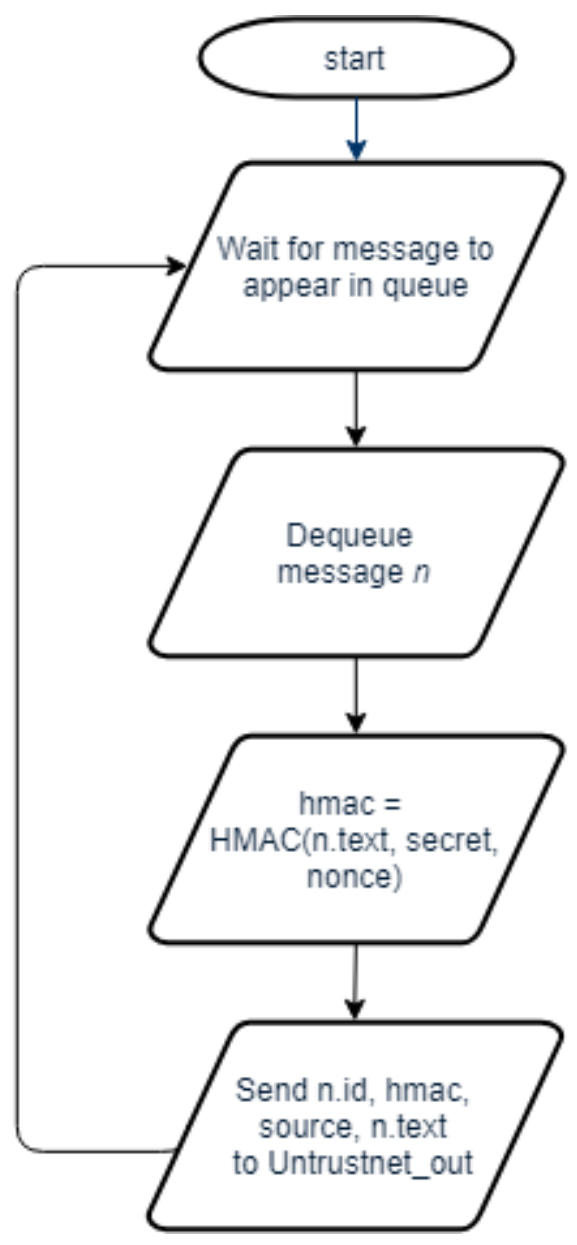

Figure 27: Flowchart for the Sign thread

Table 18: The desired properties of the Sign thread

\begin{tabular}{|c|c|c|}
\hline Name & Formalized Property in TLA+ & Informal Property \\
\hline SAFE1 & $\begin{array}{c}\forall x \in \text { Range(output) }: \exists y \in \text { Range(testmessages) }: y . \text { text } \\
=x . \text { text }\end{array}$ & $\begin{array}{l}\text { message sent is exactly } \\
\text { what was received }\end{array}$ \\
\hline SAFE2 & $\begin{array}{r}\forall x \in \text { Range (output) }: x \cdot h m a c \\
=H M A C(\text { msg.text, PASSWORD })\end{array}$ & output has good hash \\
\hline SAFE3 & 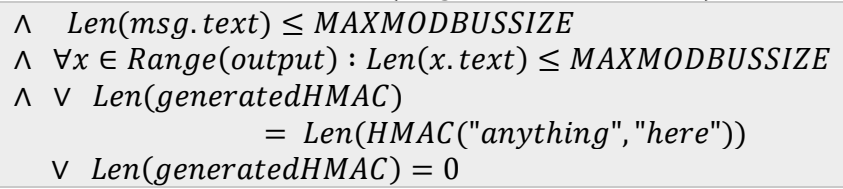 & buffers don't overflow \\
\hline SAFE4 & PASSWORD = "lolpassword" & password never changes \\
\hline LIVE1 & $\diamond \square$ Len $($ incomingMessages $)=0$ ) & $\begin{array}{l}\text { All messages are } \\
\text { eventually sent }\end{array}$ \\
\hline LIVE2 & $\diamond \square($ Len $($ output $)=$ Len $($ testmessages $))$ & $\begin{array}{l}\text { if we get a message then } \\
\text { something is eventually } \\
\text { sent }\end{array}$ \\
\hline LIVE3 & $\begin{array}{l}\forall x \in \text { Range }(\text { testmessages }): \\
\quad \diamond(\exists y \in \text { Range }(\text { output }): y . \text { text }=x . \text { text } \wedge y \cdot i d=x . i d)\end{array}$ & $\begin{array}{l}\text { if we get a message it is } \\
\text { eventually sent (part 2) }\end{array}$ \\
\hline
\end{tabular}


and LIVE3 thus combine to show the 1-to-1 mapping, and further the direct relation of messages, between testmessages and output. LIVE1 states the number of elements in output will become equal and stay equal to the number of elements in testmessages. LIVE3 states for all messages in testmessages, there will eventually exist a message with the same text and ID in output. LIVE2 is inadequate by itself as it could be true with all messages in testmessages being garbage. LIVE3 is inadequate by itself as it could be true with more messages than necessary, whether the extra messages are duplicates or garbage.

Verify

The verify thread makes similar abstractions with the implementation of the HMAC. The flowchart in Figure 28 shows a simple comparison operation as the heart of the thread. Within the received message is an HMAC that was (presumably) generated by the preprocessor at the other end of the line. The verify thread calculates the HMAC for the message itself using the message text, the secret key, and the nonce, the compares the received HMAC and the calculated HMAC. If these two values are equal, then the message is verified authentic and integrity is preserved. If these two values are different, then something is causing the message to be invalid. This is a critical decision and motivates the design of isolating this functionality within its own component.

The model as specified does not calculate an HMAC as the cryptographic algorithms are beyond the scope of this dissertation. With no calculated HMAC there is nothing to compare the received HMAC with, so the comparison is abstracted as well. This does not mean messages are marked valid or invalid randomly though, TLA+ and 
the TLC model checker have some cleverness that allows this abstraction without oversimplifying the model. The comparison variable is defined in Equation 12.

\section{CompareHMAC $\in$ BOOLEAN}

CompareHMAC is what is used in the critical decision of the verify thread. Its values can be anything in the BOOLEAN set \{true, false\}. TLA+ handles this assignment by branching, creating different behaviors for each possible value of CompareHMAC and checking every value independently. In the context of this specification, the verify thread branches on its critical decision and a new behavior to explore the states of the system that are reached for both a valid and invalid HMAC. Regardless of the result of the HMAC function that has been abstracted, the desired properties of the component and system at large must still hold. These properties are shown in Table 19 and are largely similar to the Sign thread.

Table 19: Desired properties of the Verify thread

\begin{tabular}{|c|c|c|}
\hline Name & Formalized Property in TLA+ & Informal Property \\
\hline SAFE1 & $\begin{array}{c}\forall x \in \text { Range(trustnetout) }: \exists y \in \text { Range(signedmessages) } \\
: y . \text { text }=x . \text { text }\end{array}$ & $\begin{array}{l}\text { message sent is exactly } \\
\text { what was received }\end{array}$ \\
\hline SAFE2 & $\begin{array}{cl}\wedge & I F \text { msg } \neq\langle\rangle \\
& \text { THEN Len }(\text { msg.text }) \leq \text { MAXMODBUSSIZE } \\
& \text { ELSE TRUE } \\
\wedge & \forall x \in \text { Range }(\text { trustnetout }): \text { Len }(x . \text { text }) \\
\leq & \leq \text { MAXMODBUSSIZE } \\
\wedge & \vee \text { Len }(\text { retreivedHMAC })=64 \\
& \vee \text { Len }(\text { generatedHMAC })=0\end{array}$ & buffers don't overflow \\
\hline SAFE3 & PASSWORD = "lolpassword" & $\begin{array}{l}\text { the password is never } \\
\text { changed }\end{array}$ \\
\hline LIVE1 & $\diamond \square$ Len $($ signedmessages $)=0$ ) & $\begin{array}{l}\text { All messages are } \\
\text { eventually sent }\end{array}$ \\
\hline LIVE2 & $\diamond \square($ Len $($ trustnetout $)=$ Len $($ signedmessages $))$ & $\begin{array}{l}\text { if we get a message then } \\
\text { something is eventually } \\
\text { sent }\end{array}$ \\
\hline LIVE3 & $\begin{array}{l}\forall x \in \text { Range (signedmessages }): \\
\quad \diamond(\exists y \in \text { Range }(\text { trustnetout }): y . \text { text }=x \cdot \text { text } \wedge y \cdot i d= \\
\text { x.id })\end{array}$ & $\begin{array}{l}\text { if we get a message it is } \\
\text { eventually sent (part 2) }\end{array}$ \\
\hline
\end{tabular}


One unfortunate part of leveraging TLA+'s branching and generating a new state for each of the two possible outcomes of the HMAC comparison is that there is no way or reason to ensure the HMAC is properly calculated and compared. The result of the "comparison", whether TRUE or FALSE, is picked at the time the comparison is made. If the properties were to try to make the comparison again, say to verify that the messages that are forwarded on to the next component are marked correctly, another random Boolean value will be chosen instead of the same value that was chosen for the initial comparison.

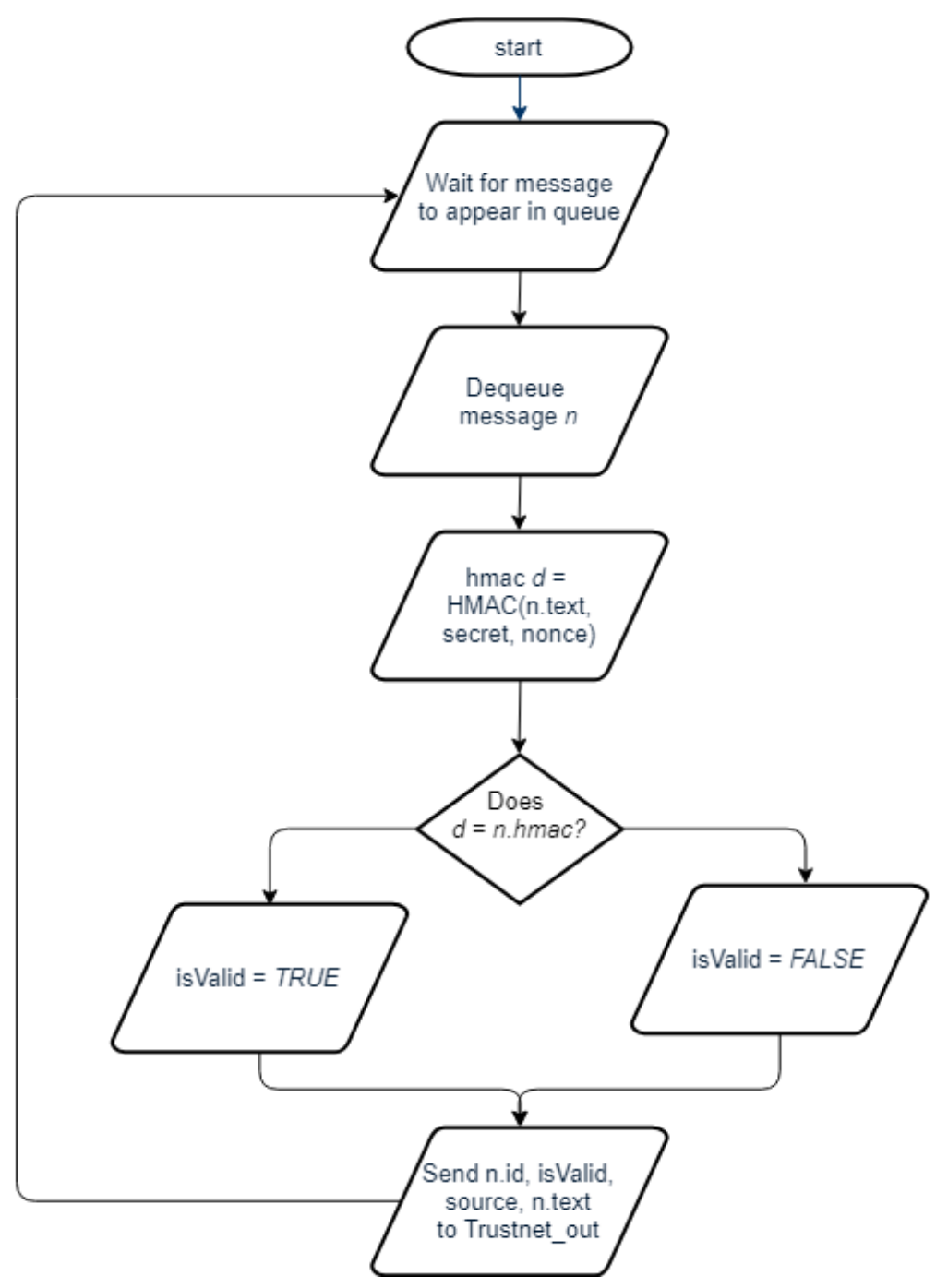

Figure 28: Flowchart for the Verify thread 


\subsubsection{Modeling the System}

Certain desirable properties of the system cannot be checked with the piecewise specifications described in this chapter. Properties such as LIVE1 of Trustnet_out (Table 12) that shows a message will pass through Trustnet_out (either printed or discarded) if it is received from both inner components is too weak to be useful by itself. A stronger and more useful property is that every message that is received by Trustnet_out is eventually printed or dropped. The specification of Trustnet_out is not capable of proving this stronger property because it has no way of controlling the inputs from the inner components, no way of ensuring it receives a decision from both of them. This is where combining each of the piecewise specifications into a single, larger specification is useful. A composite specification can show that Trustnet_in will eventually receive a message from both inner cells, therefore demonstrating the stronger property. There are a few such properties that require a system-wide view to be proven. This section will describe how the components are combined, considerations for state-space of a larger specification, and writing new desired properties.

TLA+ allows defining processes that can run in parallel. In practice, this means that the atomic steps within each process have no defined execution order and TLA+ may choose any next-step to execute at any time. Each module defined for each component operates within its own process. The trusted networking component comprises a processes for reading the serial port and a process for writing to the serial port, the untrusted networking component comprises a process for reading the serial port and writing to the serial port, the cryptographic component comprises a process for signing messages and verifying signatures, and the protocol checking component comprises a 
single process for validating messages. Each process communicates with each other process via first-in-first-out (FIFO) queues. Inter process communication from process $a$ to process $b$ is abstractly modeled with $a$ placing a message in the FIFO queue of $b$. When $b$ finishes its processing steps with its current input, it either dequeues a message from its assigned queue or blocks until its queue is non-empty. For this model a statetrace is complete when all processes are blocked.

Detailing each process within the security preprocessor specification would be redundant so the focus here will be on the desired macroscopic properties as laid out in Chapter 3. They are restated in Table 20.

Table 20: Desired informal properties of the security preprocessor

\begin{tabular}{|c|c|}
\hline Property & Description \\
\hline 1 & $\begin{array}{l}\text { Isolation between components such that a compromised component cannot } \\
\text { affect any others }\end{array}$ \\
\hline 2 & $\begin{array}{l}\text { Only proper messages can be allowed to pass through - no malformed } \\
\text { messages }\end{array}$ \\
\hline 3 & Only properly formed messages can originate from the device \\
\hline 4 & Every message is properly authenticated \\
\hline 5 & Every message is protected from tampering \\
\hline 6 & $\begin{array}{l}\text { All legitimate messages do eventually pass through (except in the case of } \\
\text { denial of service) }\end{array}$ \\
\hline
\end{tabular}

Property 1 is granted automatically through the use of the seL4 microkernel. Properties 2-5 are invariants and property 6 is temporal. Properties 2 and 3 are similar but different in that property 2 deals with messages that the preprocessor receives from the network. Property 3 concerns with messages that might be created by the preprocessor if it were compromised in some way. Both 2 and 3 are handled by the specification of the networking components. Their design requirements state that they are only able to print 
well-formed (not necessarily valid) messages. Properties 4 and 5 require a general specification; the cryptographic component can ensure it does its job for every message it encounters, but a general specification is required to show that every well-formed message from the network ever reaches the cryptographic component. Finally, Property 6 gains the most from a general specification. Each component has been shown to eventually process all messages they encounter, but the nature of parallel critical decisions in the cryptographic and protocol checking components requires both be modeled in the same specification to ensure the networking components receive the decision from both of them. The general specification takes a set message end-to-end through the preprocessor model to check this property. The formalized properties and their subgoals can be seen in Table 21 .

Table 21: The desired formal properties of the security preprocessor

\begin{tabular}{|c|c|c|}
\hline Name & Formalized Property in TLA+ & Informal Property \\
\hline SAFE1 & $\begin{aligned} \forall m \in \text { Range } & \text { (chan [messagecheck] } \\
& : \text { Len }(\text { m.text }) \\
& \leq \text { MAXMODBUSSIZE }\end{aligned}$ & modbus check module: \\
\hline SAFE2 & $\forall m \in$ validMessages $:$ m.isValid & $\begin{array}{l}\text { message parts waiting } \\
\text { for their counterpart } \\
\text { are valid }\end{array}$ \\
\hline SAFE3 & $\begin{array}{l}\forall m \in \text { Range (chan[finished_untrustnet] }) \\
\quad: \operatorname{Get} H M A C(m)=H M A C(m, m)\end{array}$ & $\begin{array}{l}\text { HMACs are properly } \\
\text { applied }\end{array}$ \\
\hline SAFE4 & $\begin{array}{r}\forall m \in \text { Range (chan[finished_untrustnet] }) \\
\quad: \text { IsModbus }(\text { GetMessage }(m))\end{array}$ & $\begin{array}{l}\text { only properly signed } \\
\text { messages are sent to } \\
\text { untrustnet }\end{array}$ \\
\hline SAFE5 & $\forall m \in$ validMessages $:$ m. isValid & $\begin{array}{l}\text { message parts waiting } \\
\text { for their counterpart } \\
\text { are valid }\end{array}$ \\
\hline SAFE6 & $\begin{array}{c}\forall m \in \text { Range(chan[finsihed_trustnet]) } \\
: \operatorname{IsModbus(m)}\end{array}$ & $\begin{array}{l}\text { only properly formed } \\
\text { modbus is sent to } \\
\text { trustnet }\end{array}$ \\
\hline LIVE1 & $<>($ Len $($ incomingMessages_ $)=0)$ & $\begin{array}{l}\text { All messages } \\
\text { eventually processed } \\
\text { from trustnet }\end{array}$ \\
\hline LIVE2 & $<>($ Len $($ incomingMessages $)=0)$ & All messages \\
\hline
\end{tabular}




\begin{tabular}{|l|l|l|}
\hline LIVE3 & $<>(\operatorname{Len}(\operatorname{chan}[$ finsihed_untrustnet] $)>0)$ & $\begin{array}{l}\text { eventually processed } \\
\text { from untrustnet }\end{array}$ \\
\hline LIVE4 & $<>\operatorname{Len}($ chan[finsihed_trustnet] $)>0$ & $\begin{array}{l}\text { Messages pass through } \\
\text { device going one way }\end{array}$ \\
\hline & $\begin{array}{l}\text { Messages pass through } \\
\text { the device going the } \\
\text { other way }\end{array}$ \\
\hline
\end{tabular}

\subsubsection{Additional Operators and Functions in TLA+}

Modeling the desired security properties of the security preprocessor allowed the cryptographic algorithms to be reduced to an abstraction, but the protocol checking and networking components required a deeper level of implementation. This section discusses some of the helper operators and functions that were developed in TLA+ to assist in the model checking. The definitions presented here are novel contributions, though they are not directly relevant to the research in the previous sections of this chapter.

\section{American Standard Code for Information Interchange (ACSII)}

American Standard Code for Information Interchange (ACSII), is an encoding standard for representing characters from the English language and electronic transmission codes as numbers to facilitate digital communication. The portion of the ACSII standard used here is limited to 7-bits of data capable of representing 128 different characters seen in Appendix x. This research opted to concretely model the inputs and outputs of the serial ports. The selected protocol is Modbus ACSII, so a few helper functions were created to help with the specifics of ACSII. Firstly, a definition for a sequence of usable ACSII characters. The contiguous characters from $32_{10}$ to $126_{10}$ that might be seen in a Modbus ASCII network are seen in TLA+ Snippet 1. This definition requires an ordered sequence rather than a set because the position of each element matters for conversion back and forth from ACSII to decimal representation. 


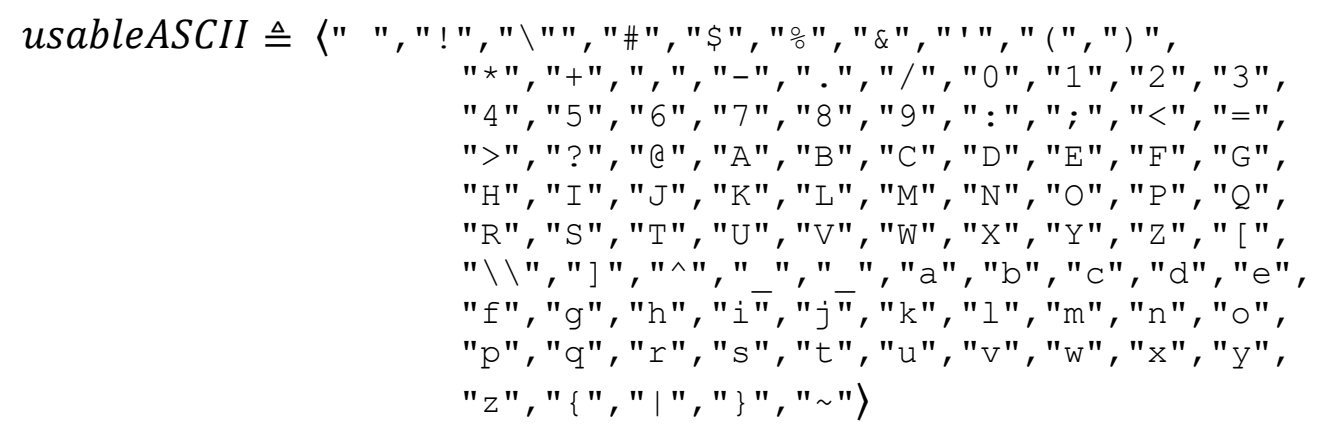

TLA+ Snippet 1: The definition of usableACSII

There are a few special characters used in Modbus ACSII communications that are not part of the contiguous block of usable ACSII. Their order is not as relevant, so their definition is a set shown in TLA+ Snippet 2. Symbols included in the set of special characters are $\downarrow t$ for tab, $\backslash r$ for carriage return, $\vee n$ for line feed, and $\downarrow$ for form feed.

$$
\text { specialChars } \triangleq\{" \backslash t ", ~ " \backslash r ", ~ " \backslash n ", ~ " \backslash f "\}
$$

TLA+ Snippet 2: The definition of specialChars

With the sequence usableACSII and the set specialChars, the set of relevant ACSII can be easily defined as follows:

$$
\text { setOfASCII } \triangleq \text { Range(usableACSII) U specialChars }
$$

TLA+ Snippet 3: The definition of setOfACSII

Conversion from decimal representation to ASCII representation is necessary for easier understanding of messages while writing and debugging the specifications. The specification for the preprocessor is designed to operate on the decimal representation that would be received from the serial port. Converting the messages to ACSII makes them human-readable. Converting from an ASCII character to a decimal number is done using CharToNum in TLA+ Snippet 4. TLA+ keyword CHOOSE selects a single element from the set constructed from the right-hand side of the colon; in this case the set should only contain a single number that maps to the input character in usableACSII. 
CharToNum(char) $\triangleq I F$ char $\in$ Range (usableACSII)

$$
\begin{aligned}
& \text { THEN } 31+\text { CHOOSE } i \in 1 . .95: \text { usableACSII }[i]=\text { char } \\
& \text { ELSE CASE char }=" \backslash t " \rightarrow 9 \\
& \square \text { char }=" \backslash r " \rightarrow 13 \\
& \square \text { char }=" \backslash n " \rightarrow 10 \\
& \square \text { char }=" \backslash f^{\prime \prime} \rightarrow 12 \\
& \square \text { OTHER } \rightarrow 0
\end{aligned}
$$

TLA+ Snippet 4: The CharToNum operator

Conversion from ACSII to decimal is similarly achieved with NumToChar see in TLA+ Snippet 5.

NumToChar $($ num $) \triangleq I F$ num $\in 32 . .126$

$$
\begin{aligned}
& \text { THEN usableACSII [num - 31] } \\
& \text { ELSE CASE num }=9 \rightarrow " \backslash t " \\
& \square \text { num }=13 \rightarrow " \backslash r " \\
& \text { 口num }=10 \rightarrow " \backslash n " \\
& \text { 口num }=12 \rightarrow \text { "\f" } \\
& \text { 口OTHER } \rightarrow \text { "" }
\end{aligned}
$$

TLA+ Snippet 5: The NumtoChar operator

NumtoChar and CharToNum were never really used by themselves. Rather, they were used to convert a sequence of numbers or characters (a message) from one format to the other. NumTupleToStrTuple and StrTupleToNumTuple in TLA+ Snippet 6 are functions designed to map ASCII to decimal for sequences.

$$
\begin{aligned}
& \text { NumTupleToStrTuple(numTuple) } \triangleq[x \in \text { DOMAIN numTuple } \\
& \mapsto \text { NumToChar (numTuple }[x])] \\
& \text { StrTupleToNumTuple }(\text { str }) \triangleq[x \in \text { DOMAIN str } \mapsto \text { CharToNum }(\operatorname{str}[x])]
\end{aligned}
$$

TLA+ Snippet 6: The NumTupleToStrTuple and StrTupleToNumTuble functions

One of the properties of a valid Modbus ASCII message is that every character in the message is a valid ASCII character. Checking that every character in a message is a valid Modbus ASCII character is accomplished ensuring each character is an element of setOfASCII shown in TLA+ Snippet 7. 


$$
\text { IsUsableASCII }(\text { str }) \triangleq s t r=S e l e c t S e q(s t r, L A M B D A x: x \in \text { SetOfASCII }
$$

TLA+ Snippet 7: The IsUsableACSII operator

After checking ASCII and decimal representations, checking sequences, and checking sets, the final thing to check is sanity. In theory, the operator for converting from ASCII to decimal should be inverses of one another. Checking sanity means ensuring the conversion of the sequence of ASCII chars to decimal and back again should yield the same sequence. The sanity check is shown in TLA+ Snippet 8 .

$$
\begin{aligned}
\text { SanityCheck } & \triangleq \text { usableASCII } \\
& =\text { NumTupleToStrTuple(StrTupleToNumTuple(usableACSII)) }
\end{aligned}
$$

TLA+ Snippet 8: The SanityCheck operator

\section{Hexadecimal}

Modbus ACSII is printed to the serial line in hexadecimal. Certain operations involving Modbus ASCII require manipulating hexadecimal values so a hex module was adapted and expanded from Andrew Helwer's Hex.tla in [141]. A TLA+ formula for converting from the ASCII representation of a string of hexadecimal digits to a sequence of base-10 digits is needed for calculating the longitudinal redundancy check (LRC). ASCIIHexToDecimal (TLA+ Snippet 9) takes as input a sequence of ASCII hex digits and calculates a sequence of decimals. In practice, two bytes of Modbus ASCII represents one byte of data. This formula thus converts the first two characters into a single decimal before recursing on the rest of the input. StringToHex is a simple formula that maps the character "1" to the number 1 , "2" to 2 , and so on. The symbol $\circ$ means append. 


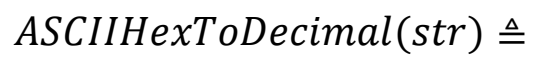

$$
\begin{aligned}
& \text { IF str }=\langle\rangle \\
& \text { THEN }\langle\rangle \\
& \begin{aligned}
\text { ELSE }\langle\text { StringToHex }(\operatorname{str}[1]) * 16 \\
\quad+\text { StringToHex }(\operatorname{str}[2]\rangle \\
\quad \\
\quad \text { OASCIIHexToDecimal(Tail }(\text { Tail }(\text { str })))
\end{aligned}
\end{aligned}
$$

TLA+ Snippet 9: The ACSIIHexToDecimal operator

\section{Longitudinal Redundancy Check}

Longitudinal Redundancy Check (LRC) is an algorithm for detecting transmission errors often used in serial communication. Its simplicity allows for quickly checking if part of a message has been lost or interfered with but does not try to fix any errors and does not protect against intentional tampering. LRC operates on bits, so the ASCII, hexadecimal, and decimal formats used throughout this research need to be converted before applying LRC. Hex sequences can be converted to decimal with ASCIIHexToDecimal. Likewise, ASCII sequences can be converted to decimal with StrTupleToNumTuple and CharToNum. To obtain bits (big-endian), any format in use must first be converted to decimal, then the decimal format can be converted to bits using DecimalToBinarySeq (TLA+ Snippet 10). The \langle\rangle indicate sequences.

$$
\begin{aligned}
\text { DecimalToBinarySeq }(\text { num }) \triangleq\langle\text { num } \div 128 \% 2\rangle & \circ\langle\text { num } \div 64 \% 2\rangle \\
& \circ\langle\text { num } \div 32 \% 2\rangle \\
& \circ\langle\text { num } \div 16 \% 2\rangle \\
& \circ\langle\text { num } \div 8 \% 2\rangle \\
& \circ\langle\text { num } \div 4 \% 2\rangle \\
& \circ\langle\text { num } \div 2 \% 2\rangle \\
& \circ\langle\text { num } \% 2\rangle
\end{aligned}
$$

TLA+ Snippet 10: The DecimalToBinarySeq operator

The inverse of DecimalToBinarySeq is the formula BinarySeqToDecimal (TLA+ Snippet 11). This formula takes as input a sequence of $\mathrm{N}$ bits and multiples the least 
significant bit by $2^{0}$. It then adds the result to the recursion of the formula calculated with the first $N-1$ bits and $2^{1}$, the first $N-2$ bits and $2^{2}$, and so on.

$$
\begin{aligned}
& B 2 D(\text { num }, \text { seq }) \triangleq I F \text { seq }=\langle\rangle \\
& \text { THEN } 0 \\
& \quad \begin{array}{l}
\text { ELSE }(\text { seq }[\text { Len }(\text { seq })] * \text { num })+B 2 D(2 \\
\\
* \text { num, SubSeq }(\text { seq }, 1, \text { Len }(\text { seq })-1))
\end{array} \\
& \text { BinarySeqToDecimal }(\text { seq }) \triangleq B 2 D(1, \text { seq })
\end{aligned}
$$

TLA+ Snippet 11: The BinarySeqToDecimal operator and its helper function B2D

The LRC algorithm is relatively simple bit arithmetic and manipulation. LRC takes as input a sequence of bytes representing a message. The bytes are added together and all but the least significant byte of the sum is discarded. The least significant byte is negated using Two's compliment to produce the LRC value. The sum of all the bytes that comprise the address, function code, and data fields of a Modbus message AND FF plus the LRC value should equal 0 . Formula addSeq is used to add each element of a decimal sequence in TLA+ Snippet 12.

$$
\begin{aligned}
& \operatorname{addSeq}(\text { seq }) \triangleq I F \text { seq }=\langle\rangle \\
& \text { THEN } 0 \\
& \text { ELSE Head }(\text { seq })+\operatorname{addSeq}(\operatorname{Tail}(\text { seq }))
\end{aligned}
$$

TLA+ Snippet 12: The addSeq operator

Two's complement is a method for representing signed integers in binary. Its algorithm, formulated as TwosComp, performs an XOR of each bit of input with 1 then adds 1 to the final bit sequence. $X O R$ is formulated as well; though TLA+ has a built-in XOR symbol, it only operates on Boolean values. The formula for BinaryAddl is a composition of other formulas that converts a binary sequence to a single decimal number, adds 1 , then converts back to a binary sequence. These three operators are shown in TLA+ Snippet 13. 


$$
\begin{aligned}
& \text { TwosComp }(\text { seq }) \triangleq \text { BinaryAdd } 1([x \in \operatorname{DOMAIN} \text { seq } \mapsto X O R(\operatorname{seq}[x], 1)]) \\
& \begin{array}{rl}
\text { XOR }(a, b) \triangleq C A S E & a=1 \wedge b=1 \rightarrow 0 \\
& \square a=1 \wedge b=0 \rightarrow 1 \\
& \square a=0 \wedge b=1 \rightarrow 1 \\
& \square \text { OTHER } \rightarrow 0
\end{array}
\end{aligned}
$$

$\operatorname{BinaryAdd1}(\mathrm{seq}) \triangleq \operatorname{DecimalToBinarySeq}(\operatorname{BinarySeqToDecimal}(\operatorname{seq})+1)$

TLA+ Snippet 13: The TwosComp and BinaryAdd1 operators

The TLA+ formula for calculating the LRC of a sequence, CalculateLRC, can thus be written as a composition of the other formulas in TLA+ Snippet 14. The

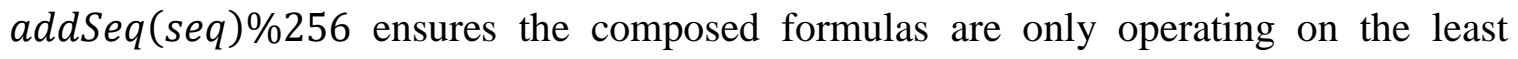
significant byte of the sum of all the bytes.

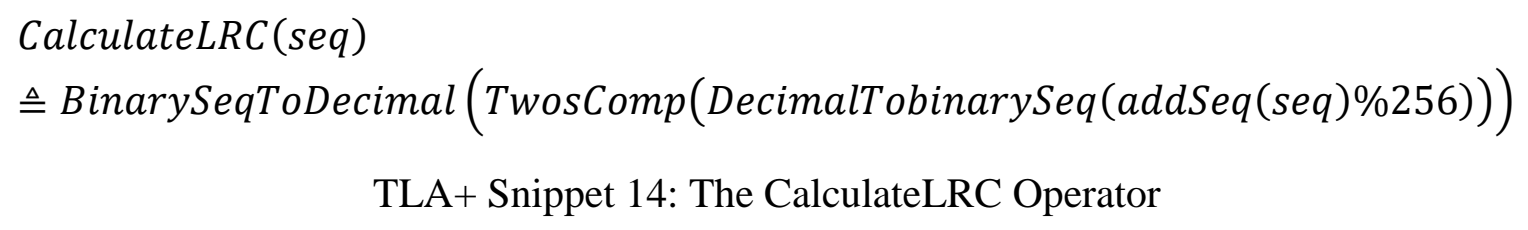

\subsection{Summary}

This chapter presented the formalized specification in TLA+ for the field device security preprocessor and the formalized security and safety properties required to check the correctness of the specification. Each thread within each component of the preprocessor is defined with its own formal specification and its own security properties. After each component is finalized, it is composed into a single specification for the security preprocessor and new properties that could not be checked in individual components are formalized for checking. Additionally, some helper functions that were used during the research and are novel formal specification in TLA+ are presented. The next chapter will discuss the model checking strategies used to reduce the state space and the results produced by the TLC checker. 


\section{CHAPTER V}

\section{MODEL CHECKING, INPUT VALUES, STATES}

\subsection{Introduction}

Checking a TLA+ specification involves using the TLC model checker to generate a state-space and exhaust that space looking for violations of properties. This chapter describes the checking statistics produced by TLA+ and the design strategies employed to reduce the size of the state-space and create models that are feasible to check. All properties described in Chapter 0 are checked for their respective specifications. While each TLA+ design required multiple revisions and intensive thought to build, the stats and results presented in this chapter are only for the final iteration wherein all properties were successfully validated and all states were visited at least once. Most of the designs were checked quickly, around 10 seconds when not looking checking temporal properties and roughly 10 minutes when temporal properties are enabled.

\subsection{TLC Model Checker}

Temporal Logic of Actions, and the TLA+ language, are expressive tools for specifying concurrent distributed systems using the notation of mathematics and logic. The expressiveness of the TLA+ language is intentionally designed with formal reasoning on complex specifications in mind, not for mechanically checking those specifications. TLA+ allows specifying a system with an undefined number of 
processors, unbounded message queues, and other potentially infinite constructs that do not cooperate with exhaustive checking. Model checking a system specified in TLA+ thus involves designing a finite-state model from the TLA+ specification - forgoing some of the undefined characteristics to search for simple design-level logic bugs.

If model checking is the goal then the selection of TLA+, a language that is too expressive to be directly checked, might raise some questions. Model checking a formal specification can be considered a steppingstone to an eventual goal of a proof of correctness. Yuan Yu et al set forth the motivations for creating a mechanical checker for $\mathrm{TLA}+$ in [142]. Their motivations are summarized here:

1 Allow design of a complex system in an expressive language like TLA+ and check a finite-state model ${ }^{6}$ of that design to catch bugs before proof work.

2 Allow designers to check their design while developing it without translation into a separate, less expressive language removing the complications that could arise from such a translation.

A TLA+ specification is expressive enough to allow formal proofs. The TLC model checker can thus be thought of as a proof aid in allowing designs to be easily checked for logical inconsistencies before investing time in theorem proving. A specification can be as expressive as needed but contain parameters to limit the specification at time of checking.

${ }^{6}$ A specification models a design if it meets the requirements of that design. In this case, a model of a model is checked. 


\subsection{State Explosion Considerations}

Exponential increase in state-space, referred to as state explosion, is a driving force in model checking projects and research [80]. The work presented here includes a variety of considerations that could drastically affect the runtime of TLC. The most prominent consideration is the selection of Modbus messages that are passed between components in this system, both in number and in branching techniques. Firstly, the number of messages that are passed in the specification increases the size of the state space as a new set of states it generated for each new message. Secondly, the way the successive messages are handled can drastically affect the rate of growth of the state space. A specification that is designed to process every message in a queue within a single trace enjoys a tragic fall into the depths of computational complexity as each successive message compounds the state-space generated from processing the previous messages. Alternatively, a specification that is designed to generate a new trace for each message will check the processing of each message independently. The difference in these two scenarios can be boiled down to the selection of either Equation (13) or Equation (14) where messages represents the queue of messages waiting to be processed by the system and testmessages is a set or ordered sequence (depending on the needs of the specification) of relevant messages with which to test the model. A state-space is complete when all messages have been processed.

messages $=$ testmessages
messages $\in$ testmessages

Assuming two models are equivalent but for the value of messages in the initial state, Equation (13) was found to produce an exponentially larger state-space in practice. 
Equation (13) assigns to messages the entire set or sequence testmessages. In the threadlevel specifications found in this research, once a message is fully processed the thread checks messages for another message to process. If messages contains more than one element, a state-space for the successor message must be initiated from every state the system could find itself in after completing the predecessor message resulting in multiple traces for a single message. If there are more messages, this process repeats for each ending state of each trace of the previous message. Figure 29 shows the development of such a state-space. TLC includes some tricks behind the scenes to prevent redundant state generation where it can be detected. Even with optimization, Equation (13) produces a narrower, deeper tree of states. TLC generates and checks new states using a breadth-first search so a deep and narrow tree could result in property violations being discovered later. Further discussion can be found in [64].

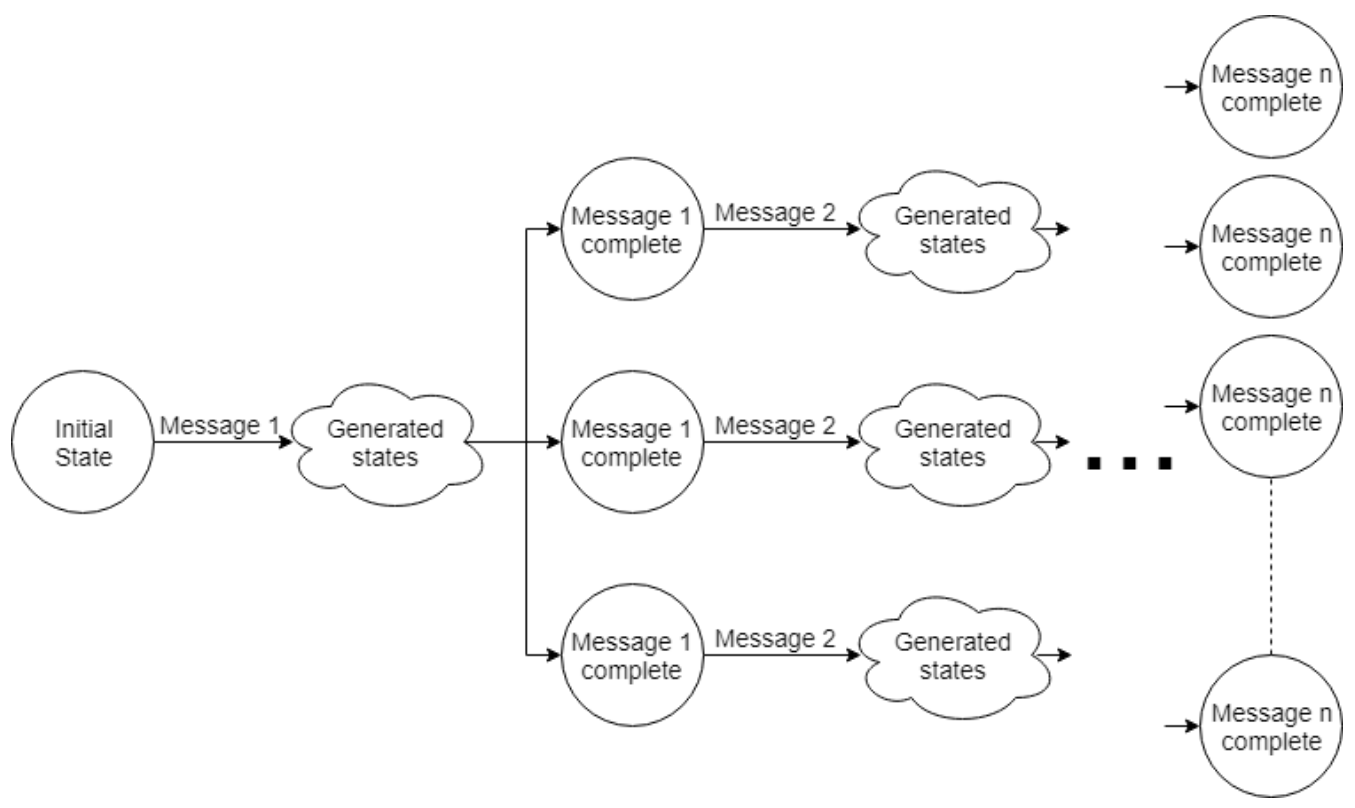

Figure 29: The state space generated from Equation (13).

Where appropriate, Equation (14) is used to give messages a value in the initial state. This definition tells TLC that messages could hold any value that is an element of 
testmessages, so TLC branches and creates a separate trace for each possible value of messages. Practically, this means a separate state-trace is created for each message in testmessages and is terminated at the end of that message's processing. Figure 30 shows the state-space for this method. This figure also demonstrates an obvious path for parallelization of the state generation and checking as each next-state after the initial can be handed off to another process.

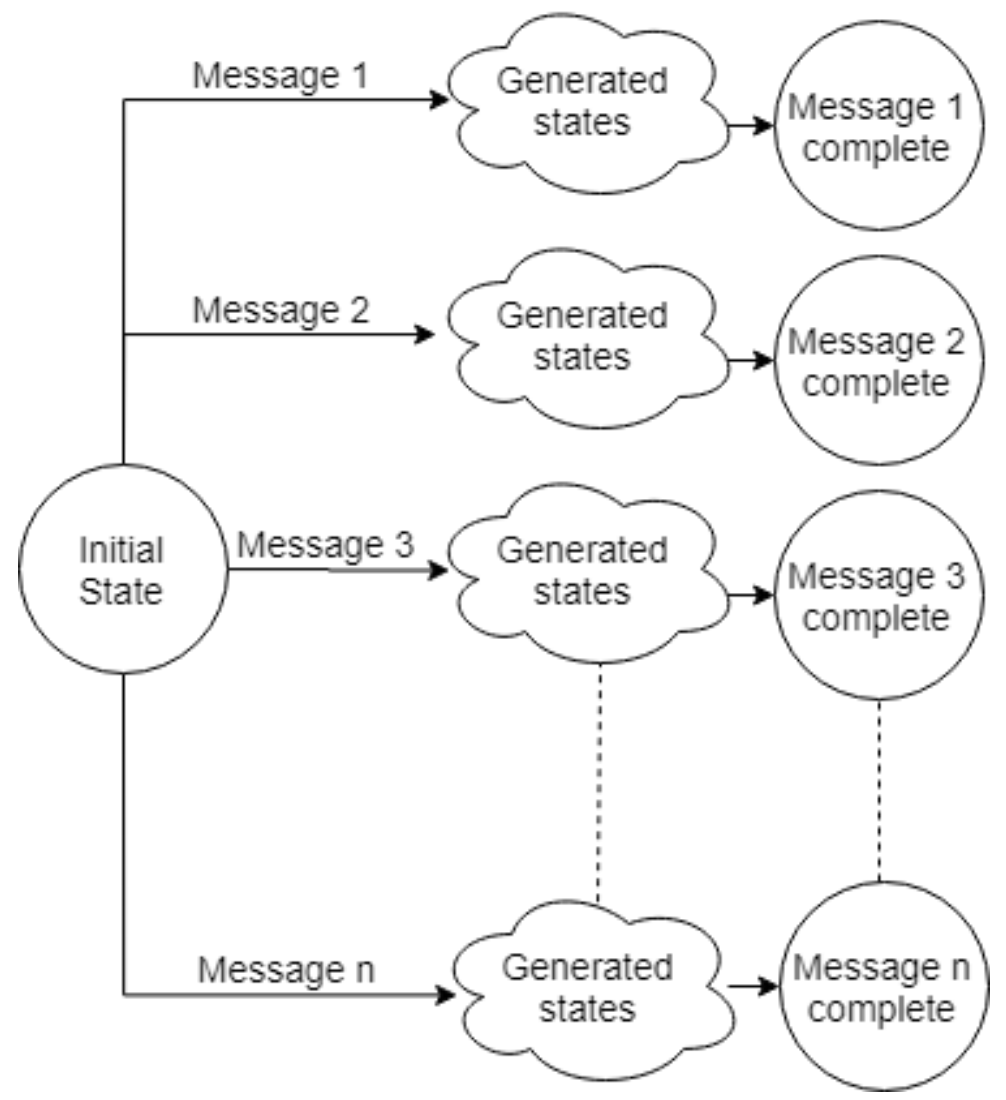

Figure 30: The state space generated from Equation (14.

Another consideration for handling the state explosion problem in this work is the type of properties to be checked. Invariants, properties that must always be true, are quickly checked upon generation of each state. Temporal properties are difficult and slow because the path a given trace took through the state-space matters. For temporal property $Q$, TLC must check if there exists a path through the state space that does not contain a 
state that satisfies $Q$. All paths must be checked to satisfy temporal properties. As much of the checking for temporal requirements for the field device is pushed into the individual thread models as possible as these are small. The few temporal properties that could not be avoided are checked over several different models (the same specification but with different initial values) to check sections of the state-space individually. At time of writing, TLC does not offer strong support for parallelization of temporal property checking [101].

\subsection{Trusted Network Component States and Inputs}

The trusted network component is the network interface that communicates with a PLC or other ICS actor within a physically protected process network. It is considered "trusted" because messages that are received from this network are assumed to be goodintentioned (though not necessarily well-formed). Two threads make up the trusted network component - Trustnet_in that receives messages from the network and passes them to inner components, and Trustnet_out that receives messages from the inner components and relays them to the process network.

The behavior of the Trustnet_in thread should not depend on any specific sequence of well-formed Modbus messages. Once a well-formed message has been completely processed, Trustnet_in should return to its initial state and no well-formed message should affect any successive messages. This allows use of Equation (14) in defining the set of messages to be processed in the initial state, meaning a separate statespace can be calculated for each test message quickly and concurrently. The behavior of Trustnet_out is potentially different from message to message so it is appropriate to specify a sequence of test messages using Equation (13). This thread operates on input 
from the inner components, so its input is complete messages rather than bytes. Operating on complete messages allows a significant reduction in the state-space generated by TLC. Processing a message byte-by-byte requires loops and additional steps. Processing an entire message at once allows the specification to be greatly simplified. State-space statistics for Trustnet_in and Trustnet_out can be seen in Table 22: TLC Running Statistics for the trusted network component. Time is the wall time required to build and check the model. Diameter is the length of the longest state-trace.

Table 22: TLC Running Statistics for the trusted network component

\begin{tabular}{|l|l|l|l|l|}
\hline Spec & Time & Diameter & States Found & Distinct States \\
\hline trustnet_in & $0: 07$ & 6609 & 10037 & 9774 \\
\hline trustnet_out & $0: 06$ & 30 & 31 & 30 \\
\hline
\end{tabular}

The test messages crafted for Trustnet_in were a collection of malformed Modbus messages, a collection of well-formed Modbus messages, and every possible single-byte value that could cross the serial port (0-255). The selected test messages can be found in Table 23. These messages were translated into ACII hex before use in the model. Trustnet_out required structures for testing as the messages it received included the critical decision from inner components and message IDs. The test structures can be found in the appendix.

Table 23: Test messages for Trustnet_in

\begin{tabular}{|c|c|}
\hline Message & Purpose \\
\hline :JGP9432J39JGWIRW & Improper message with proper start \\
\hline$:\langle\mid r\rangle\langle\langle n\rangle$ & $\begin{array}{l}\text { Empty message with proper start and } \\
\text { termination }\end{array}$ \\
\hline JGP9432J39JGWIRW $<|r\rangle<\backslash n>$ & $\begin{array}{l}\text { Improper message with proper } \\
\text { termination }\end{array}$ \\
\hline$: 1103006 \mathrm{~B} 00037 \mathrm{E}<|\mathrm{r}\rangle\langle\operatorname{|n}\rangle$ & Well-formed message \\
\hline $\begin{array}{l}: 1103006 \mathrm{~B} 00037 \mathrm{ECRL103006B00037ECRL10300} \\
\text { 6B00037ECRL103006B00037ECRL103006B0003 }\end{array}$ & $\begin{array}{l}\text { Improper message, for troubleshooting } \\
\text { model first and design second. }\end{array}$ \\
\hline
\end{tabular}




\begin{abstract}
7ECRL103006B00037ECRL103006B00037ECRL 103006B00037ECRL103006B00037ECRL103006 B00037ECRL103006B00037ECRL103006B00037 ECRL103006B00037ECRL103006B00037ECRL1 03006B00037ECRL103006B00037ECRL103006B 00037ECRL103006B00037ECRL103006B00037E CRL103006B00037ECRL103006B00037ECRL10 3006B00037ECRL103006B00037ECRL103006B0 0037ECRL103006B00037ECRL103006B00037EC RL103006B00037ECRL103006B00037ECRL1030 06B00037ECR1103006B00037ECRL103006B000 37ECRL103006B00037ECRL103006B00037ECR L103006B00037ECRL103006B00037ECRL10300 6B00037ECRL103006B00037ECRL103006B0003 7ECRL103006B00037ECRL103006B00037ECRL 103006B00037ECRL103006B00037ECRL103006 B00037ECRL103006B00037ECRL103006B00037 ECRL103006B00037ECRL103006B00037ECRL1 03006B00037ECRL103006B00037ECRL103006B 00037ECRL103006B00037ECRL103006B00037E CRL103006B00037ECRL103006B00037ECRL10 3006B00037ECRL103006B00037ECRL103006B0 0037ECRL103006B00037ECGLF :1103006B000:1103006B00037E $<|r|<\backslash n$
\end{abstract}

$0,1,2,3, \ldots 254,255$
Well-formed message interrupts previous well-formed message All possible bytes, 0-255

\subsection{Untrusted Network Component States and Inputs}

Untrustnet_in is similar to Trustnet_in. It operates on bytes rather than whole messages and its state is unaffected by well-formed encapsulated messages. TLC found more states for Untrustnet_in than Trustnet_in because of the additional constraint of picking the well-formed Modbus message out of the encapsulated data that is received from the serial port. Trustnet_out operates on full messages rather than bytes so its specification generates much fewer states. TLC's state statistics for the untrusted network component can be found in Table 24. Test messages and structures for these specs can be found in the appendix. 
Table 24: TLC Running Statistics for the untrusted network component

\begin{tabular}{|l|l|l|l|l|}
\hline Spec & Time & Diameter & States Found & Distinct States \\
\hline Trustnet_in & $0: 09$ & 12015 & 16532 & 16269 \\
\hline Trustnet_out & $0: 09$ & 58 & 59 & 58 \\
\hline
\end{tabular}

\subsection{Protocol Checking States and Inputs}

The protocol checking state-space is the simplest of those described here. Its diameter, that is the longest useful trace through the state-space found by TLC, is only five states wide. This is likely because the protocol checking spec simply checks messages against a definition of Modbus before forwarding them. The bulk of the specification work occurs in the definitions of Modbus and its helper functions, but these would only generate transition rules between states. This component trusts the input from the other components, so the test structures were crafted to exhaust the definition of Modbus that this component is checking against. TLC's statistics for this component's single thread can be found in Table 25 and the test messages can be found with the specification in the appendix.

Table 25: TLC Running Statistics for the protocol checking component

\begin{tabular}{|c|c|c|c|}
\hline Time & Diameter & States Found & Distinct States \\
\hline $0: 10$ & 5 & 24 & 20 \\
\hline
\end{tabular}

\subsection{Cryptographic Component States and Inputs}

The cryptographic component produces another relatively simple state-space compared to the networking components. Like the protocol checker, the cryptographic component's complexity comes from its transition rules and definitions. The cryptographic checking additionally benefits from the abstraction of cryptographic function, reducing the state-space and complexity. A hash value is hard coded within the 
model - no calculations are performed. TLC's statistics for Sign and Verify can be found in Table 26 and the test messages can be found with the specification in the appendix.

Table 26: TLC Running Statistics for the cryptographic component

\begin{tabular}{|l|l|l|l|l|}
\hline spec & Time & Diameter & States Found & Distinct States \\
\hline sign & $0: 06$ & 14 & 15 & 14 \\
\hline verify & $0: 05$ & 18 & 38 & 38 \\
\hline
\end{tabular}

\subsection{System Model States and Inputs}

Checking the model of the entire system presented the largest challenge as ensuring the properties for the individual models were preserved often clashed with abstracting already-proved pieces to reduce the complexity of the composite. The composite model is where the majority of the strategies for controlling the state-space growth came into play. This is the only model that exercises the concurrent modeling abilities of TLA+. The extra complexity of undefined execution order of concurrent states was made evident in the drastically higher running statistics produced by TLC in Table 27.

Table 27: TLC Running Statistics for the security preprocessor

\begin{tabular}{|l|l|l|l|l|}
\hline $\begin{array}{l}\text { spec Time } \\
\begin{array}{l}\text { Composite w/ } \\
\text { trustnet input }\end{array}\end{array}$ & $31: 11: 07$ & 7822 & $946,531,170$ & $221,952,298$ \\
\hline $\begin{array}{l}\text { composite w/ } \\
\text { untrustnet input }\end{array}$ & $31: 08: 01$ & 7821 & $942,382,213$ & $221,005,455$ \\
\hline
\end{tabular}

Checking had to be divided across multiple runs with different inputs to networking components. Input to the model was designed such that every state was visited at least once across the runs. In the first run, the test messages were placed into the queue of the trusted networking component while the queue of the untrusted networking component was left empty. In the second run, test messages were placed into 
the queue of the untrusted networking component while the queue of the trusted networking component was left empty. Time was also saved by checking all the invariant properties first, then checking the temporal properties one at a time across different runs. While this may not have saved computation time, it did reduce the time between runs during development when changes to the properties and specification were frequent.

\subsection{Summary}

The process of checking a TLA+ model is simple: configure the properties to check then run TLC. Writing a model and properties to be checked efficiently can be difficult and time-consuming. This chapter detailed the statistics from the TLC model checker when run on the completed models presented in Chapter 0. These statistics are for the runs in which TLC did not find any property violations after an exhaustive search. The individual threads were quick and easy to check by design, but they were not enough to validate all the desired properties. Validating the remaining properties on the larger model was a greater task that often required days of computation. When taken together, these runs offer proof that the properties this thesis set out to prove hold for every state the security preprocessor could find itself it. 


\section{CHAPTER VI}

\section{CAMKES ARCHITECTURE FOR A BUMP-IN-THE-WIRE SECURITY PREPROCESSOR}

\subsection{Introduction}

Designing an embedded system with security in mind often has competing goals. Embedded systems are generally low on processing power and memory which limits the functionality that can be supported, especially in time-sensitive environments [2], [17], [104]. Simplifying the design can help, as well as working from a microkernel that adds a lot of security and safety features natively. CAmkES is the architecture design framework for building native seL4 applications. A native seL4 application, as opposed to an application that would run in a Windows or Linux virtual machine on top of seL4, is built to take advantage of the security and safety features the seL4 microkernel provides. A virtualized instance of Windows or Linux would add millions of lines of unnecessary and unsafe code to an embedded device, potentially expanding the attack surface. However, a native seL4 application that runs directly on top of seL4 could have its critical components isolated. Isolation not only add layers of security within the native application, but also eliminates categories of vulnerabilities common in less reliable applications and microkernels such as memory violations and pivoting malware. This chapter presents the CAmkES definitions for each component and connection that was formally specified in TLA+ in Chapter $\mathrm{x}$ for a native seL4 control system security $\begin{array}{llll}\text { preprocessor } & \text { (SPP). } & \text { This demonstrates }\end{array}$ 
the implementation portion of the developments steps proposed in Chapter $\mathrm{x}$ and refreshed in Figure 31.

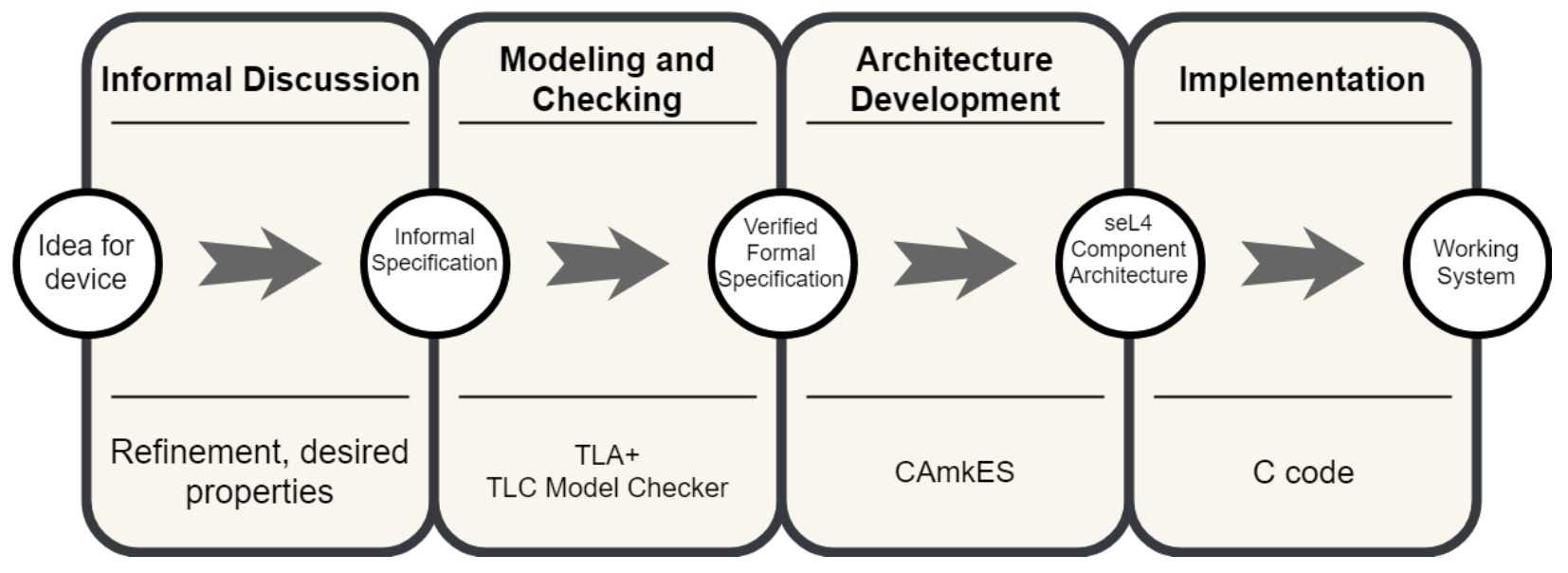

Figure 31: Development steps for verifying seL4 designs using TLA+.

\subsection{CAmkES Definitions for Components, Interfaces, and Connections}

The work presented here leverages the isolation guarantees provided by the seL4 microkernel and carried through the automatic code generation of the CAmkES framework. There are four components described in this section: a cryptographic service provider, a Modbus protocol checker, a network interface for the trusted network, and a network interface for the untrusted network. Figure 32 illustrates the components and their connections. The blue outline of two components indicate they are active, meaning they have a thread of control. The other two components are passive, meaning they only provide a service and must be called by an active component before any work is done. Table 28 shows the relationship between CAmkES components and TLA+ specifications described in the Chapter 0. Some of the components have multiple specifications, one for each thread of execution within the component. The remainder of this section describes each component and each connection in more detail. 


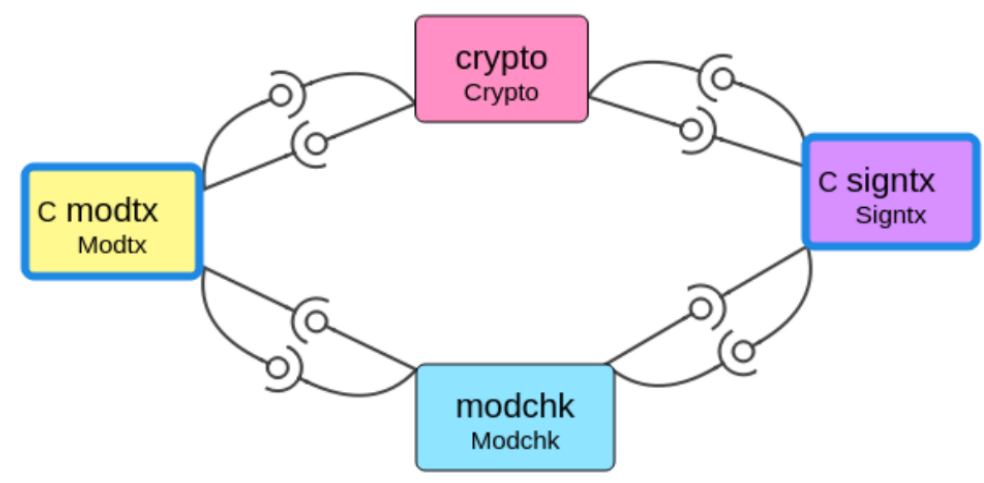

Figure 32: CAmkES output for the system architecture

Table 28: Relationship between TLA+ specifications and CAmkES components

\begin{tabular}{|l|l|}
\hline CAmkES Component & TLA+Specification \\
\hline Modtx & Trustnet_in \\
\hline Crypto & Trustnet_out \\
\hline Modchk & Sign \\
\hline Signtx & Verify \\
\hline
\end{tabular}

\subsubsection{Modtx: The Trusted Network Interface}

The trusted network is the control system intranet, or process network. In the control center this means the HMI, the PLC, the engineers and operators, the data historian, and any control equipment required within the confines of the building. In a substation, the trusted network includes the remote terminal unit and any cyber-physical instrumentation required for the mission. The trusted network utilizes SCADA network protocols, in this case Modbus. The purpose of the trusted network interface is to read bytes from the serial port and pass well-formed messages to the inner components with a generated ID. Note that well-formed does not mean valid. Modtx simply looks for the ":" character that indicates the start of a Modbus message and the " $/ \mathrm{r} / \mathrm{n}$ " sequence that 
indicates a finished message and ensures that the message is an appropriate length. Message validation is left to the inner components.

Modtx is also responsible for sending well-formed, valid Modbus messages out of the serial port onto the trusted network. This functionality is a bit more complex as the validation of a Modbus message requires agreement from two other components working asynchronously. When a message is received from an inner component, it is stored until its counterpart (indicated by a message ID) is received from the other component. If both components agree that the message is valid, it is sent byte-by-byte through the serial port. If one or both inner components indicate the message is invalid, Modtx drops the message. This design facilitates proofs that only well-formed, valid Modbus can be sent to the trusted network. Figure 33 illustrates the flow of messages through the Modtx component.

The code in Figure 34 shows the definition for the Modtx component. As it is responsible for polling the serial interface, line 9 designates this as an active component. It provides a single interface called ModtxIface that provides the message compiling and sending service. It consumes two interfaces, one for each inner component, that allow Modtx to forward a message through the system. The code in Figure 35 shows the definition for Modtx's interface. The lone function, print, is meant to be consumed by the inner components. It takes as parameters the contents of the Modbus message, the component from which the RPC originated, the ID of the message, and finally the decision of the inner component on whether the message is valid. 


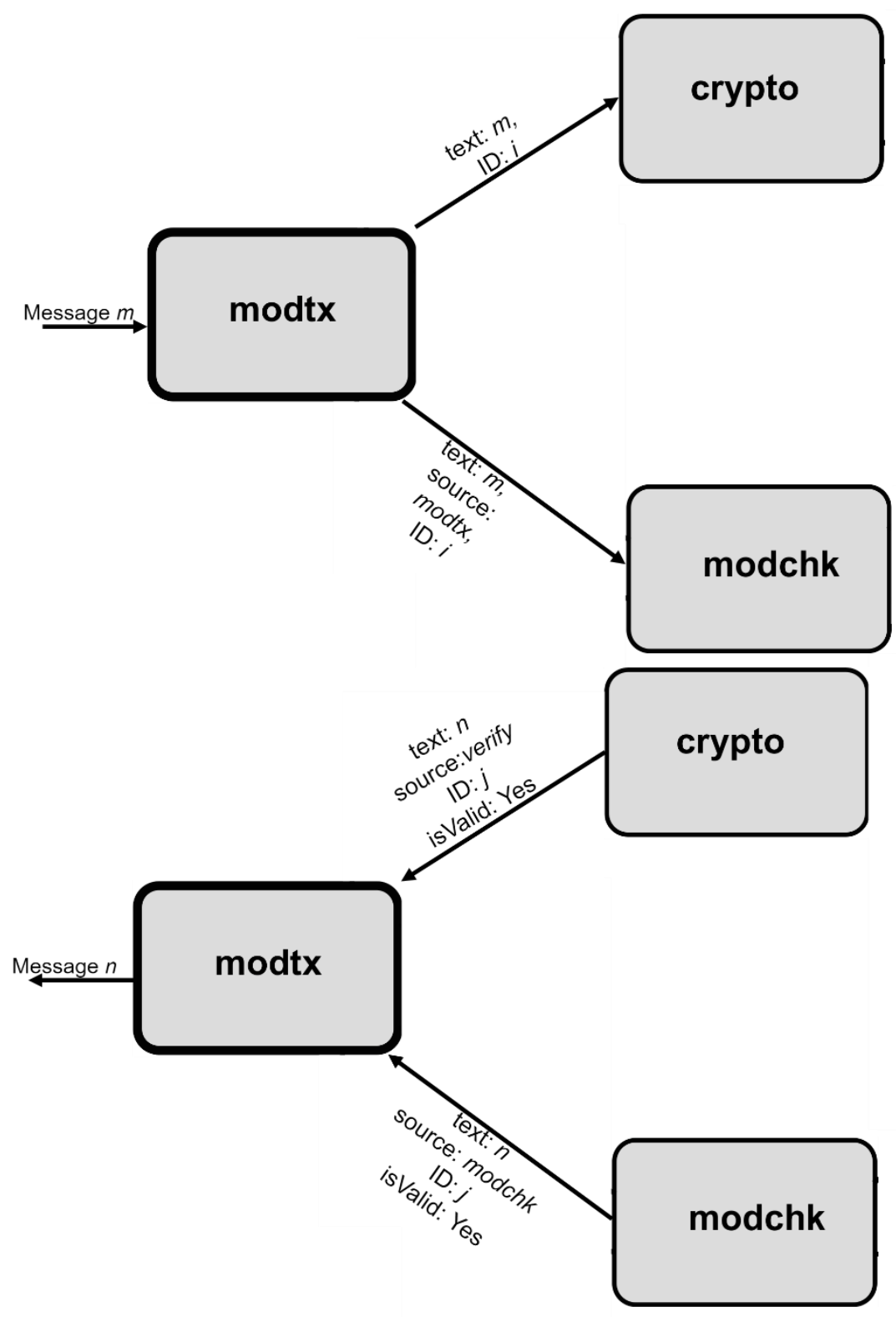

Figure 33: Flow of messages through Modtx

\subsubsection{Signtx: The Untrusted Network Interface}

The untrusted network is anything outside the control network. In this specific case, it is the connection from the control center to the substation. This medium can vary from installation to installation, and might use network infrastructure that is not within 
the operator's control such as a telephone lines. The protocols used over the untrusted network will also vary depending on the installation. The system described in this section will send and receive an encapsulated Modbus packet over a serial line to and from a modem. In a similar fashion to Modtx described in section 6.2.1, the Signtx component is responsible for reading well-formed (not necessarily valid) encapsulated messages from the serial port, assigning an ID, and forwarding the message to the inner components. A well-formed message starts with a "!" character, ends with a " $/ \mathrm{r} / \mathrm{n}$ " sequence of characters, and is between 78 and 578 bytes long. Message validation is left to the inner components.

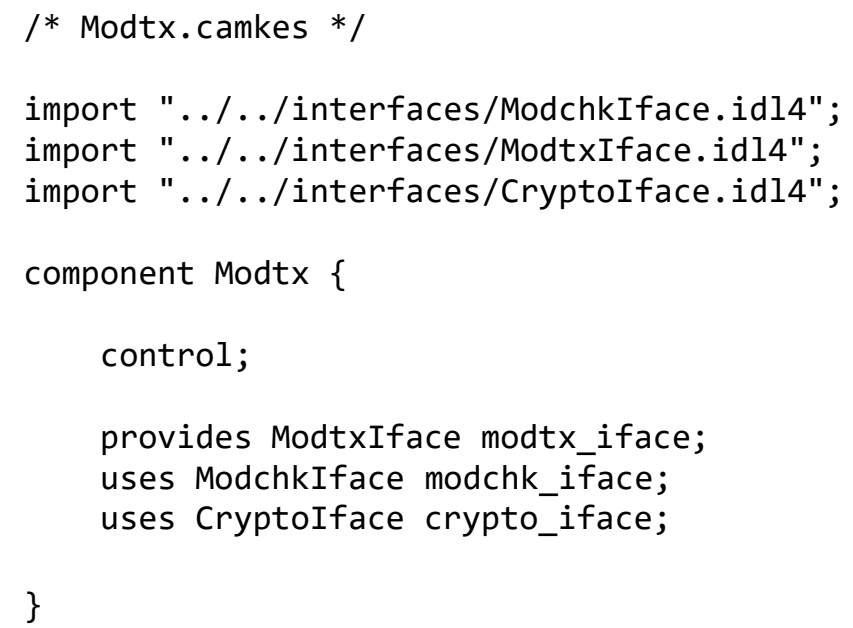

Figure 34: The Modtx component definition

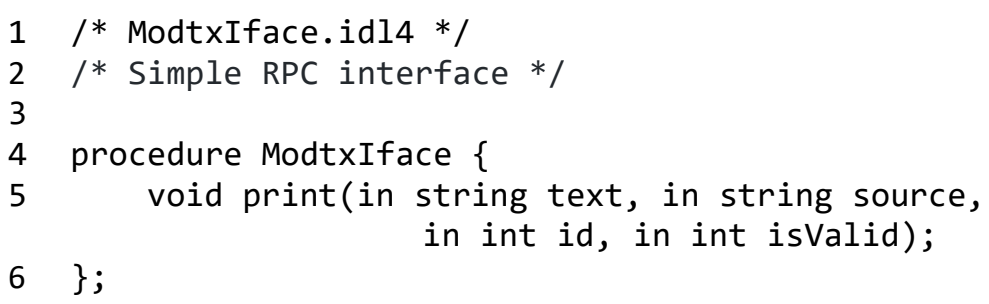

Figure 35: The ModtxIface interface definition 
The Signtx component is also responsible for sending well-formed, valid, signed encapsulated messages out of the serial port onto the untrusted network. This functionality is a bit more complex as the signing and validation of the Modbus message requires actions from two other components working asynchronously. When a message is received from an inner component, it is stored until its counterpart (indicated by a message ID) is received from the other component. If the protocol checker identifies the Modbus as valid and the signature has been received from the inner components, the final encapsulated message is sent byte-by-byte through the serial port. If the protocol checker decides the Modbus message is invalid, Signtx drops the message. This design facilitates proofs that only well-formed, valid, and signed encapsulated Modbus can be sent to the untrusted network. Figure 36 illustrates the message flow through Signtx.

The code in Figure 37 shows the definition for the Signtx component. As it is responsible for polling the serial interface, line 9 designates this as an active component. It provides a single interface called SigntxIface that initiates the message compiling and sending service. It consumes two interfaces, one for each inner component, that allow Signtx to forward a message and signature through the system. The code in Figure 38 shows the definition for Signtx's interface. There is a function within the interface for each of the inner components. The first, print_sign, is meant to be used by the cryptographic component to pass the cryptographic hashes of messages. It accepts as parameters the contents of the message, the source from which this RPC originated, the ID of the message, and the calculated HMAC. The second function of the interface, print_mod, is meant to be used by the protocol checker. It accepts as parameters the 
contents of the message, the source from which the RPC originated, the ID of the message, and the protocol checker's decision on whether the Modbus message is valid.

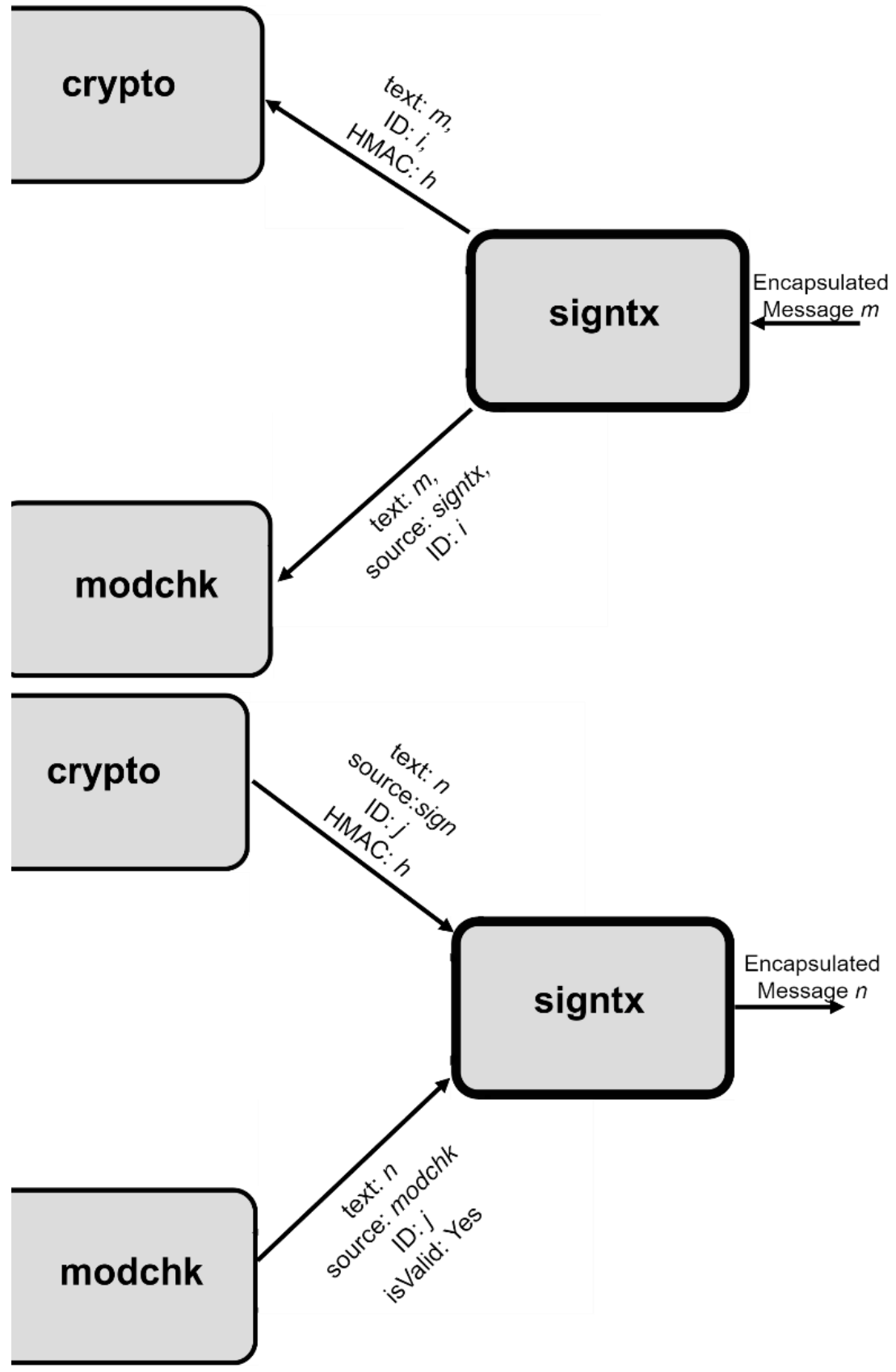

Figure 36: Flow of messages through Signtx 


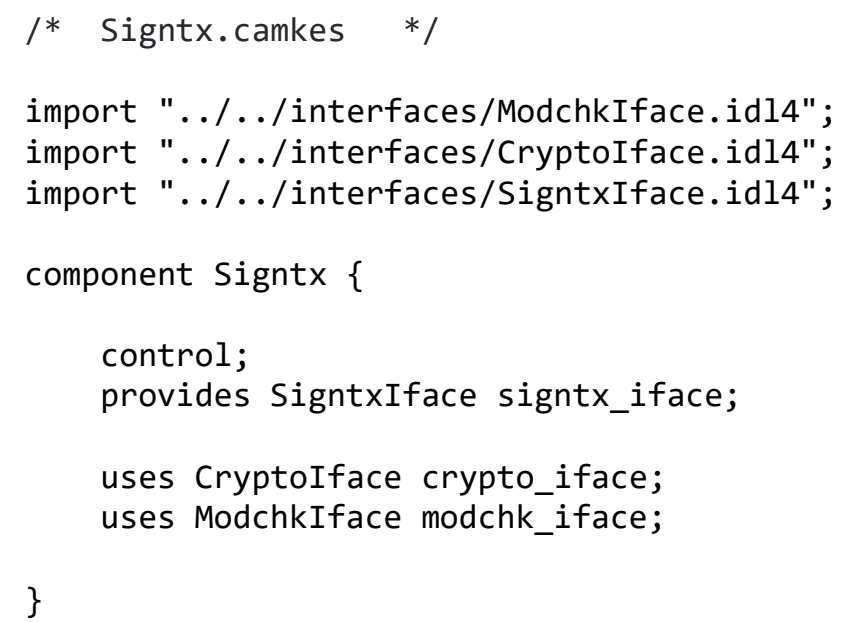

Figure 37: The Signtx component definition

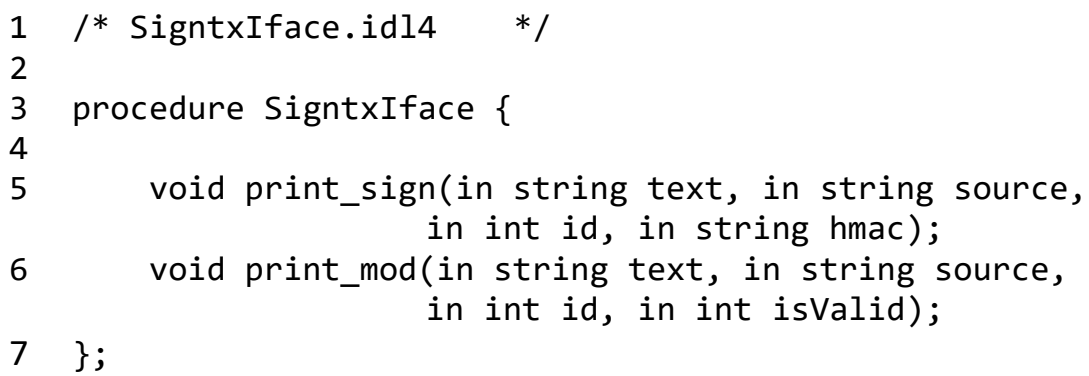

Figure 38: The SigntxIface interface definition

\subsubsection{Modchk: The Protocol Checker}

Modbus is an open standard communication protocol developed by Modicon in 1979. It is a simple and connectionless call-and-response protocol that allows straightforward modeling. This research deals specifically with Modbus ASCII, a version that uses a leading ":" to signal a new packet, contains two bytes to indicate the recipient's address, contains two bytes describing the function code, contains a payload of data, and finally a Longitudinal Redundancy Check (LRC) to detect transmission errors and an ending character sequence. It contains a simple addressing scheme allowing for up to 247 devices on a common bus, a field for a function code that tells the target devices which procedure to run, and a data field that can contain up to 252 bytes of information for the 
target device to act on. The layout of a Modbus packet can be seen in Table 29. Modbus ASCII in particular requires two bytes to represent one byte of information, thus the data field for Modbus ASCII has a maximum length of 504 instead of 252. Further reference about Modbus can be found in [29], while discussion of its use and security can be found in [21], [105].

Table 29: A Modbus ASCII message

\begin{tabular}{|c|c|c|c|c|c|c|}
\hline Start & Address & $\begin{array}{c}\text { Function } \\
\text { Code }\end{array}$ & Sub code & Data & LRC & End \\
\hline 66:" & 2 bytes & 2 bytes & $\begin{array}{l}2 \text { bytes } \\
\text { (optional) }\end{array}$ & $\begin{array}{c}\text { Up to } \\
504 \text { bytes }\end{array}$ & 2 bytes & "Ir In" \\
\hline
\end{tabular}

Modchk is an inner component responsible for checking the validity of Modbus messages it receives from either networking component. Messages it receives should already be well-formed, as the networking components only allow well-formed messages to pass. A valid Modbus message is a message in which every field conforms to the Modbus standard as described in [29]. This means the address field should contain a valid address from 0 (broadcast) to 247, the LRC matches a calculated LRC, and so forth. Once the message has been analyzed, it is forwarded to the opposite networking component with the critical decision attached.

The code in Figure 39 shows the definition for the Modchk component. This component merely provides a service and does not initiate any actions on its own, so it is passive. Modchk provides the same service regardless of the direction a message is passing through the system, so it can offer a single interface called ModchkIface. It consumes two interfaces, one from each networking component. When a message is received from Modtx, it is eventually forwarded using the Signtx interface and vice- 
versa. The ModchkIface is defined in Figure 40. It implements a single service: verify. Verify accepts as parameters the message text to be checked, the source component from which the RPC originated, and the ID of the message. The source allows Modchk to keep track of which component sent which message so the message can be forwarded appropriately.

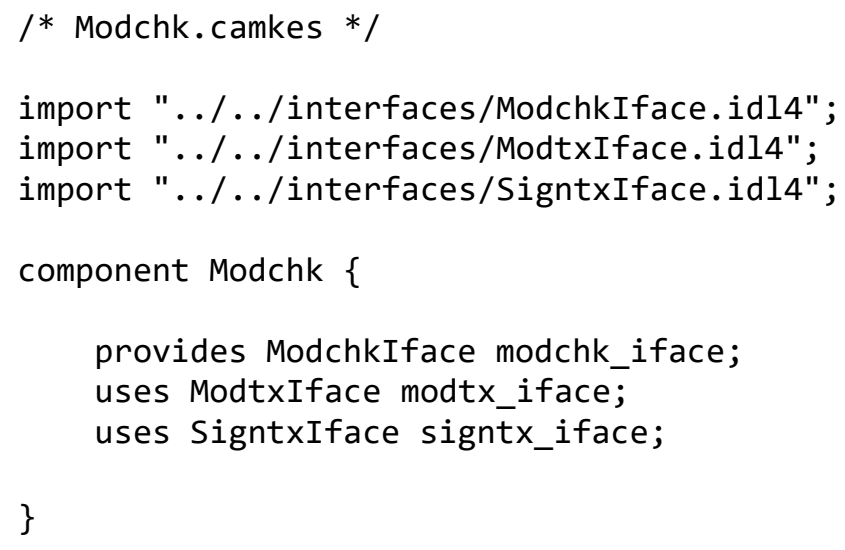

Figure 39: The Modchk component definition

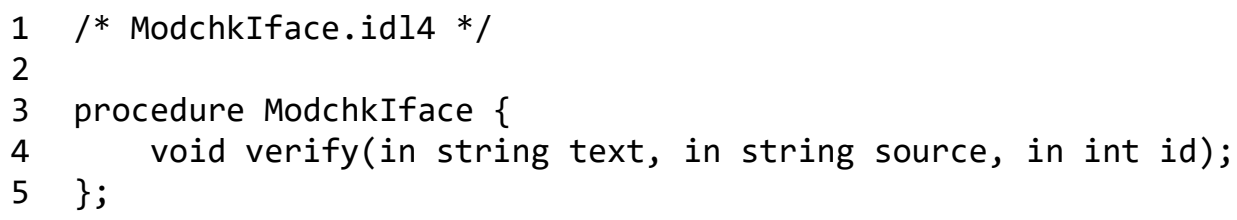

Figure 40: The ModchkIface interface definition

\subsubsection{Crypto: The Cryptographic Service}

The cryptographic component provides the signing and verifying services for the system. The goal of the system is to add authentication and integrity to an existing SCADA installation. To achieve that goal Crypto houses a secret key, a nonce generator, the cryptographic primitive SHA-256 cryptographic hashing function, and the cryptographic construction HMAC. Proper use of an HMAC can allow the system to detect if a message has been tampered with, as even the slightest change in the message 
will alter the resulting HMAC calculation. Additionally, because the secret key is included in the input to the HMAC, a message with a valid HMAC calculation can trusted to be from the opposite security device and not a forgery from an attacker.

The specific implementations of both SHA-256 and HMAC used in Crypto are verified to meet their respective FIPS specifications in [89] and [92]. The HMAC specification has been further verified to hold the security properties it claims in [143]. The verification work for these pieces of code can be seen in [144] and [88]. The properties have been proven to hold from the specification down to the $\mathrm{C}$ code that is found within Crypto. Currently, the default compiler for CAmkES and seL4 is the unverified gcc, so the binary that is produced from compilation of the $\mathrm{C}$ code must be trusted. Appel notes in [88] that gcc and the verified CompCert generally agree on language semantics, so the effort to verify the $\mathrm{C}$ code still adds value to the binary compiled by gcc.

The code in Figure 41 shows the definition for the Crypto component. This component merely provides a service and does not initiate any actions on its own, so it is passive. Crypto provides a single interface called CryptoIface that handles both the signing and verifying capabilities. It consumes two interfaces, one from each networking component. When a message is received from Modtx, it is eventually forwarded using the Signtx interface and vice-versa. The Crypto interface CryptoIface is defined in Figure 42. It implements two services: sign and verify. Sign is intended for consumption by the trusted network component, receiving raw Modbus messages and generating a cryptographic HMAC before forwarding the message and HMAC to the untrusted network component. It accepts as parameters the contents of a message and the message 
ID. Verify is intended for consumption by the untrusted network component, receiving a Modbus message and HMAC then calculating the HMAC itself and checking if the two HMACs match. It accepts as parameters the message contents, the ID of the message, and the HMAC that accompanied the message from the untrusted network. Once the cryptographic work has been done, both functions forward the results to the opposite network component.

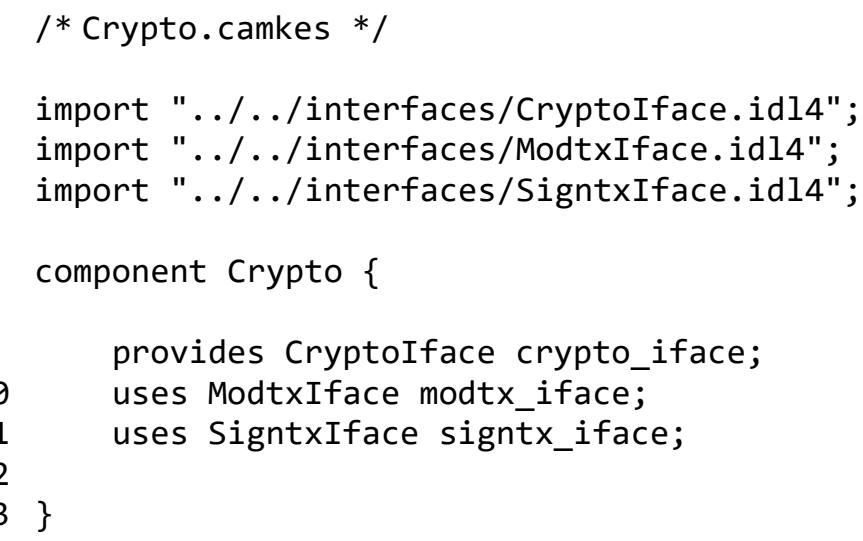

Figure 41: The Crypto component definition

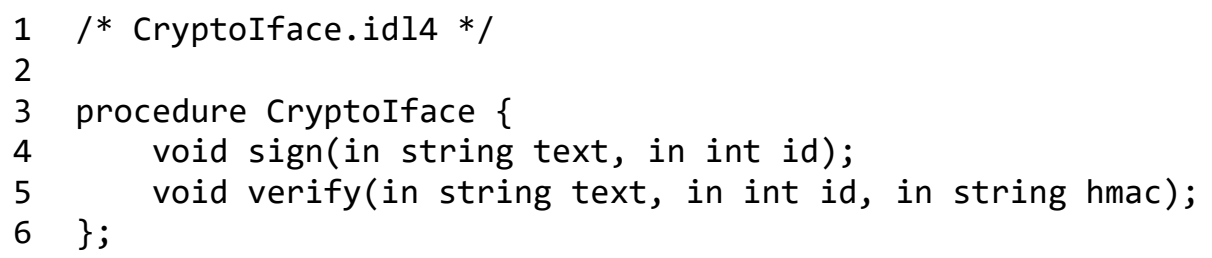

Figure 42: The CryptoIface interface definition

\subsubsection{Pre-defined RPC Connections}

With the components and interfaces defined, the last piece within CAmkES is to define the RPC paths that components can use to communicate with each other. These paths static and defined in the design before boot so they cannot be changed without 
recompiling the CAmkES application. The code snippet in Figure 43 defines the RPC calls from component interface to component interface, and create the lines and circles between components seen in Figure 32. An important feature of this design is the lack of allowed connections directly between the two networking components. Messages cannot flow through the system without going through the two inner components to be validated.

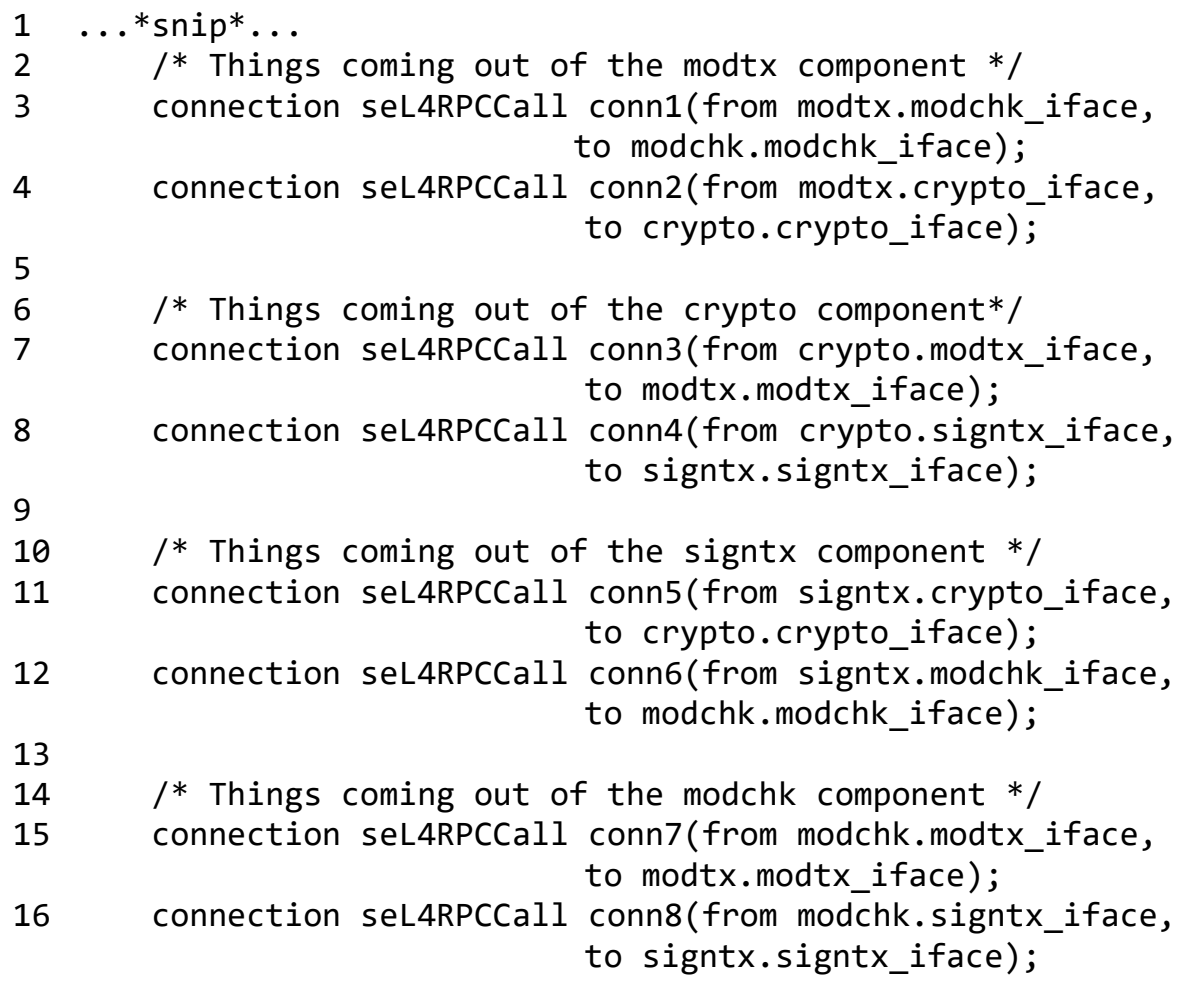

Figure 43: The system composition definition

\subsection{Summary}

This chapter detailed each CAmkES component for the native seL4 security preprocessor device. There are two passive inner components that handle critical decisions and two active outer components that interact with the network. The interfaces provided by each component are detailed, with the networking components providing interfaces with functions to print to the network and the inner components providing 
interfaces for the cryptographic and protocol checking services they provide. Finally, the seL4 RPC connections are detailed. The network components can communicate with the inner components but not with each other, no component can directly access functionality of another, and no sections of memory are shared between components. 


\section{CHAPTER VII}

\section{CONCLUSIONS AND FUTURE WORK}

The contributions presented in this dissertation fill a gap in the current state of the art research in securing industrial control and SCADA systems. This document describes the method for increasing the assurance and security level of a legacy control system though formal specification and model checking a bump-in-the-wire security preprocessor that adds much needed security mechanisms where none previously exists. Further, this dissertation serves as a proof of concept for the method of producing highassurance industrial devices by targeting the seL4 microprocessor with TLA+ designs. TLA + can be structured to intuitively flow from formally specified design to embedded CAmkES architecture running on seL4.

Adding security to an existing control systems network requires careful considerations to reduce downtime, reduce added latency, and reduce added failure points. A bump-in-the-wire security preprocessor built atop a high-assurance microkernel like seL4 might reduce the impact of added security enough to be palatable to asset

owners. This thesis proposed a development cycle for engineering high-assurance embedded systems with formally described and verified security and safety properties. An informal design of an embedded system can be formalized and verified using TLA+. The TLA+ specification can be used to define an architecture in CAmkES. Finally, the 
components in the CAmkES architecture can be populated with the $\mathrm{C}$ implementations of their algorithms.

Building trustworthy systems is a key component in both safety and security. The previous chapters have described a model of a bolt-on security device split into its integral pieces. Each state that each component can reach is described and automatically checked, demonstrating proof of relevant security properties. Building this system atop seL4 ensure that even though these components are proven to be isolated from each other, they are proven to be able to communicate with each other through highly specified channels. Thus this paper describes a system modeled and checked from end to end.

This work stops short of formally verifying an implementation of each cell specified in this paper. The described system takes advantage of seL4's distributed component architecture to show how a correct system should behave but does not describe its implementation. The initial steps of the next stage of research have been started to include a verified implementation, using Microsoft and INRIA's F* proof language[145][146][147] for the verification efforts then translating to $\mathrm{C}$ for use in each individual component. Verification of the implementation has not been attempted, but the pipeline of TLA+ to $\mathrm{F}^{*}$ to $\mathrm{C}$ source to compiled $\mathrm{C}$ within the CAmkES components has been shown to work with an implementation of a basic 4-component message passing system.

Another interesting avenue of research is a translation tool to automatically move from a TLA+ specification to a CAmkES architecture. This thesis performed the translation manually, but it might be possible to encode a subset of TLA+ semantics to 
generate the necessary directory structure, interface definitions, and CAmkES component definitions in an seL4 project. Processes in TLA+ might extract to components and message queues might extract to interface definitions. Such a tool will allow an engineer to specify, check, and reason about an seL4 architecture directly in TLA+. 


\section{REFERENCES}

[1] K. Stouffer, V. Pillitteri, M. Abrams, and A. Hahn, "Guide to Industrial Control Systems (ICS) Security.” US Department of Commerce, 2015.

[2] H. Mackenzie, "SCADA Security Basics: Why Industrial Networks are Different than IT Networks." Oct-2012.

[3] M. Abrams and J. Weiss, "Malicious Control System Cyber Security Attack Case Study - Maroochy Water Services, Australia," 2008.

[4] H. Kim, "Security and Vulnerability of SCADA Systems over IP-Based Wireless Sensor Networks," Int. J. Distrib. Sens. Networks, vol. 8, no. 11, p. 268478, 2012.

[5] V. M. Igure, S. A. Laughter, and R. D. Williams, "Security issues in SCADA networks," Comput. Secur., vol. 25, no. 7, pp. 498-506, 2006.

[6] N. Falliere, L. O. Murchu, and E. Chien, "W32.Stuxnet Dossier," 2011.

[7] I. N. Fovino, A. Carcano, M. Masera, and A. Trombetta, "Design and Implementation of a Secure Modbus Protocol," in Critical Infrastructure Protection III: Third Annual IFIP WG 11.10 International Conference on Critical Infrastructure Protection, Hanover, New Hampshire, USA, March 23-25, 2009, Revised Selected Papers, C. Palmer and S. Shenoi, Eds. Berlin, Heidelberg: Springer Berlin Heidelberg, 2009, pp. 83-96.

[8] "Network Infrastructure for EtherNet/IP: Introduction and Considerations." Open DeviceNet Vendor Association, Inc. (ODVA), 2007.

[9] B. Smith, "DNP3 Secure Authentication - What's all the Buzz about?".

[10] G. Klein et al., "seL4: Formal Verification of an OS Kernel." NICTA, 2009.

[11] R. Chapman, "Going Large with Formal Methods on iFACTS." 2014.

[12] E. M. Clarke and J. M. Wing, "Formal Methods: State of the Art and Future Directions," ACM Comput. Surv., vol. 28, no. 4, pp. 626-643, Dec. 1996.

[13] M. Rolfe, "How technology is transforming air traffic management," nats.aero, 2013. [Online]. Available: http://nats.aero/blog/2013/07/how-technology-istransforming-air-traffic-management/. [Accessed: 11-Dec-2016].

[14] A. Hall and R. Chapman, "Correctness by construction: developing a commercial secure system,” IEEE Softw., vol. 19, no. 1, pp. 18-25, 2002.

[15] D. Bailey and E. Wright, Practical SCADA for industry. Elsevier, 2003. 
[16] B. Galloway, G. P. Hancke, and others, "Introduction to industrial control networks.," IEEE Commun. Surv. Tutorials, vol. 15, no. 2, pp. 860-880, 2013.

[17] T. Brown, "Security in SCADA systems: how to handle the growing menace to process automation," Computing \& Control Engineering Journal, vol. 16, no. 3. pp. 42-47, 2005.

[18] R. M. Lee, SCADA And Me: A Book for Children and Management. IT-Harvest Press, 2013.

[19] J.-P. Thomesse, "Fieldbus technology in industrial automation," Proc. IEEE, vol. 93, no. 6, pp. 1073-1101, 2005.

[20] "Modbus." [Online]. Available: http://www.modbus.org/. [Accessed: 28-May2014].

[21] I. N. Fovino, A. Carcano, M. Masera, and A. Trombetta, "Design and Implementation of a Secure Modbus Protocol," in Critical Infrastructure Protection III: Third Annual IFIP WG 11.10 International Conference on Critical Infrastructure Protection, Hanover, New Hampshire, USA, March 23-25, 2009, Revised Selected Papers, C. Palmer and S. Shenoi, Eds. Berlin, Heidelberg: Springer Berlin Heidelberg, 2009, pp. 83-96.

[22] "MODBUS over Serial Line Specification \& Implementation guide V1.0," modbus.org, 2002. [Online]. Available: http://www.modbus.org/docs/Modbus_over_serial_line_V1.pdf. [Accessed: 05Aug-2018].

[23] G. R. Clarke, D. Reynders, and E. Wright, Practical modern SCADA protocols: DNP3, 60870.5 and related systems. Newnes, 2004.

[24] CONTROL MICROSYSTEMS, "DNP3 User and Reference Manual." Control Microsystems, 2007.

[25] P. Gibson, “Open and secure SCADA with DNP3.” Jun-2008.

[26] DNP Users Group, “A DNP3 Protocol Primer.” 2005.

[27] V. R. Schiffer Vangompel, "The common industrial protocol (CIP) and the family of CIP networks," ODVA, 2016.

[28] "Network Infrastructure for EtherNet/IP: Introduction and Considerations." Open DeviceNet Vendor Association, Inc. (ODVA), 2007.

[29] Modbus.org, "MODBUS over Serial Line Specification \& Implementation guide." Modbus.org, 2002.

[30] A. Miller, "Trends in Process Control Systems Security," IEEE Secur. Priv. Mag., vol. 3, no. 5, pp. 57-60, Sep. 2005.

[31] M. Brandle and M. Naedele, "Security for Process Control Systems an Overview," IEEE Security and Privacy, vol. 6, no. 6. IEEE, pp. 24-29, 2008. 
[32] S. Tom, D. Christiansen, and D. Berrett, "Recommended practice for patch management of control systems," 2008.

[33] K. Brocklehurst, "Cyberterrorists Seek to Cause Physical Harm.” Feb-2015.

[34] M. S. Branicky, S. M. Phillips, and W. Zhang, "Stability of networked control systems: Explicit analysis of delay," in American Control Conference, 2000. Proceedings of the 2000, 2000, vol. 4, pp. 2352-2357.

[35] F.-L. Lian, J. Moyne, and D. Tilbury, "Network design consideration for distributed control systems," IEEE Trans. Control Syst. Technol., vol. 10, no. 2, pp. 297-307, 2002.

[36] W. Johnson, R. R. Dunn, and V. J. Maggioli, "Understanding ISA84," Intech with Ind. Comput., vol. 59, no. 3, p. 12, 2012.

[37] J. H. Saltzer and M. D. Schroeder, "The protection of information in computer systems.," Proc. IEEE, vol. 63, no. 9, pp. 1278-1308, 1975.

[38] R. M. Lee, M. J. Assante, and T. Conway, "Analysis of the Cyber Attack on the Ukrainian Power Grid,” 2016.

[39] "Assessing Security and Privacy Controls in Federal Information Systems and Organizations," 2014.

[40] W. McGrew, "Rising to the Challenge of Pen Testing ICS," hornecyber.com, 2016. [Online]. Available: http://blog.hornecyber.com/attack-surface/rising-to-thechallenge-of-pen-testing-ics. [Accessed: 04-Dec-2018].

[41] D. Duggan, "Penetration Testing of Industrial Control Systems," 2005.

[42] R. Amoah, S. Camtepe, and E. Foo, "Formal modelling and analysis of DNP3 secure authentication," J. Netw. Comput. Appl., vol. 59, pp. 345-360, 2016.

[43] United Nations Office of Counter-Terrorism and CTED, "The protection of critical infrastructure against terrorist attacks: Compendium of good practices." INTERPOL, 2018.

[44] R. J. Anderson, Security engineering: a guide to building dependable distributed systems. John Wiley \& Sons, 2010.

[45] J. H. Saltzer, "Protection and the control of information sharing in Multics," Commun. ACM, vol. 17, no. 7, pp. 388-402, 1974.

[46] J. Wetzels, "Ghost in the Machine: Challenges in Embedded Binary Security," 2017.

[47] A. Wadsworth and B. Parker, "Implementing Application Whitelisting - A Case Study," in Noth American SANS SCADA \& Process Control System Security Summit, 2012.

[48] "Blue Coat ICS Protection Scanner Station Version," 2014.

[49] "UNIDIRECTIONAL SECURITY GATEWAYS," Waterfall-security.com. 
[Online]. Available: https://waterfall-security.com/unidirectional-securitygateways.

[50] Tofino Security, "Cyber Security for SCADA and Industrial Control Systems." [Online]. Available: http://www.tofinosecurity.com/. [Accessed: 08-Jun-2014].

[51] D. McMillen, "Security attacks on industrial control systems," 2017.

[52] N. Falliere, "Stuxnet Introduces the First Known Rootkit for Industrial Control System." Aug-2010.

[53] N. Anderson, "Confirmed: US and Israel created Stuxnet, lost control of it." Jun2012.

[54] R. Langner, "Stuxnet: Dissecting a Cyberwarfare Weapon," IEEE Secur. Priv. Mag., vol. 9, no. 3, pp. 49-51, May 2011.

[55] D. Wagner, "infrastructure under attack," Risk Manag., vol. 63, no. 8, p. 28, 2016.

[56] J. E. Sullivan and D. Kamensky, "How cyber-attacks in Ukraine show the vulnerability of the U.S. power grid," Electr. J., vol. 30, no. 3, pp. 30-35, 2017.

[57] U. Shamir, “Analyzing a New Variant of BlackEnergy 3.” SentinelOne, 2016.

[58] J. P. Anderson, “Computer Security Technology Planning Study,” ESDTR. 1972.

[59] D. E. Bell, "Looking back at the bell-La padula model," in Proceedings - Annual Computer Security Applications Conference, ACSAC, 2005.

[60] Department of Defense, "Trusted computer system evaluation criteria ["Orange Book"],” Dep. Def., 1985.

[61] B. Rudis, "CVE 100K: By The Numbers," Rapid7 Blog, 2018. [Online]. Available: $\quad$ https://blog.rapid7.com/2018/04/30/cve-100k-by-the-numbers/. [Accessed: 07-Oct-2019].

[62] L. Lamport, LATEX: a document preparation system: user's guide and reference manual. Addison-wesley, 1994.

[63] L. Lamport, "The temporal logic of actions," ACM Trans. Program. Lang. Syst., vol. 16, no. 3, pp. 872-923, 1994.

[64] L. Lamport, Specifying systems: the TLA+ language and tools for hardware and software engineers. Addison-Wesley Longman Publishing Co., Inc., 2002.

[65] T. Nipkow and G. Klein, "Concrete Semantics," A Proof Assist. Approach, 2014.

[66] P. Cousot, "Abstract interpretation based formal methods and future challenges," in Informatics, 2001, pp. 138-156.

[67] R. Milner, "A theory of type polymorphism in programming," J. Comput. Syst. Sci., vol. 17, no. 3, pp. 348-375, 1978.

[68] J. Spolsky, "Painless Functional Specifications," Joel on Software, 2000. [Online]. 
Available: $\quad$ http://www.joelonsoftware.com/articles/fog0000000036.html. [Accessed: 10-Oct-2016].

[69] E. M. Clarke, O. Grumberg, and D. Peled, Model checking. MIT press, 1999.

[70] K. L. McMillan, "Symbolic model checking," in Symbolic Model Checking, Springer, 1993, pp. 25-60.

[71] S. A. Kripke, "Semantical analysis of modal logic i normal modal propositional calculi," Math. Log. Q., vol. 9, no. 5-6, pp. 67-96, 1963.

[72] A. Pnueli, "The temporal logic of programs," in Foundations of Computer Science, 1977., 18th Annual Symposium on, 1977, pp. 46-57.

[73] O. Maler and D. Nickovic, "Monitoring Temporal Properties of Continuous Signals," 2011.

[74] M. Leuschel, "The ProB Animator and Model Checker," Heinrich-HeineUniversity, Institut für Software und Programmiersprachen, 2018. [Online]. Available: https://www3.hhu.de/stups/prob/index.php/Team. [Accessed: 07-Feb2018].

[75] R. E. Bryant, "Graph-based algorithms for boolean function manipulation," Comput. IEEE Trans., vol. 100, no. 8, pp. 677-691, 1986.

[76] A. Valmari, "Stubborn sets for reduced state space generation," in International Conference on Application and Theory of Petri Nets, 1989, pp. 491-515.

[77] G. J. Holzmann and D. Peled, "An improvement in formal verification," in Formal Description Techniques VII, Springer, 1995, pp. 197-211.

[78] P. Godefroid and P. Wolper, "A partial approach to model checking," Inf. Comput., vol. 110, no. 2, pp. 305-326, 1994.

[79] E. Clarke, A. Biere, R. Raimi, and Y. Zhu, "Bounded model checking using satisfiability solving," Form. methods Syst. Des., vol. 19, no. 1, pp. 7-34, 2001.

[80] E. Clarke, O. Grumberg, S. Jha, Y. Lu, and H. Veith, "Progress on the state explosion problem in model checking," in Informatics, 2001, pp. 176-194.

[81] USGAO, "National Partnership Offers Benefits, but Faces Considerable Challenges," 2006.

[82] "CommonCriteria," commoncriteriaportal.org, 2018. [Online]. Available: https://www.commoncriteriaportal.org/. [Accessed: 04-Dec-2018].

[83] Common Criteria, "Common Criteria," 2017. .

[84] Common Criteria, "Certified Products," 2017. [Online]. Available: https://www.commoncriteriaportal.org/products/.

[85] L. A. Johnson and others, "DO-178B, Software considerations in airborne systems and equipment certification," Crosstalk, Oct., vol. 199, 1998. 
[86] M. Bellare, "New Proofs for NMAC and HMAC: Security without Collision Resistance," J. Cryptol., 2015.

[87] L. Beringer, A. Petcher, K. Q. Ye, and A. W. Appel, "Verified correctness and security of OpenSSL HMAC [Complex!]," Usenix Sec, 2015.

[88] A. W. Appel, "Verification of a Cryptographic Primitive," ACM Trans. Program. Lang. Syst., 2015.

[89] Q. H. Dang, "FIPS 180-4 Secure Hash Standard,” 2015.

[90] X. Leroy, "Formal verification of a realistic compiler," Commun. ACM, vol. 52, no. 7, pp. 107-115, 2009.

[91] Y. Bertot and P. Castéran, Interactive Theorem Proving and Program Development: Coq'Art: The Calculus of Inductive Constructions. 2004.

[92] J. M. Turner, "The keyed-hash message authentication code (hmac)," Fed. Inf. Process. Stand. Publ., 2008.

[93] H. Krawczyk, R. Canetti, and M. Bellare, "HMAC: Keyed-hashing for message authentication," 1997.

[94] J.-K. Zinzindohoué, K. Bhargavan, J. Protzenko, and B. Beurdouche, "HACL*: A Verified Modern Cryptographic Library," CCS, 2017.

[95] D. J. Bernstein, T. Lange, and P. Schwabe, "The security impact of a new cryptographic library," in Lecture Notes in Computer Science (including subseries Lecture Notes in Artificial Intelligence and Lecture Notes in Bioinformatics), 2012.

[96] D. J. Bernstein, "Cryptography in NaCl," Netw. Cryptogr. Libr., vol. 3, p. 385, 2009.

[97] D. J. Bernstein, B. van Gastel, W. Janssen, T. Lange, P. Schwabe, and S. Smetsers, "TweetNaCl: A crypto library in 100 tweets," in Lecture Notes in Computer Science (including subseries Lecture Notes in Artificial Intelligence and Lecture Notes in Bioinformatics), 2015.

[98] G. Lowe, "Breaking and fixing the Needham-Schroeder public-key protocol using FDR," in Lecture Notes in Computer Science (including subseries Lecture Notes in Artificial Intelligence and Lecture Notes in Bioinformatics), 1996.

[99] J. Steiner, B. Neuman, and J. Schiller, "Kerberos: An Authentication Service for Open Network Systems.," USENIX Winter, 1988.

[100] K. Bhargavan, C. Fournet, M. Kohlweiss, A. Pironti, and P. Y. Strub, "Implementing TLS with Verified Cryptographic Security," in 2013 IEEE Symposium on Security and Privacy, 2013, pp. 445-459.

[101] L. Lamport, "The TLA Home Page," 2018. [Online]. Available: http://lamport.azurewebsites.net/tla/tla.html. [Accessed: 05-Jul-2018].

[102] C. Newcombe, "Why amazon chose TLA+," in International Conference on 
Abstract State Machines, Alloy, B, TLA, VDM, and Z, 2014, pp. 25-39.

[103] C. Newcombe, T. Rath, F. Zhang, B. Munteanu, M. Brooker, and M. Deardeuff, "Use of formal methods at Amazon Web Services," 2014.

[104] V. M. Igure, S. A. Laughter, and R. D. Williams, "Security issues in SCADA networks," Comput. Secur., vol. 25, no. 7, pp. 498-506, 2006.

[105] P. Huitsing, R. Chandia, M. Papa, and S. Shenoi, "Attack taxonomies for the Modbus protocols," Int. J. Crit. Infrastruct. Prot., 2008.

[106] S. East, J. Butts, M. Papa, and S. Shenoi, "A Taxonomy of Attacks on the DNP3 Protocol," in International Conference on Critical Infrastructure Protection, 2009, pp. 67-81.

[107] R. J. Lipton and L. Snyder, "A Linear Time Algorithm for Deciding Subject Security," J. ACM, 1977.

[108] M. Bishop and L. Snyder, "The Transfer of Information and Authority in a Protection System," in Proceedings of the Seventh ACM Symposium on Operating Systems Principles, 1979, pp. 45-54.

[109] T. Murray et al., "seL4: from general purpose to a proof of information flow enforcement," in Security and Privacy (SP), 2013 IEEE Symposium on, 2013, pp. 415-429.

[110] D. Elkaduwe, G. Klein, and K. Elphinstone, "Verified protection model of the seL4 microkernel," in Lecture Notes in Computer Science (including subseries Lecture Notes in Artificial Intelligence and Lecture Notes in Bioinformatics), 2008.

[111] L. C. Paulson, "Isabelle: The Next 700 Theorem Provers," in Logic and Computer Science, 1990.

[112] K. Fisher, J. Launchbury, and R. Richards, "The HACMS program: using formal methods to eliminate exploitable bugs," Phil. Trans. R. Soc. A, vol. 375, no. 2104, p. 20150401, 2017.

[113] "THREAT LANDSCAPE FOR INDUSTRIAL AUTOMATION SYSTEMS IN H2 2017," 2017. [Online]. Available: https://icscert.kaspersky.com/reports/2018/03/26/threat-landscape-for-industrial-automationsystems-in-h2-2017/. [Accessed: 04-Dec-2018].

[114] Kaspersky Lab ICS CERT, "THREAT LANDSCAPE FOR INDUSTRIAL AUTOMATION SYSTEMS IN THE SECOND HALF OF 2016.” Kaspersky Lab, 2017.

[115] M. Mimoso, "EQUATION APT GROUP ATTACK PLATFORM A STUDY IN STEALTH," threatpost, 2015. [Online]. Available: https://threatpost.com/equation-apt-group-attack-platform-a-study-instealth/111550/. [Accessed: 04-Dec-2018].

[116] M. Cheminod, A. Pironti, and R. Sisto, "Formal vulnerability analysis of a security 
system for remote fieldbus access," IEEE Trans. Ind. Informatics, 2011.

[117] B. Blanchet, "An Efficient Cryptographic Protocol Verifier Based on Prolog Rules," in 14th IEEE Computer Security Foundations Workshop (CSFW-14), 2001, pp. 82-96.

[118] M. Rocchetto and N. O. Tippenhauer, "Towards Formal Security Analysis of Industrial Control Systems," 2017.

[119] D. Dolev and A. C. Yao, "On the Security of Public Key Protocols," IEEE Trans. Inf. Theory, 1983.

[120] A. Jones, Z. Kong, and C. Belta, "Anomaly detection in cyber-physical systems: A formal methods approach," in Proceedings of the IEEE Conference on Decision and Control, 2014.

[121] "IEEE Standard for Electric Power Systems Communications-Distributed Network Protocol (DNP3) - Redline," IEEE Std 1815-2012 (Revision IEEE Std 1815-2010) - Redline, pp. 1-821, Oct. 2012.

[122] K. Jensen, "Coloured petri nets," in Lecture Notes in Computer Science (including subseries Lecture Notes in Artificial Intelligence and Lecture Notes in Bioinformatics), 1987.

[123] M. A. P. S. Modbus-IDA, "V. 1.1 b," Hopkinton, Massachusetts (www. modbus. org/docs/Modbus Appl. Proto col V1 1b. pdf), 2006.

[124] M. Sabraoui, J. L. Hieb, and J. H. Graham, "MODBUS protocol fuzzing for cybersecurity evaluation of industrial control systems," in 27th International Conference on Computer Applications in Industry and Engineering, CAINE 2014, 2014.

[125] J. Edmonds, M. Papa, and S. Shenoi, "Security analysis of multilayer SCADA protocols," in International Conference on Critical Infrastructure Protection, 2007, pp. 205-221.

[126] D. Potts, R. Bourquin, L. Andresen, J. Andronick, G. Klein, and G. Heiser, "Mathematically verified software kernels: raising the bar for high assurance implementations," 2014.

[127] E. Byres, "PLC Security Risk: Controller Operating Systems," Tofino Security Blog, 2011. [Online]. Available: https://www.tofinosecurity.com/blog/plc-securityrisk-controller-operating-systems.

[128] M. Sabraoui, "Sixnet Tools: for poking at Sixnet Things." DerbyCon, 2013.

[129] J. M. Rushby, Design and verification of secure systems, vol. 15, no. 5. ACM, 1981.

[130] J. Hieb, J. Graham, J. Schreiver, and K. Moss, "Security Preprocessor for Industrial Control Networks," in Proceedings of the 7th International Conference on Information Warfare and Security: ICIW, 2012, p. 130.

[131] L. Lamport, "Specifying Concurrent Systems with TLA^+," NATO ASI Ser. F 
Comput. Syst. Sci., vol. 173, pp. 183-250, 1999.

[132] J. Gray and L. Lamport, "Consensus on transaction commit," ACM Trans. Database Syst., vol. 31, no. 1, pp. 133-160, 2006.

[133] J. Andronick, "The formal verification of seL4," no. November, 2018.

[134] G. Klein, P. Derrin, and K. Elphinstone, "Experience report: seL4: formally verifying a high-performance microkernel," in ACM Sigplan Notices, 2009, vol. 44, no. 9, pp. 91-96.

[135] M. Fernandez, G. Klein, I. Kuz, and T. Murray, "CAmkES formalisation of a component platform," NICTA UNSW, 2013.

[136] I. Kuz, Y. Liu, I. Gorton, and G. Heiser, "CAmkES: A component model for secure microkernel-based embedded systems," J. Syst. Softw., vol. 80, no. 5, pp. 687-699, 2007.

[137] M. Fernandez, J. Andronick, G. Klein, and I. Kuz, "Automated verification of RPC stub code," in International Symposium on Formal Methods, 2015, pp. 273-290.

[138] S. Merz and H. Vanzetto, "Refinement Types for tla +," 2014.

[139] J. H. Hieb, J. H. Graham, and B. Luyster, "A Prototype Security Hardened Field Device for Industrial Control Systems," in Proceedings of the International Conference on Advanced Computing and Communications, 2010, pp. 95-100.

[140] M. Sabraoui, J. Hieb, A. Lauf, and J. H. Graham, "Using TLA+ to model and check bump-in-the-wire security for Industrial Control Systems," in Thirteenth IFIP WG 11.10 Conference on Critical Infrastructure Protection, 2019, pp. 167184.

[141] A. Helwer, "tla-experiments." Github, 2018.

[142] Y. Yu, P. Manolios, and L. Lamport, "Model checking TLA + specifications," in Lecture Notes in Computer Science (including subseries Lecture Notes in Artificial Intelligence and Lecture Notes in Bioinformatics), 1999.

[143] H. Krawczyk, M. Bellare, and R. Canetti, "HMAC: Keyed-Hashing for Message Authentication," 1997.

[144] L. Beringer, A. Petcher, K. Q. Ye, and A. W. Appel, "Verified correctness and security of OpenSSL HMAC," in Usenix Security Symposium, 2015.

[145] N. Swamy, J. Chen, C. Fournet, P.-Y. Strub, K. Bhargavan, and J. Yang, "Secure distributed programming with value-dependent types," in ACM SIGPLAN Notices, 2011, vol. 46, no. 9, pp. 266-278.

[146] Microsoft Research; INRIA, “FStarLang," 2018. [Online]. Available: https://www.fstar-lang.org/.

[147] K. Bhargavan et al., "Verified Low-Level Programming Embedded in F," arXiv Prepr. arXiv1703.00053, 2017. 


\section{APPENDICIES}

This appendix presents the ASCII table and TLA+ specification for the models used in chapter Chapter IV and Chapter V.

The ASCII Table.

\begin{tabular}{|c|c|c|c|c|c|c|c|c|c|c|c|c|c|c|c|c|c|c|}
\hline ec & $\mathrm{H}$ & 0 & h & & De & & & tml & hr & De & $\mathrm{H}$ & & III & & & & & \\
\hline 0 & 0 & 000 & NUL & (null) & 32 & & 040 & \&\#32； & & 64 & 40 & 100 & $\Leftrightarrow \# 64 ;$ & 96 & 60 & 140 & \&\#96; & \\
\hline 1 & 1 & 001 & $\mathrm{SOH}$ & (start of heading) & 33 & 21 & 041 & $\& \# 33 ;$ & $!$ & 65 & 41 & 101 & $\Leftrightarrow \# 65 ;$ is & 97 & 61 & 141 & \&\#97; & a \\
\hline 2 & 2 & 002 & STX & (start of text) & 34 & 22 & 042 & $\Leftrightarrow \# 34 ;$ & $r$ & 66 & 42 & 102 & $\Leftrightarrow \# 66 ; \mathrm{B}$ & 98 & 62 & 142 & \&\#98; & $\mathrm{b}$ \\
\hline 3 & 3 & 003 & ETX & (end of text) & 35 & 23 & 043 & & $\#$ & 67 & 43 & 103 & $\Leftrightarrow \# 67 ; C$ & 99 & 63 & 143 & 9; & c \\
\hline 4 & 4 & 004 & EOT & (end of transmission) & 36 & 24 & 044 & & $\xi$ & 68 & 44 & 104 & $\Leftrightarrow \# 68 ; \mathrm{D}$ & 100 & 64 & 144 & \&\#100; & d \\
\hline 5 & 5 & 005 & ENQ & (enquiry) & 37 & 25 & 045 & $\Leftrightarrow \# 37 ;$ & $\%$ & 69 & 45 & 105 & \&\#69; E & 101 & 65 & 145 & \&\#101; & e \\
\hline 6 & 6 & 006 & $\mathrm{ACK}$ & (acknowledge) & 38 & 26 & 046 & $\Leftrightarrow \# 38 ;$ & $\Leftrightarrow$ & 70 & 46 & 106 & $\Leftrightarrow \# 70 ; \mathrm{F}$ & 102 & 66 & 146 & 02; & $\mathrm{f}$ \\
\hline 7 & 7 & 007 & BEL & (bell) & 39 & 27 & 047 & $\Leftrightarrow \# 39 ;$ & 1 & 71 & 47 & 107 & $\Leftrightarrow \# 71 ; G$ & 103 & 67 & 147 & $03 ;$ & $g$ \\
\hline 8 & 8 & 010 & BS & (backspace) & 40 & 28 & 0.50 & $\Leftrightarrow \# 40 ;$ & l & 72 & 48 & 110 & $\Leftrightarrow \# 72 ; \mathrm{H}$ & 104 & 68 & 150 & 4 & $\mathrm{~h}$ \\
\hline 9 & 9 & 011 & TAB & (horizontal tab) & 41 & 29 & 051 & $\Leftrightarrow \# 41 ;$ & ) & 73 & 49 & 111 & $\Leftrightarrow \# 73 ; I$ & 105 & 69 & 151 & $05 ;$ & $i$ \\
\hline 10 & A & 012 & $\mathrm{LF}$ & (NL line feed, new line) & 42 & $2 \mathrm{~A}$ & 0.52 & $\Leftrightarrow \# 42 ;$ & $\pi$ & 74 & $4 \mathrm{~A}$ & 112 & $\Leftrightarrow \# 74 ; J$ & 106 & $6 \mathrm{~A}$ & 152 & 96; & j \\
\hline 11 & B & 013 & $\mathrm{VT}$ & (vertical tab) & 43 & $2 \mathrm{~B}$ & 0.53 & $\Leftrightarrow \# 43 ;$ & + & 75 & $4 \mathrm{~B}$ & 113 & $\Leftrightarrow \# 75 ; \mathrm{K}$ & 107 & $6 \mathrm{~B}$ & 153 & 07; & $\mathrm{k}$ \\
\hline 12 & $\mathrm{C}$ & 014 & FF & (NP form feed, new page & 44 & $2 \mathrm{C}$ & 054 & $\Leftrightarrow \# 44 ;$ & & 76 & $4 \mathrm{C}$ & 114 & $\Leftrightarrow \# 76 ; \mathrm{L}$ & 108 & $6 \mathrm{C}$ & 154 & 08; & 1 \\
\hline 13 & D & 015 & $\mathrm{CR}$ & (carriage return) & 45 & $2 \mathrm{D}$ & 055 & $\Leftrightarrow \# 45$ & - & 77 & $4 \mathrm{D}$ & 115 & $\Leftrightarrow \# 77 ; \mathrm{M}$ & 109 & $6 \mathrm{D}$ & 155 & 09 ; & In \\
\hline 14 & $\mathrm{E}$ & 016 & so & (shift out) & 46 & $2 \mathrm{E}$ & 0.56 & $\Leftrightarrow \# 46$; & . & 78 & $4 \mathrm{E}$ & 116 & $\Leftrightarrow \# 78 ; N$ & 110 & $6 \mathrm{E}$ & 156 & 0 ; & $\mathrm{n}$ \\
\hline 15 & $\mathrm{~F}$ & 017 & SI & (shift in) & 47 & $2 \mathrm{~F}$ & 057 & $\Leftrightarrow \# 47 ;$ & $/$ & 79 & $4 \mathrm{~F}$ & 117 & $\Leftrightarrow \# 79 ; 0$ & 111 & $6 \mathrm{~F}$ & 157 & $1 ;$ & 0 \\
\hline 16 & 10 & 020 & DLE & (data link escape) & 48 & 30 & 060 & \&\# 48; & 0 & 80 & 50 & 120 & $\Leftrightarrow \# 80 ; \mathrm{P}$ & 112 & 70 & 160 & $12 ;$ & $\mathrm{p}$ \\
\hline 17 & 11 & 021 & $\mathrm{DCl}$ & ce cont & 49 & 31 & 061 & $\Leftrightarrow \# 49 ;$ & 1 & 81 & 51 & 121 & $\Leftrightarrow \# 81 ; 0$ & 113 & 71 & 161 & 3 & $q$ \\
\hline 18 & 12 & 022 & $\mathrm{DC} 2$ & (device control 2) & 50 & 32 & 062 & $\Leftrightarrow \# 50 ;$ & 2 & 82 & 52 & 122 & $\Leftrightarrow \# 82 ; \mathrm{R}$ & 114 & 72 & 162 & 4 & r \\
\hline 19 & 13 & 023 & $\mathrm{DC} 3$ & (device control 3) & 51 & 33 & 063 & \&\#51; & 3 & 83 & 53 & 123 & \&\#83; 5 & 115 & 73 & 163 & 15 & $s$ \\
\hline 20 & 14 & 024 & $\mathrm{DC} 4$ & (device control 4) & 52 & 34 & 064 & $\Leftrightarrow \# 52 ;$ & 4 & 84 & 54 & 124 & $\Leftrightarrow \# 84 ; \mathrm{T}$ & 116 & 74 & 164 & 16; & $\mathrm{t}$ \\
\hline 21 & 15 & 025 & NAK & ative acknowledge) & 53 & 35 & 065 & $\Leftrightarrow \# 53 ;$ & 5 & 85 & 55 & 125 & $\Leftrightarrow \# 85 ; \mathrm{U}$ & 117 & 75 & 165 & 7 & $\mathrm{u}$ \\
\hline 22 & 16 & 026 & SYN & (synchronous idle) & 54 & 36 & 066 & $\Leftrightarrow \# 54 ;$ & 6 & 86 & 56 & 126 & $\Leftrightarrow \# 86 ; \mathrm{V}$ & 118 & 76 & 166 & 18; & $\mathrm{v}$ \\
\hline 23 & 17 & 027 & ETB & (end of trans. block & 55 & 37 & 067 & $\Leftrightarrow \# 55$ & 7 & 87 & 57 & 127 & $\Leftrightarrow \# 87 ;$ W & 119 & 77 & 167 & 19; & w \\
\hline 24 & 18 & 030 & CAN & (cancel) & 56 & 38 & 070 & $\Leftrightarrow \# 56$; & 8 & 88 & 58 & 130 & $\Leftrightarrow \# 88 ; \mathrm{X}$ & 120 & 78 & 170 & 20 & $\mathrm{x}$ \\
\hline 25 & 19 & 031 & EM & of mediu & 57 & 39 & 071 & $\Leftrightarrow \# 57$ & 9 & 89 & 59 & 131 & \&\#89; Y & 121 & 79 & 171 & $\Leftrightarrow \# 121 ;$ & $\mathrm{Y}$ \\
\hline 26 & lif & 032 & SUB & (substitute) & 58 & $3 \mathrm{~A}$ & 072 & $\Leftrightarrow \# 58 ;$ & : & 90 & $5 \mathrm{~A}$ & 132 & \&\#90; Z & 122 & $7 \mathrm{~A}$ & 172 & $\Leftrightarrow \# 122 ;$ & $z$ \\
\hline 27 & $1 \mathrm{~B}$ & 033 & ESC & (escape) & 59 & 3B & 073 & \&\#59; & ; & 91 & $5 \mathrm{~B}$ & 133 & \&\#91; [ & 123 & $7 \mathrm{~B}$ & 173 & \&\#123; & \{ \\
\hline 28 & $1 \mathrm{C}$ & 034 & FS & (file sep & 60 & $3 \mathrm{C}$ & 074 & $\& \# 60 ;$ & $<$ & 92 & $5 \mathrm{C}$ & 134 & $\Leftrightarrow \# 92 ;$ & 124 & $7 \mathrm{C}$ & 174 & 24; & I \\
\hline 29 & 1D & 035 & GS & גp separator) & 61 & $3 \mathrm{D}$ & 075 & $\Leftrightarrow \# 61 ;$ & $=$ & 93 & $5 \mathrm{D}$ & 135 & \&\#93; ] & 125 & $7 \mathrm{D}$ & 175 & \&\#125; & \} \\
\hline 30 & $1 \mathrm{E}$ & 036 & RS & (record separator) & 62 & $3 \mathrm{E}$ & 076 & $\Leftrightarrow \# 62 ;$ & & 94 & $5 \mathrm{E}$ & 136 & \&\#94; ᄉ & 126 & $7 \mathrm{E}$ & 176 & \&\#126; & $\sim$ \\
\hline 31 & $1 \mathrm{~F}$ & 037 & US & (unit separator) & 63 & $3 \mathrm{~F}$ & 077 & $\Leftrightarrow \# 63$ & $?$ & 95 & $5 \mathrm{~F}$ & 137 & $\Leftrightarrow \# 95 ;$ & 127 & $7 \mathrm{~F}$ & 177 & \&\#127; & \\
\hline
\end{tabular}

Source: www.LookupTables.coin

For TLA+ and CAmkES code, as well as the seL4 prototype for the security preprocessor, see https://github.com/mssabr01/Dissertation-Work 


\section{CURRICULUM VITA}

NAME: $\quad$ Mehdi Sabraoui

ADDRESS: $\quad$ Department of Computer Science and Engineering 222 Eastern Pkwy.

University of Louisville

Louisville, KY 40208

DOB: $\quad$ Louisville, Kentucky - February 19, 1990

EDUCATION

\& TRAINING: B.S., Computer Engineering and Computer Science

University of Louisville

2008-2013

M.Eng., Computer Engineering and Computer Science

University of Louisville

2012-2013

Ph.D., Computer Science and Engineering

University of Louisville

2014-2019

PUBLICATIONS:

Using TLA+ to model and check bump-in-the-wire security for

Industrial Control Systems Thirteenth IFIP

WG 11.10 Conference on Critical Infrastructure Protection

2019

Verifying SCADA Security in Industrial Control Systems 29th International Conference on Computer Applications in Industry and Engineering 2016

MODBUS protocol fuzzing for cyber-security evaluation of industrial control systems 27th International Conference on Computer Applications in Industry and Engineering 2014 